\title{
Strong Feller Property for SDEs Driven by Multiplicative Cylindrical Stable Noise
}

\section{Tadeusz Kulczycki $^{1}$ • Michał Ryznar ${ }^{1}$ (D) . Paweł Sztonyk ${ }^{1}$}

Received: 20 December 2018 / Accepted: 5 May 2020 / Published online: 15 June 2020

(C) The Author(s) 2020

\begin{abstract}
We consider the stochastic differential equation $d X_{t}=A\left(X_{t-}\right) d Z_{t}, X_{0}=x$, driven by cylindrical $\alpha$-stable process $Z_{t}$ in $\mathbb{R}^{d}$, where $\alpha \in(0,1)$ and $d \geq 2$. We assume that the determinant of $A(x)=\left(a_{i j}(x)\right)$ is bounded away from zero, and $a_{i j}(x)$ are bounded and Lipschitz continuous. We show that for any fixed $\gamma \in(0, \alpha)$ the semigroup $P_{t}$ of the process $X_{t}$ satisfies $\left|P_{t} f(x)-P_{t} f(y)\right| \leq c t^{-\gamma / \alpha}|x-y|^{\gamma}\|f\|_{\infty}$ for arbitrary bounded Borel function $f$. Our approach is based on Levi's method.
\end{abstract}

Keywords Strong Feller property - Stochastic differential equations - Cylindrical stable processes $\cdot$ Semigroups of operators

Mathematics Subject Classification (2010) 60H10 · 60G52

\section{Introduction}

Let $Z_{t}=\left(Z_{t}^{(1)}, \ldots, Z_{t}^{(d)}\right)^{T}$ be a cylindrical $\alpha$-stable process, that is $Z_{t}^{(1)}, \ldots, Z_{t}^{(d)}$ are independent one-dimensional symmetric standard $\alpha$-stable processes of index $\alpha \in(0,1)$, $d \in \mathbb{N}, d \geq 2$. We consider the stochastic differential equations

$$
d X_{t}=A\left(X_{t-}\right) d Z_{t}, \quad X_{0}=x \in \mathbb{R}^{d},
$$

T. Kulczycki was supported in part by the National Science Centre, Poland, grant no. 2015/17/B/ST1/01233, M. Ryznar was supported in part by the National Science Centre, Poland, grant no. 2015/17/B/ST1/01043, P. Sztonyk was supported in part by the National Science Centre, Poland, grant no. 2017/27/B/ST1/01339. This work was supported in part by Wrocław University of Science and Technology grant 049U/0052/19.

Michał Ryznar

Michal.Ryznar@pwr.edu.pl

Tadeusz Kulczycki

Tadeusz.Kulczycki@pwr.edu.pl

Paweł Sztonyk

Pawel.Sztonyk@pwr.edu.pl

1 Faculty of Pure and Applied Mathematics, Wrocław University of Science and Technology, Wyb. Wyspiańskiego 27, 50-370, Wrocław, Poland 
where $A(x)=\left(a_{i j}(x)\right)$ is a $d \times d$ matrix and there are constants $\eta_{1}, \eta_{2}, \eta_{3}>0$, such that for any $x, y \in \mathbb{R}^{d}, i, j \in\{1, \ldots, d\}$,

$$
\begin{gathered}
\left|a_{i j}(x)\right| \leq \eta_{1}, \\
\operatorname{det}(A(x)) \geq \eta_{2}, \\
\left|a_{i j}(x)-a_{i j}(y)\right| \leq \eta_{3}|x-y| .
\end{gathered}
$$

It is well known that the SDE (1) has a unique strong solution $X_{t}$, see e.g. [30, Theorem 34.7 and Corollary 35.3]. By [33, Corollary 3.3], $X_{t}$ is a Feller process.

Let $\mathbb{E}^{x}$ denote the expected value of the process $X$ starting from $x$ and $\mathcal{B}_{b}\left(\mathbb{R}^{d}\right)$ denote the set of all Borel bounded functions $f: \mathbb{R}^{d} \rightarrow \mathbb{R}$. For any $t \geq 0, x \in \mathbb{R}^{d}$ and $f \in \mathcal{B}_{b}\left(\mathbb{R}^{d}\right)$ we put

$$
P_{t} f(x)=\mathbb{E}^{x} f\left(X_{t}\right) .
$$

The main result of this paper is the following theorem.

Theorem 1.1 For any $\gamma \in(0, \alpha), \tau>0, t \in(0, \tau], x, y \in \mathbb{R}^{d}$ and $f \in \mathcal{B}_{b}\left(\mathbb{R}^{d}\right)$ we have

$$
\left|P_{t} f(x)-P_{t} f(y)\right| \leq c t^{-\gamma / \alpha}|x-y|^{\gamma}\|f\|_{\infty},
$$

where $c$ depends on $\tau, \alpha, d, \eta_{1}, \eta_{2}, \eta_{3}, \gamma$.

Recently, estimates of the type $\left|P_{t} f(x)-P_{t} f(y)\right| \leq c_{t}|x-y|^{\gamma}\|f\|_{\infty}$ or $\left|\nabla_{x} P_{t} f(x)\right| \leq$ $c_{t}\|f\|_{\infty}$ have been intensively studied for semigroups of solutions of SDEs $d X_{t}=$ $A\left(X_{t-}\right) d Z_{t}+b\left(X_{t}\right) d t$ driven by Lévy processes $Z$ with jumps. In [37] such results were proved for the Orstein-Uhlenbeck jump process $X$. In [38] the above estimates were shown in the case when the driving process $Z$ is a subordinated Brownian motion. There are also known results when the Lévy measure $v$ of $Z$ satisfies $v(d z) \geq c 1_{|z| \leq r}|z|^{-d-\alpha} d z$ for some $\alpha \in(0,2)$ and $c, r>0$, see [27]. Another well explored case is when $Z$ has a nondegenerate diffusion part, as done in [40]. The above estimates were also studied in [32] for solutions of SDEs in infinite dimensional Hilbert space driven by an additive cylindrical stable noise (see [32, Theorem 5.7]).

Note that Theorem 1.1 implies the strong Feller property of the semigroup $P_{t}$. This property also follows from [10], see Remark 4.23. The strong Feller property for SDEs driven by additive cylindrical Lévy processes have been intensively studied recently (see e.g. [11, $32,39])$. It is worth mentioning that the strong Feller property and gradient estimates for the semigroups associated to SDEs driven by Lévy processes in $\mathbb{R}^{d}$ with jumps, with absolutely continuous Lévy measures, have been examined for many years (see e.g. [25, 28, 34, $36,38,40,42])$.

The SDE (1) (with multiplicative noise) was studied by Bass and Chen in [1]. They proved existence and uniqueness of weak solutions of the SDE (1) under very mild assumptions on matrices $A(x)$ (i.e. they assumed that $A(x)$ are continuous and bounded in $x$ and nondegenerate for each $x$ ). In [24] the SDE (1) was considered for diagonal matrices $A(x)$ whose diagonal coefficients are bounded away from zero, from infinity and Hölder continuous. Under these assumptions the corresponding transition density $p^{A}(t, x, y)$ was constructed and Hölder estimates $x \rightarrow p^{A}(t, x, y)$ were obtained. These estimates imply the strong Feller property of the corresponding semigroup.

The case of non-diagonal matrices $A(x)$, treated in this paper, is much more difficult. We use Levi's method to construct the semigroup $P_{t}$ and to prove Theorem 1.1. However, there are many problems in applying this method to the case when multiplicative coefficient $A(x)$ in the SDE (1) may perform a non-trivial rotation. Therefore we had to introduce some new ideas. Below we briefly describe the main steps in our approach. 
Let us put

$$
\mathcal{K} f(x)=\mathcal{A}_{\alpha} \sum_{i=1}^{d} \lim _{\zeta \rightarrow 0^{+}} \int_{|w|>\zeta}\left[f\left(x+a_{i}(x) w\right)-f(x)\right] \frac{d w}{|w|^{1+\alpha}},
$$

where $a_{i}(x)=\left(a_{1 i}(x), \ldots, a_{d i}(x)\right)$ and $\mathcal{A}_{\alpha}=2^{\alpha} \Gamma((1+\alpha) / 2) /\left(\pi^{1 / 2}|\Gamma(-\alpha / 2)|\right)$. It is well known (see e.g. ([1, Proposition 4.1]) that $\mathcal{K}$ is the generator of the process $X$.

We divide $\mathcal{K}$ into two parts

$$
\mathcal{K} f(x)=\mathcal{L} f(x)+\mathcal{R} f(x),
$$

where

$$
\mathcal{L} f(x)=\sum_{i=1}^{d} \lim _{\zeta \rightarrow 0^{+}} \int_{|w|>\zeta}\left[f\left(x+a_{i}(x) w\right)-f(x)\right] \mu(w) d w,
$$

and $\mu(w) d w$ is the appropriate truncation of the Lévy measure $|w|^{-1-\alpha} d w(\operatorname{supp}(\mu) \subset$ $[-1,1])$. The definition of the truncated measure is presented at the beginning of Section 2. Roughly speaking, $\mathcal{L}$ corresponds to small jumps of the process $X$ and $\mathcal{R}$ corresponds to big jumps of the process $X$. Our first aim is to construct the heat kernel $u(t, x, y)$ corresponding to the operator $\mathcal{L}$. This is done by using Levi's method, which is heuristically explained below.

At first we choose a kernel $\tilde{u}(t, x, y)$ which serves as the first order approximation of $u(t, x, y)$. By $r(t, x, y)$ we denote the resulting error, that is $u(t, x, y)=\tilde{u}(t, x, y)+$ $r(t, x, y)$. Put

$$
q_{0}(t, x, y)=\left(\frac{\partial}{\partial t}-\mathcal{L}\right) r(t, \cdot, y)(x)=-\left(\frac{\partial}{\partial t}-\mathcal{L}\right) \tilde{u}(t, \cdot, y)(x) .
$$

Since $u(t, x, y)$ is the heat kernel for $\mathcal{L}$, at least formally, one has

$$
r(t, x, y)=\int_{0}^{t} \int_{\mathbb{R}^{d}} u(t-s, x, z) q_{0}(s, z, y) d z d s,
$$

so

$$
u(t, x, y)=\tilde{u}(t, x, y)+\int_{0}^{t} \int_{\mathbb{R}^{d}} u(t-s, x, z) q_{0}(s, z, y) d z d s .
$$

The formal solution of this equation is given by the formula

$$
u(t, x, y)=\tilde{u}(t, x, y)+\sum_{n=0}^{\infty} \int_{0}^{t} \int_{\mathbb{R}^{d}} \tilde{u}(t-s, x, z) q_{n}(s, z, y) d z d s,
$$

where $q_{n}$ is defined inductively as

$$
q_{n}(t, x, y)=\int_{0}^{t} \int_{\mathbb{R}^{d}} q_{0}(t-s, x, z) q_{n-1}(s, z, y) d z d s .
$$

As the approximation $\tilde{u}(t, x, y)$ we choose the heat kernel of the operator $\mathcal{L}$ frozen at $y \in \mathbb{R}^{d}$. More precisely, we put $\tilde{u}(t, x, y)=p_{y}(t, x-y)$, where $p_{y}(t, \cdot)$ is the heat kernel of $\mathcal{L}^{y}$ (i.e. $\frac{\partial}{\partial t} p_{y}(t, z)=\mathcal{L}^{y} p_{y}(t, \cdot)(z), t>0, z \in \mathbb{R}^{d}$ ), where

$$
\mathcal{L}^{y} f(x)=\mathcal{A}_{\alpha} \sum_{i=1}^{d} \lim _{\zeta \rightarrow 0^{+}} \int_{|w|>\zeta}\left[f\left(x+a_{i}(y) w\right)-f(x)\right] \mu(w) d w .
$$

Typically, in many papers using Levi's method, the first step was to obtain precise bounds for $q_{0}(t, x, y)$ which allow to estimate $q_{n}(t, x, y)$ inductively point-wise. In our case it seems impossible to obtain such precise bounds, hence we prove (see Proposition 3.9) some 
crude estimates for $q_{0}(t, x, y)$ and its integrals with respect to $d x$ or $d y$, which are sufficient for our purposes. The main tools to prove Proposition 3.9 are Lemma 3.6 and the estimate (18). This key estimate (18) is proven using the techniques and results from [23], [22] and [35]. After constructing the transition density $u(t, x, y)$ we use the technique developed by Knopova and Kulik [19] to show that $U_{t} f(x):=\int_{\mathbb{R}^{d}} u(t, x, y) f(y) d y$ satisfies the appropriate heat equation in the so-called approximate setting. In the next step we construct the semigroup $T_{t}$ for the solution of the SDE (1) (driven by the not truncated process). Roughly speaking, this construction is based on adding big jumps to the truncated process (Meyer-type construction). Next we show that $T_{t} f(x)$ satisfies the appropriate heat equation in the approximate setting (see Lemma 4.18), which allows to prove that the constructed semigroup $T_{t}$ is in fact the semigroup $P_{t}$.

Let us introduce the operator $\mathcal{K}$ frozen at a point $y \in \mathbb{R}^{d}$,

$$
\mathcal{K}^{y} f(x)=\mathcal{A}_{\alpha} \sum_{i=1}^{d} \lim _{\zeta \rightarrow 0^{+}} \int_{|w|>\zeta}\left[f\left(x+a_{i}(y) w\right)-f(x)\right] \frac{d w}{|w|^{1+\alpha}} .
$$

Let $p_{y}^{\mathcal{K}}(t, \cdot)$ be the heat kernel of $\mathcal{K}^{y}$, that is $\frac{\partial}{\partial t} p_{y}^{\mathcal{K}}(t, z)=\mathcal{K}^{y} p_{y}^{\mathcal{K}}(t, \cdot)(z)$ for any $t>0$, $z \in \mathbb{R}^{d}$. One may ask why the Levi method is applied to the operator $\mathcal{L}$ (corresponding to the truncated Lévy measure) and not to the operator $\mathcal{K}$. The reason is that (in general) the heat kernel $p_{y}^{\mathcal{K}}(t, \cdot)$ of the operator $\mathcal{K}^{y}$ does not have good integrability properties, i.e. $\int_{\mathbb{R}^{d}} p_{y}^{\mathcal{K}}(t, x-y) d y=\infty$ for some choices of matrices $A(x)$ and some $t>0, x \in \mathbb{R}^{d}$. Therefore it cannot be used as the first order approximation of the heat kernel of $\mathcal{K}$.

Our current technique is restricted to the case $\alpha \in(0,1)$. The main difficulty for $\alpha \in$ $[1,2)$ is that in such case one has to effectively estimate the expression

$$
p_{y}\left(t, x+a_{i}(x) w\right)+p_{y}\left(t, x-a_{i}(x) w\right)-2 p_{y}(t, x)
$$

instead of

$$
p_{y}\left(t, x+a_{i}(x) w\right)-p_{y}(t, x),
$$

where $p_{y}(t, x)$ is the frozen density for the truncated process (see Section 3 for the precise definition of $\left.p_{y}(t, x)\right)$. Our crucial estimate (18) allows a suitable estimate of (13) but fails to bound (12) in a way sufficient for our purpose.

We point out that the existence of transition densities $p(t, x, y)$ of the process $X$ is already well known, see [10]. In our paper we obtain a representation of this densities, see Remark 4.26. One may ask about the boundedness of $p(t, x, y)$. It turns out that for some choices of matrices $A(x)$ (satisfying (2), (3), (4)) and for some $t>0, x \in \mathbb{R}^{d}$ we have $p(t, x, \cdot) \notin L^{\infty}\left(\mathbb{R}^{d}\right)$, see Remarks 4.24 and 4.25. Nevertheless we have the following regularity result.

Theorem 1.2 For any $\gamma \in(0, \alpha / d), \tau>0, t \in(0, \tau], x \in \mathbb{R}^{d}$ and $f \in L^{1}\left(\mathbb{R}^{d}\right) \cap L^{\infty}\left(\mathbb{R}^{d}\right)$ we have

$$
\left|P_{t} f(x)\right| \leq c t^{-\gamma d / \alpha}\|f\|_{\infty}^{1-\gamma}\|f\|_{1}^{\gamma},
$$

where $c$ depends on $\tau, \alpha, d, \eta_{1}, \eta_{2}, \eta_{3}, \gamma$.

The paper is organized as follows. In Section 2 we study properties of the transition density of a suitably truncated one-dimensional stable process. These properties are crucial in the sequel. In Section 3 we construct the transition density $u(t, x, y)$ of the solution of (1) driven by the truncated process. We also show that it satisfies the appropriate equation in the approximate setting. In Section 4 we construct the transition semigroup of the solution of (1). We also prove Theorems 1.1 and 1.2. 


\section{Preliminaries}

All constants appearing in this paper are positive and finite. In the whole paper we fix $\tau>0$, $\alpha \in(0,1), d \in \mathbb{N}, d \geq 2, \eta_{1}, \eta_{2}, \eta_{3}$, where $\eta_{1}, \eta_{2}, \eta_{3}$ appear in (2), (3) and (4). We adopt the convention that constants denoted by $c$ (or $c_{1}, c_{2}, \ldots$ ) may change their value from one use to the next. In the whole paper, unless is explicitly stated otherwise, we understand that constants denoted by $c$ (or $c_{1}, c_{2}, \ldots$ ) depend on $\tau, \alpha, d, \eta_{1}, \eta_{2}, \eta_{3}$. We also understand that they may depend on the choice of the constants $\varepsilon$ and $\gamma$. We write $f(x) \approx g(x)$ for $x \in A$ if $f, g \geq 0$ on $A$ and there is a constant $c \geq 1$ such that $c^{-1} f(x) \leq g(x) \leq c f(x)$ for $x \in A$. The standard inner product for $x, y \in \mathbb{R}^{\bar{d}}$ we denote by $x \cdot y$.

For any $t>0, x \in \mathbb{R}^{d}$ we define the measure $\sigma_{t}(x, \cdot)$ by

$$
\sigma_{t}(x, A)=\mathbb{P}^{x}\left(X_{t} \in A\right),
$$

for any Borel set $A \subset \mathbb{R}^{d} . \mathbb{P}^{x}$ denotes the distribution of the process $X$ starting from $x \in \mathbb{R}^{d}$. For any $t>0, x \in \mathbb{R}^{d}$ we have

$$
P_{t} f(x)=\int_{\mathbb{R}^{d}} f(y) \sigma_{t}(x, d y), \quad f \in \mathcal{B}_{b}\left(\mathbb{R}^{d}\right) .
$$

It is well known that the density of the Lévy measure of the one-dimensional symmetric standard $\alpha$-stable process is given by $\mathcal{A}_{\alpha}|x|^{-1-\alpha}$. In the sequel we will need to truncate this density. The truncated density will be denoted by $\mu^{(\delta)}(x)$. For $\delta \in(0,1]$ we would like to construct a function $\mu^{(\delta)}: \mathbb{R} \backslash\{0\} \rightarrow[0, \infty)$ such that for $x \in(0, \delta]$ we have $\mu^{(\delta)}(x)=\mathcal{A}_{\alpha}|x|^{-1-\alpha}$, for $x \in(\delta, 2 \delta)$ we have $\mu^{(\delta)}(x) \in\left(0, \mathcal{A}_{\alpha}|x|^{-1-\alpha}\right)$ and $\mu^{(\delta)}(x)=0$ for $x \geq 2 \delta$. Moreover, $\mu^{(\delta)}$ is defined so that it is nonincreasing, convex and $C^{1}$ on $(0, \infty)$ and satisfies $\mu^{(\delta)}(-x)=\mu^{(\delta)}(x)$ for $x \in(0, \infty)$.

At first we find $\mu^{(\delta)}$ for $\delta=1$. Let $\nu(x)=\mathcal{A}_{\alpha}|x|^{-1-\alpha}$. Observe that the tangent line to the graph of $v(x)$ at $(1, v(1))$ is $l: y-\mathcal{A}_{\alpha}=-\mathcal{A}_{\alpha}(1+\alpha)(x-1)$ and it crosses the horizontal axes at $x_{0}=1+\frac{1}{1+\alpha}<2$. Let $(x-2)^{2}+(y-r)^{2}=r^{2}, r>0$, be a circle tangent to the horizontal axes at the point $(2,0)$ and tangent to the line $l$. Let $(\tilde{x}, \tilde{y})$ be the point at which the circle touches the line $l$. It is clear that $1<\tilde{x}<x_{0}$. We put

$$
\mu^{(1)}(x)= \begin{cases}v(x) & \text { for } 0<|x| \leq 1, \\ \mathcal{A}_{\alpha}-\mathcal{A}_{\alpha}(1+\alpha)(|x|-1) & \text { for } 1<|x| \leq \tilde{x} \\ r-\sqrt{r^{2}-(|x|-2)^{2}} & \text { for } \tilde{x}<|x| \leq 2 \\ 0 & \text { for }|x|>2\end{cases}
$$

It is obvious that $\mu^{(1)}$ is convex, decreasing, continuously differentiable on $(0, \infty)$ and satisfies all the other requirements if $\delta=1$. For arbitrary $\delta>0$ we define

$$
\mu^{(\delta)}(x)=\delta^{-\alpha-1} \mu^{(1)}(x / \delta) .
$$

Since $v(x)=\delta^{-\alpha-1} v(x / \delta)$ we see that $\mu^{(\delta)}$ possess all desired properties since $\mu^{(1)}$ does.

We also define

$$
\mathcal{G}^{(\delta)} f(x)=\lim _{\zeta \rightarrow 0^{+}} \int_{|w|>\zeta}(f(x+w)-f(x)) \mu^{(\delta)}(w) d w .
$$

By $g_{t}^{(\delta)}$ we denote the heat kernel corresponding to $\mathcal{G}^{(\delta)}$ that is

$$
\begin{gathered}
\frac{\partial}{\partial t} g_{t}^{(\delta)}(x)=\mathcal{G}^{(\delta)} g_{t}^{(\delta)}(x), \quad t>0, x \in \mathbb{R}, \\
\int_{\mathbb{R}} g_{t}^{(\delta)}(x) d x=1, \quad t>0 .
\end{gathered}
$$


It is well known that $g_{t}^{(\delta)}$ belongs to $C^{1}((0, \infty))$ as a function of $t$ and belongs to $C^{2}(\mathbb{R})$ as a function of $z$. We also note that as a function of $x$ it is symmetric and nonincreasing on $[0, \infty)$. This is due to the fact that $\mu^{(\delta)}$ also has that property.

For any $\varepsilon \in(0,1], \tau>0, t \in(0, \infty)$ and $x \in \mathbb{R}$ we define

$$
h_{t}^{(\varepsilon)}(x)= \begin{cases}\frac{t}{\left(|x|+t^{1 / \alpha}\right)^{1+\alpha}} & \text { for }|x|<\varepsilon \\ c_{\varepsilon} t^{1+(d-1) / \alpha} e^{-|x|} & \text { for }|x| \geq \varepsilon\end{cases}
$$

where $c_{\varepsilon}=\frac{e^{\varepsilon}}{\left(1+\tau^{1 / \alpha}\right)^{1+\alpha} \tau^{(d-1) / \alpha}}$. The constant $c_{\varepsilon}$ is chosen so that for any $t \in(0, \tau]$ the function $x \rightarrow h_{t}^{(\varepsilon)}(x)$ is nonincreasing on $[0, \infty)$.

Lemma 2.1 For any $\varepsilon \in(0,1]$, there exists $c$ such that for $\delta=\varepsilon \min \left\{\frac{\alpha}{8(\alpha+d+2)}, \frac{1}{4 d\left(\eta_{1} \vee 1\right)^{2}}\right\}$, and any $t \in(0, \tau], x, y \in \mathbb{R}$, we have

$$
\begin{aligned}
g_{t}^{(\delta)}(x) & \leq c h_{t}^{(\varepsilon)}(|x|) \\
\left|g_{t}^{(\delta)}(x)-g_{t}^{(\delta)}(y)\right| & \leq c|x-y|\left(\frac{h_{t}^{(\varepsilon)}(|x|)}{t^{1 / \alpha}+|x|}+\frac{h_{t}^{(\varepsilon)}(|y|)}{t^{1 / \alpha}+|y|}\right) .
\end{aligned}
$$

Proof Our proof, specially of (18), will relay on the relationship between transition densities of isotropic and unimodal Lévy processes living in spaces of different dimensions. Namely, under certain conditions, the gradient of the transition density of one process living in $\mathbb{R}^{n}$ can be expressed in terms of the transition density of the other process living in $\mathbb{R}^{n+2}$, see Theorem 1.5 in [23].

Hence, at first we consider a general case of heat kernels $g^{(\delta, n)}$ on $\mathbb{R}^{n}$ for $\delta \in(0,1]$, $n \in\{1,2, \ldots\}$ such that

$$
g_{t}^{(\delta, n)}(x)=\frac{1}{(2 \pi)^{n}} \int_{\mathbb{R}^{n}} e^{-i x \cdot u} e^{-t \Phi_{\delta}^{(n)}(u)} d u,
$$

where

$$
\Phi_{\delta}^{(n)}(u)=\int_{\mathbb{R}^{n}}(1-\cos (u \cdot y)) \mu^{(\delta, n)}(y) d y, \quad u \in \mathbb{R}^{n},
$$

and $\mu^{(\delta, n)}(y)=\mu^{(\delta, n)}(|y|)$ is isotropic unimodal Lévy density such that $\mu^{(\delta, n)}(y) \approx$ $|y|^{-n-\alpha}$ for $|y| \leq \delta$, and $\mu^{(\delta, n)}(y)=0$ for $|y| \geq 2 \delta$. In the proof of this lemma we assume that constants $c$ may additionally depend on $n$. It follows from Lemma 1 in [3] that

$$
\frac{2}{n \pi^{2}} H(|u|) \leq \Phi_{\delta}^{(n)}(u) \leq 2 H(|u|), \quad u \in \mathbb{R}^{n}
$$

where

$$
H(r)=\int_{\mathbb{R}^{n}}\left(1 \wedge(r|y|)^{2}\right) \mu^{(\delta, n)}(y) d y, \quad r \geq 0,
$$

hence we easily obtain

$$
\Phi_{\delta}^{(n)}(u) \geq c|u|^{2} \int_{0}^{\frac{1}{|u|} \wedge \delta} r^{1-\alpha} d r=c \frac{1}{\delta^{\alpha}}\left((\delta|u|)^{2} \wedge(\delta|u|)^{\alpha}\right), \quad u \in \mathbb{R}^{n} .
$$

Similarly,

$$
\Phi_{\delta}^{(n)}(u) \leq c \frac{1}{\delta^{\alpha}}\left((\delta|u|)^{2} \wedge(\delta|u|)^{\alpha}\right), \quad u \in \mathbb{R}^{n}
$$


In particular the symbol $\Phi_{\delta}^{(n)}$ has global weak lower scaling property with index $\alpha$ (see [3]). This yields, by Theorem 21 of [3],

$$
g_{t}^{(\delta, n)}(x) \leq c \min \left\{\left(H^{-1}(1 / t)\right)^{n}, t H(1 /|x|)|x|^{-n}\right\}, \quad t>0, x \in \mathbb{R}^{n} .
$$

Observing that $H(r) \leq c r^{\alpha}$ for $r \geq 0$ and $H^{-1}(1 / t) \leq c \frac{1}{t^{1 / \alpha}}$, for $t \leq \tau$, we have

$$
g_{t}^{(\delta, n)}(x) \leq c \frac{t}{\left(|x|+t^{1 / \alpha}\right)^{n+\alpha}}, \quad x \in \mathbb{R}^{n}, t \in(0, \tau] .
$$

Let $t \leq 1 \wedge \tau$. Using Lemma 4.2 from [35] we get

$$
\begin{aligned}
g_{t}^{(\delta, n)}(x) & \leq e^{\frac{-|x|}{8 \delta} \log \left(\frac{\delta|x|}{t m_{0}}\right)} g_{t}^{(\delta, n)}(0)=\left(\frac{t m_{0}}{\delta|x|}\right)^{\frac{|x|}{8 \delta}} g_{t}^{(\delta, n)}(0) \\
& \leq c t^{\frac{|x|}{8 \delta}-\frac{n}{\alpha}} e^{\frac{-|x|}{8 \delta} \log \left(\frac{\delta|x|}{m_{0}}\right)}, \quad|x| \geq \frac{e m_{0}}{\delta} t,
\end{aligned}
$$

where $m_{0}=m_{0}(\delta, n)=\int_{\mathbb{R}^{n}}|y|^{2} \mu^{(\delta, n)}(y) d y<\infty$. This yields

$$
g_{t}^{(\delta, n)}(x) \leq c t^{1+\frac{d-1}{\alpha}} e^{\frac{-|x|}{8 \delta} \log \left(\frac{\delta|x|}{m_{0}}\right)},
$$

provided $|x| \geq \max \left\{8 \delta\left(1+\frac{n+d-1}{\alpha}\right), \frac{e m_{0}}{\delta} t\right\}$. We observe that there exists $c_{1}=c_{1}(\delta, \alpha, d, n)$ such that

$$
e^{\frac{-|x|}{8 \delta} \log \left(\frac{\delta|x|}{m_{0}}\right)} \leq c_{1} e^{-(\alpha+d)|x| / \alpha}, \quad x \in \mathbb{R}^{n},
$$

so we obtain

$$
g_{t}^{(\delta, n)}(x) \leq c_{2} t^{1+\frac{d-1}{\alpha}} e^{-(\alpha+d)|x| / \alpha}, \quad|x| \geq \max \left\{8 \delta\left(1+\frac{n+d-1}{\alpha}\right), \frac{e m_{0}}{\delta} t\right\},
$$

with $c_{2}=c_{2}(\delta, \alpha, d, n)$.

Let $1 \leq t \leq \tau$. Using again Lemma 4.2 from [35] we get

$$
\begin{aligned}
g_{t}^{(\delta, n)}(x) & \leq e^{\frac{-|x|}{8 \delta} \log \left(\frac{\delta|x|}{t m_{0}}\right)} g_{t}^{(\delta, n)}(0) \leq e^{\frac{-|x|}{8 \delta}} g_{1}^{(\delta, n)}(0) \\
& \leq c t^{1+\frac{d-1}{\alpha}} e^{\frac{-|x|}{8 \delta}}, \quad|x| \geq \frac{e m_{0}}{\delta} t .
\end{aligned}
$$

Observe that with $\delta=\varepsilon \min \left\{\frac{\alpha}{8(\alpha+d+2)}, \frac{1}{4 d\left(\eta_{1} \vee 1\right)^{2}}\right\}$ the estimate (17) follows from Eq. 19, Eq. 20 and Eq. 21 with $n=1$.

The function $\mu^{(\delta, 1)}=\mu^{(\delta)}$ satisfies the assumption of Theorem 1.5 in [23] which yields that there exists a Lévy process $X_{t}^{(3)}$ in $\mathbb{R}^{3}$ with the characteristic exponent $\Phi_{\delta}^{(3)}(u)=$ $\Phi_{\delta}^{(3)}(|u|), u \in \mathbb{R}^{3}$ and the radial, radially nonincreasing transition density $g_{t}^{(\delta, 3)}(x)=$ $g_{t}^{(\delta, 3)}(|x|)$ satisfying

$$
g_{t}^{(\delta, 3)}(r)=\frac{-1}{2 \pi r} \frac{d}{d r} g_{t}^{(\delta, 1)}(r), \quad r>0 .
$$

Furthermore it follows from the proof of Theorem 1.5 in [23] that the Lévy measure of the process $X_{t}^{(3)}$ is given by $v^{(\delta, 3)}(d x)=\mu^{(\delta, 3)}(|x|) d x$, where $\mu^{(\delta, 3)}(r)=$ $\frac{-1}{2 \pi r} \frac{d \mu^{(\delta, 1)}}{d r}(r), r>0$. In particular, $\mu^{(\delta, 3)}(r)$ is nonincreasing on $(0, \infty)$ and we have $\mu^{(\delta, 3)}(x)=\frac{\mathcal{A}_{\alpha}}{2 \pi(2+\alpha)}|x|^{-3-\alpha}$ for $|x| \leq \delta, \mu^{(\delta, 3)}(x) \leq \frac{\mathcal{A}_{\alpha}}{2 \pi(2+\alpha)} \delta^{-3-\alpha}$, for $\delta \leq|x| \leq 2 \delta$, and $\mu^{(\delta, 3)}(x)=0$ for $|x|>2 \delta$.

By Eqs. 19, 20 and 21, with $n=3$, we obtain

$$
g_{t}^{(\delta, 3)}(x) \leq c \frac{t}{\left(|x|+t^{1 / \alpha}\right)^{3+\alpha}}, \quad x \in \mathbb{R}^{3}, t \in(0, \tau]
$$


and

$$
g_{t}^{(\delta, 3)}(x) \leq c t^{1+\frac{d-1}{\alpha}} e^{-(\alpha+d)|x| / \alpha} \leq c \frac{t^{1+\frac{d-1}{\alpha}}}{\left(|x|+t^{1 / \alpha}\right)^{2}} e^{-|x|}, \quad|x|>\max \left\{\varepsilon, c_{3} t\right\}, t \in(0, \tau] .
$$

The above two inequalities yield

$$
g_{t}^{(\delta, 3)}(x) \leq c \frac{h_{t}^{(\varepsilon)}(|x|)}{\left(|x|+t^{1 / \alpha}\right)^{2}}, \quad x \in \mathbb{R}^{3}, t \in(0, \tau],
$$

and

$$
\left|\frac{d}{d r} g_{t}^{(\delta, 1)}(r)\right| \leq c \frac{r h_{t}^{(\varepsilon)}(r)}{\left(r+t^{1 / \alpha}\right)^{2}} \leq c \frac{h_{t}^{(\varepsilon)}(r)}{r+t^{1 / \alpha}}, \quad r>0, t \in(0, \tau] .
$$

Since $h_{t}^{(\varepsilon)}$ is nonincreasing, by the Lagrange theorem, we get

$$
\left|g_{t}^{(\delta, 1)}(x)-g_{t}^{(\delta, 1)}(y)\right| \leq c|x-y|\left(\frac{h_{t}^{(\varepsilon)}(|x|)}{|x|+t^{1 / \alpha}}+\frac{h_{t}^{(\varepsilon)}(|y|)}{|y|+t^{1 / \alpha}}\right), \quad x, y \in \mathbb{R} .
$$

Lemma 2.2 Let $\varepsilon \in(0,1]$. For any $t \in(0, \tau], x, x^{\prime} \in \mathbb{R}$, if $\left|x-x^{\prime}\right| \leq t^{1 / \alpha}$ and $\left|x-x^{\prime}\right| \leq$ $\varepsilon / 4$, then

$$
h_{t}^{(\varepsilon)}\left(x^{\prime}\right) \leq c h_{t}^{(\varepsilon)}(x / 2)
$$

Proof Assume first that $|x| \wedge\left|x^{\prime}\right| \leq \varepsilon / 2$. Then by the definition of $h_{t}^{(\varepsilon)}(x)$ we have $h_{t}^{(\varepsilon)}(x)=\frac{t}{\left(t^{1 / \alpha}+|x|\right)^{1+\alpha}} \geq \frac{t}{\left(t^{1 / \alpha}+\left|x-x^{\prime}\right|+\left|x^{\prime}\right|\right)^{1+\alpha}} \geq \frac{t}{\left(2 t^{1 / \alpha}+2\left|x^{\prime}\right|\right)^{1+\alpha}} \geq \operatorname{ch}_{t}^{(\varepsilon)}\left(x^{\prime}\right)$. Assume now that $|x| \wedge\left|x^{\prime}\right|>\varepsilon / 2$. Then we have $\left|x^{\prime}\right| \geq|x|-\left|x-x^{\prime}\right| \geq|x|-|x| / 2=|x| / 2$. Hence, $h_{t}^{(\varepsilon)}\left(x^{\prime}\right) \leq h_{t}^{(\varepsilon)}(x / 2)$.

Lemma 2.3 Let $\varepsilon \in(0,1], \delta=\varepsilon \min \left\{\frac{\alpha}{8(\alpha+d+2)}, \frac{1}{4 d\left(\eta_{1} \vee 1\right)^{2}}\right\}$. For any $t \in(0, \tau], x, x^{\prime} \in \mathbb{R}^{d}$, if $\left|x-x^{\prime}\right| \leq t^{1 / \alpha}$ and $\left|x-x^{\prime}\right| \leq \delta$, then

$$
\left|\prod_{i=1}^{d} g_{t}^{(\delta)}\left(x_{i}\right)-\prod_{i=1}^{d} g_{t}^{(\delta)}\left(x_{i}^{\prime}\right)\right| \leq c\left(\prod_{i=1}^{d} h_{t}^{(\varepsilon)}\left(x_{i} / 2\right)\right)\left[1 \wedge \sum_{j=1}^{d} t^{-1 / \alpha}\left|x_{j}-x_{j}^{\prime}\right|\right] .
$$

Proof By Lemma 2.1 we get

$$
\begin{aligned}
& \left|\prod_{i=1}^{d} g_{t}^{(\delta)}\left(x_{i}\right)-\prod_{i=1}^{d} g_{t}^{(\delta)}\left(x_{i}^{\prime}\right)\right| \\
& \leq \sum_{j=1}^{d}\left[\left|g_{t}^{(\delta)}\left(x_{j}\right)-g_{t}^{(\delta)}\left(x_{j}^{\prime}\right)\right| \prod_{i \neq j, 1 \leq i \leq d} g_{t}^{(\delta)}\left(\left|x_{i}\right| \wedge\left|x_{i}^{\prime}\right|\right)\right] \\
& \leq c\left(\prod_{i=1}^{d} h_{t}^{(\varepsilon)}\left(\left|x_{i}\right| \wedge\left|x_{i}^{\prime}\right|\right)\right) \sum_{j=1}^{d} \frac{\left|x_{j}-x_{j}^{\prime}\right|}{t^{1 / \alpha}} .
\end{aligned}
$$


Clearly, since $g_{t}^{(\delta)}$ is nonincreasing on $[0, \infty)$, we have

$$
\left|\prod_{i=1}^{d} g_{t}^{(\delta)}\left(x_{i}\right)-\prod_{i=1}^{d} g_{t}^{(\delta)}\left(x_{i}^{\prime}\right)\right| \leq \prod_{i=1}^{d} g_{t}^{(\delta)}\left(\left|x_{i}\right| \wedge\left|x_{i}^{\prime}\right|\right) .
$$

Now the assertion follows from Lemmas 2.1 and 2.2.

Lemma 2.4 Let $\varepsilon \in(0,1], \delta=\varepsilon \min \left\{\frac{\alpha}{8(\alpha+d+2)}, \frac{1}{4 d\left(\eta_{1} \vee 1\right)^{2}}\right\}$ and let $a \in \mathbb{R}$ be such that $|a| \leq \frac{\varepsilon}{4 \delta}$. Then there exists $c$ such that for any $t \in(0, \tau], x \in \mathbb{R}$ we have

$$
\int_{\mathbb{R}}\left|g_{t}^{(\delta)}(x+a w)-g_{t}^{(\delta)}(x)\right| \mu^{(\delta)}(w) d w \leq \frac{c|a|^{\alpha} h_{t}^{(\varepsilon)}\left(\frac{x}{2}\right)}{t} .
$$

Proof First we note that

$\int_{\mathbb{R}}\left|g_{t}^{(\delta)}(x+a w)-g_{t}^{(\delta)}(x)\right| \mu^{(\delta)}(w) d w \leq \int_{|w|<2 \delta}\left|g_{t}^{(\delta)}(x+a w)-g_{t}^{(\delta)}(x)\right| \mathcal{A}_{\alpha}|w|^{-1-\alpha} d w$, and by the substitution $s=a w$ we have

$$
\int_{|w|<2 \delta}\left|g_{t}^{(\delta)}(x+a w)-g_{t}^{(\delta)}(x)\right||w|^{-1-\alpha} d w=|a|^{\alpha} \int_{|s|<2 \delta|a|}\left|g_{t}^{(\delta)}(x+s)-g_{t}^{(\delta)}(x)\right||s|^{-1-\alpha} d s .
$$

Now we estimate the latter integral. Let

$$
\begin{aligned}
\int_{|s|<2 \delta|a|}\left|g_{t}^{(\delta)}(x+s)-g_{t}^{(\delta)}(x)\right||s|^{-1-\alpha} d s & =\int_{|s|<t^{1 / \alpha} \wedge(2 \delta|a|)}\left|g_{t}^{(\delta)}(x+s)-g_{t}^{(\delta)}(x)\right||s|^{-1-\alpha} d s \\
& +\int_{t^{1 / \alpha} \wedge(2 \delta|a|) \leq|s|<2 \delta|a|}\left|g_{t}^{(\delta)}(x+s)-g_{t}^{(\delta)}(x)\right||s|^{-1-\alpha} d s \\
& =: I_{1}+I_{2} .
\end{aligned}
$$

Using (18) we get

$$
\begin{aligned}
I_{1} & \leq c \int_{|s|<t^{1 / \alpha} \wedge(2 \delta|a|)}|s|^{-\alpha}\left(\frac{h_{t}^{(\varepsilon)}(x+s)}{t^{1 / \alpha}+|x+s|}+\frac{h_{t}^{(\varepsilon)}(x)}{t^{1 / \alpha}+|x|}\right) d s \\
& \leq c t^{-1 / \alpha} \int_{|s|<t^{1 / \alpha} \wedge(2 \delta|a|)}|s|^{-\alpha}\left(h_{t}^{(\varepsilon)}(|x+s|)+h_{t}^{(\varepsilon)}(|x|)\right) d s .
\end{aligned}
$$

If $|x| \geq 2 t^{1 / \alpha}$ then for $|s|<t^{1 / \alpha}$ we have $h_{t}^{(\varepsilon)}(|x+s|) \leq h_{t}^{(\varepsilon)}\left(\frac{|x|}{2}\right)$, since $h_{t}^{(\varepsilon)}$ is nonincreasing. If $|x| \leq 2 t^{1 / \alpha}$ and $|s|<t^{1 / \alpha}$ then $h_{t}^{(\varepsilon)}\left(\frac{|x|}{2}\right) \geq c t^{1 / \alpha}$ and $h_{t}^{(\varepsilon)}(|x+s|) \leq t^{1 / \alpha}$, hence we obtain $h_{t}^{(\varepsilon)}(|x+s|) \leq c h_{t}^{(\varepsilon)}\left(\frac{|x|}{2}\right)$. This yields

$$
I_{1} \leq c t^{-1 / \alpha} h_{t}^{(\varepsilon)}\left(\frac{|x|}{2}\right) \int_{|s|<t^{1 / \alpha}}|s|^{-\alpha} d s=\frac{c}{t} h_{t}^{(\varepsilon)}\left(\frac{|x|}{2}\right) .
$$

Now we estimate $I_{2}$. If $t^{1 / \alpha}>2 \delta|a|$ then $I_{2}=0$ so we assume that $t^{1 / \alpha} \leq 2 \delta|a|$ and using Eq. 17 we obtain

$$
I_{2} \leq c \int_{t^{1 / \alpha} \leq|s| \leq 2 \delta|a|}\left(h_{t}^{(\varepsilon)}(|x+s|)+h_{t}^{(\varepsilon)}(|x|)\right)|s|^{-1-\alpha} d s .
$$

Since we have

$$
\int_{|s| \geq t^{1 / \alpha}} h_{t}^{(\varepsilon)}(|x|)|s|^{-1-\alpha} d s=\frac{c}{t} h_{t}^{(\varepsilon)}(|x|)
$$


we only need to estimate

$$
J=\int_{t^{1 / \alpha} \leq|s| \leq 2 \delta|a|} h_{t}^{(\varepsilon)}(|x+s|)|s|^{-1-\alpha} d s .
$$

Let $g_{t}^{(\infty)}$ denote the transition density of the one-dimensional symmetric standard $\alpha$ stable process. It follows from [2] that $g_{t}^{(\infty)}(x) \approx \frac{t}{\left(t^{1 / \alpha}+|x|\right)^{1+\alpha}}$, hence $h_{t}^{(\varepsilon)}(y) \leq c g_{t}^{(\infty)}(y)$, for all $t \in(0, \tau], y \in \mathbb{R}$. Noting also that $|s|^{-1-\alpha} \leq c \frac{g_{t}^{(\infty)}(s)}{t}$ for $|s| \geq t^{1 / \alpha}$, and using the Chapman-Kolmogorov equation for $g_{t}^{(\infty)}$ we get

$$
\begin{aligned}
J & \leq \int_{|s| \geq t^{1 / \alpha}} h_{t}^{(\varepsilon)}(x+s)|s|^{-1-\alpha} d s \\
& \leq \frac{c}{t} \int_{\mathbb{R}} g_{t}^{(\infty)}(x+s) g_{t}^{(\infty)}(s) d s=\frac{c}{t} g_{2 t}^{(\infty)}(x),
\end{aligned}
$$

which yields $J \leq \frac{c h_{t}^{(\varepsilon)}(x)}{t}$ for $|x| \leq|\varepsilon|$.

Let now $|x| \geq \varepsilon \geq 4 \delta|a| \geq 2 t^{1 / \alpha}$. Then $|x+s| \geq|x| / 2$ for $s \leq 2 \delta|a|$, and we obtain

$$
\begin{aligned}
J & \leq \int_{t^{1 / \alpha} \leq|s| \leq 2 \delta|a|} h_{t}^{(\varepsilon)}(|x+s|)|s|^{-1-\alpha} d s \leq h_{t}^{(\varepsilon)}\left(\frac{|x|}{2}\right) \int_{t^{1 / \alpha} \leq|s|}|s|^{-1-\alpha} d s \\
& \leq \frac{c}{t} h_{t}^{(\varepsilon)}\left(\frac{|x|}{2}\right) .
\end{aligned}
$$

Lemma 2.5 For any $\varepsilon \in(0,1], \delta=\varepsilon \min \left\{\frac{\alpha}{8(\alpha+d+2)}, \frac{1}{4 d\left(\eta_{1} \vee 1\right)^{2}}\right\}$ and $m, n \in \mathbb{N}, n \geq 2$ there exists $c=c\left(m, n, \tau, \alpha, d, \eta_{1}, \eta_{2}, \eta_{3}, \varepsilon, \delta\right)$ such that for any $t \in(0, \tau], x \in \mathbb{R}$ we have

$$
\left|\frac{d^{m}}{d x^{m}} g_{t}^{(\delta)}(x)\right| \leq c t^{-(1+m) / \alpha}(1+|x|)^{-n} \text {. }
$$

Proof In the proof we assume that constants $c$ may additionally depend on $m$ and $n$. We use Theorem 3 of [16]. Let $f(s)=\mathcal{A}_{\alpha} s^{-1-\alpha}$ for $s \leq \delta$ and $f(s)=\mathcal{A}_{\alpha} \delta^{n-1-\alpha} s^{-n}$ for $s>\delta$. It is then obvious that the assumptions (1) and (2) of Theorem 3 in [16] hold and it follows that $\left|\frac{d^{m}}{d x^{m}} g_{t}^{(\delta)}(x)\right| \leq c t^{-(1+m) / \alpha} \min \left\{1, t^{1+1 / \alpha} f(|x|)+\left(1+\frac{|x|}{t^{1 / \alpha}}\right)^{-n}\right\}, \quad x \in \mathbb{R}, t \in(0, \tau]$.

Clearly, for $|x| \geq 1$ and $t \in(0, \tau]$ we have $f(|x|) \approx|x|^{-n}$ and $\left(1+\frac{|x|}{t^{1 / \alpha}}\right)^{-n} \leq c|x|^{-n}$. This implies the assertion of the lemma.

Lemma 2.6 There is a constant $C=C(\alpha)$ such that for $\delta \in(0,1], a \geq 0$, and any $t>0$,

$$
\int_{\mathbb{R}}\left(\frac{(a+|w|)|w|}{t^{1 / \alpha}} \wedge 1\right) \mu^{(\delta)}(w) d w \leq C \frac{t^{1 / 2}+a^{\alpha}}{t} .
$$

Proof We have

$$
\left(\frac{(a+|w|)|w|}{t^{1 / \alpha}} \wedge 1\right) \leq\left(\frac{a|w|}{t^{1 / \alpha}} \wedge 1\right)+\left(\frac{w^{2}}{t^{1 / \alpha}} \wedge 1\right)
$$


Hence, using $\mu^{(\delta)}(w) \leq \frac{\mathcal{A}_{\alpha}}{|w|^{1+\alpha}}$, we obtain

$\int_{\mathbb{R}}\left(\frac{(a+|w|)|w|}{t^{1 / \alpha}} \wedge 1\right) \mu^{(\delta)}(w) d w \leq \mathcal{A}_{\alpha} \int_{\mathbb{R}}\left(\frac{a|w|}{t^{1 / \alpha}} \wedge 1\right) \frac{d w}{|w|^{1+\alpha}}+\mathcal{A}_{\alpha} \int_{\mathbb{R}}\left(\frac{w^{2}}{t^{1 / \alpha}} \wedge 1\right) \frac{d w}{|w|^{1+\alpha}}$

Next,

and

$$
\int_{\mathbb{R}}\left(\frac{a|w|}{t^{1 / \alpha}} \wedge 1\right) \frac{d w}{|w|^{1+\alpha}}=\frac{a^{\alpha}}{t} \int_{\mathbb{R}}(|w| \wedge 1) \frac{d w}{|w|^{1+\alpha}}
$$

$$
\int_{\mathbb{R}}\left(\frac{w^{2}}{t^{1 / \alpha}} \wedge 1\right) \frac{d w}{|w|^{1+\alpha}}=t^{-1 / 2} \int_{\mathbb{R}}\left(w^{2} \wedge 1\right) \frac{d w}{|w|^{1+\alpha}}
$$

In the sequel we will need a version of the inverse map theorem for a Lipschitz function $f: \mathbb{R}^{n} \rightarrow \mathbb{R}^{n}, n \in \mathbb{N}$. The corresponding theorem is the main result in [9], however it is not formulated in a suitable way for our purpose. Below, closely following the arguments from [9], we provide a version we need.

It is well known that $y$ almost surely the Jacobi matrix $\mathcal{J}_{f}(y)$ of $f$ exists. For any $y_{0} \in$ $\mathbb{R}^{n}$ we define (see Definition 1 in [9]) the generalized Jacobian denoted $\partial f\left(y_{0}\right)$ as the convex hull of the set of matrices which can be obtained as limits of $\mathcal{J}_{f}\left(y_{n}\right)$, when $y_{n} \rightarrow y_{0}$.

We denote by $B(x, r)$ an open ball of the center $x \in \mathbb{R}^{n}$ and radius $r>0$. For any matrix $M$ we denote by $\|M\|_{\infty}$ the maximum of its entries.

Lemma 2.7 Let $f: \mathbb{R}^{n} \rightarrow \mathbb{R}^{n}$ be a Lipschitz map and $x \in \mathbb{R}^{n}$. Suppose that for any $y \in \mathbb{R}^{n}$, the generalized Jacobian $\partial f(y)$ consist of the matrices which can be represented as $M(x)+R$, where matrices $M(x), R$ satisfy the following conditions: there are positive $\beta$ and $\eta$ such that $\|R\|_{\infty} \leq \eta|x-y|$ and $\left|v M(x)^{T}\right| \geq 2 \beta$ for every $v \in \mathbb{R}^{n},|v|=1$. Then $f$ is injective on $B(x, \beta /(n \eta))$ and we have $B\left(f(x), \beta^{2} /(2 n \eta)\right) \subset f(B(x, \beta /(n \eta)))$.

Proof Let $v$ be an arbitrary unit vector in $\mathbb{R}^{n}$. Let $M \in \partial f(y)$ and let $z=v M(x)^{T}$. Since $M^{T}=M(x)^{T}+R^{T}$ the scalar product of $z$ and $w=v M^{T}=z+v R^{T}$ can be estimated as follows

$$
z \cdot w=z \cdot\left(z+v R^{T}\right)=|z|^{2}+z \cdot\left(v R^{T}\right) \geq|z|^{2}-n \eta|z||x-y| .
$$

Next, taking $w^{*}=z /|z|$ we have for $|x-y| \leq \beta /(n \eta)$,

$$
w^{*} \cdot\left(v M^{T}\right) \geq|z|-n \eta|x-y| \geq \beta .
$$

Using this fact we can apply Lemma 3 and Lemma 4 of [9] to claim that for every $y_{1}, y_{2} \in$ $B(x, \beta /(n \eta))$ we have

$$
\left|f\left(y_{1}\right)-f\left(y_{2}\right)\right| \geq \beta\left|y_{1}-y_{2}\right|,
$$

which shows that $f$ is injective in a ball $B(x, \beta /(n \eta))$. Next, by similar arguments, we show that

$$
\left|v M^{T}\right| \geq\left|v M(x)^{T}\right|-\left|v R^{T}\right| \geq 2 \beta-n \eta|x-y| \geq \beta,|y-x| \leq \beta /(n \eta),
$$

which proves that all matrices from the set $\partial f(y)$ are of full rank if $|y-x| \leq \beta /(n \eta)$.

Finally, we can apply Lemma 5 of [9] to show that the $f$ image of the ball $B(x, \beta /(n \eta))$ contains the ball $B\left(f(x), \beta^{2} /(2 n \eta)\right)$. 


\section{Construction and Properties of the Transition Density of the Solution of (1) Driven by the Truncated Process}

The approach in this section is based on Levi's method (cf. [12, 26, 29]). This method was applied in the framework of pseudodifferential operators by Kochubei [20] to construct a fundamental solution to the related Cauchy problem as well as transition density for the corresponding Markow process. In recent years it was used in several papers to study transition densities of Lévy-type processes see e.g. [5, 7, 8, 13, 15, 17-19, 21]. Levi's method was also used to study gradient and Schrödinger perturbations of fractional Laplacians see e.g. $[4,6,41]$.

Let us fix $\varepsilon \in(0,1]$ (it will be chosen later). Recall that for given $\varepsilon$ the constant $\delta$ is chosen according to Lemma 2.1. For such fixed $\varepsilon, \delta$ we abbreviate $\mu(x)=\mu^{(\delta)}(x)$, $\mathcal{G}=\mathcal{G}^{(\delta)}, g_{t}(x)=g_{t}^{(\delta)}(x), h_{t}(x)=h_{t}^{(\varepsilon)}(x), x \in \mathbb{R}$. Note that $h_{t}^{(\varepsilon)}$ depends also on $\tau>0$ which we keep fixed.

Let us recall that

$$
\mathcal{L} f(x)=\sum_{i=1}^{d} \lim _{\zeta \rightarrow 0^{+}} \int_{|w|>\zeta}\left[f\left(x+a_{i}(x) w\right)-f(x)\right] \mu(w) d w .
$$

Our first aim in this section will be to construct the heat kernel $u(t, x, y)$ corresponding to the operator $\mathcal{L}$. This will be done by using Levi's method.

Let $G_{t}(x)=g_{t}\left(x_{1}\right) \ldots g_{t}\left(x_{d}\right)$ and $H_{t}(x)=h_{t}\left(x_{1}\right) \ldots h_{t}\left(x_{d}\right)$ for $t>0$ and $x=$ $\left(x_{1}, \ldots, x_{d}\right) \in \mathbb{R}^{d}$. We also denote $B(x)=\left(b_{i j}(x)\right)=A^{-1}(x)$. Note that the coordinates of $B(x)$ satisfy the conditions (2) and (4) with possibly different constants $\eta_{1}^{*}$ and $\eta_{3}^{*}$, but taking maximums we can assume that $\eta_{1}^{*}=\eta_{1}$ and $\eta_{3}^{*}=\eta_{3}$.

For any $y \in \mathbb{R}^{d}, i=1, \ldots, d$ we put

$$
b_{i}(y)=\left(b_{i 1}(y), \ldots, b_{i d}(y)\right) .
$$

We also denote $\|B\|_{\infty}=\max \left\{\left|b_{i j}\right|: i, j \in\{1, \ldots, d\}\right\}$. For any $t>0, x, y \in \mathbb{R}^{d}$ we define

$$
\begin{aligned}
p_{y}(t, x) & =\operatorname{det}(B(y)) G_{t}\left(x(B(y))^{T}\right) \\
& =\operatorname{det}(B(y)) g_{t}\left(b_{1}(y) \cdot x\right) \ldots g_{t}\left(b_{d}(y) \cdot x\right) .
\end{aligned}
$$

Recall that $\mathcal{L}^{y}$ is the "freezing" operator given by (11). It may be easily checked that for each fixed $y \in \mathbb{R}^{d}$ the function $p_{y}(t, \cdot)$ is the heat kernel of $\mathcal{L}^{y}$ that is

$$
\begin{gathered}
\frac{\partial}{\partial t} p_{y}(t, x)=\mathcal{L}^{y} p_{y}(t, \cdot)(x), \quad t>0, x \in \mathbb{R}^{d}, \\
\int_{\mathbb{R}^{d}} p_{y}(t, x) d x=1, \quad t>0 .
\end{gathered}
$$

For any $t>0, x, y \in \mathbb{R}^{d}$ we also define

$$
\begin{aligned}
r_{y}(t, x) & =H_{t}\left(x(B(y))^{T}\right) \\
& =h_{t}\left(b_{1}(y) \cdot x\right) \ldots h_{t}\left(b_{1}(y) \cdot x\right) .
\end{aligned}
$$

Now we use the approach which was explained in details in the Introduction. The approximation $\tilde{u}(t, x, y)$ of $u(t, x, y)$ is defined by $\tilde{u}(t, x, y)=p_{y}(t, x-y)$. For $x, y \in \mathbb{R}^{d}, t>0$, the kernel $q_{0}(t, x, y)$ is given by (8). We have

$$
\mathcal{L} \tilde{u}(t, \cdot, y)(x)=\mathcal{L}^{x} p_{y}(t, \cdot)(x-y),
$$


hence by (24) we get

$$
q_{0}(t, x, y)=\mathcal{L}^{x} p_{y}(t, \cdot)(x-y)-\mathcal{L}^{y} p_{y}(t, \cdot)(x-y) .
$$

For $x, y \in \mathbb{R}^{d}, t>0$ and $n \in \mathbb{N}$ kernels $q_{n}(t, x, y)$ are given by (10). For $x, y \in \mathbb{R}^{d}, t>0$ we define

$$
q(t, x, y)=\sum_{n=0}^{\infty} q_{n}(t, x, y) .
$$

The kernel $u(t, x, y)$ is given by (9). We have

$$
u(t, x, y)=p_{y}(t, x-y)+\int_{0}^{t} \int_{\mathbb{R}^{d}} p_{z}(t-s, x-z) q(s, z, y) d z d s .
$$

In this section we will show that $q_{n}(t, x, y), q(t, x, y), u(t, x, y)$ are well defined and we will obtain estimates of these functions. First, we will get some simple properties of $p_{y}(t, x)$ and $r_{y}(t, x)$.

Lemma 3.1 Choose $\gamma \in(0,1]$. For any $t \in(0, \tau], x, x^{\prime}, y \in \mathbb{R}^{d}$ we have

$$
\left|p_{y}(t, x)-p_{y}\left(t, x^{\prime}\right)\right| \leq c\left(1 \wedge\left(t^{-\gamma / \alpha}\left|x-x^{\prime}\right|^{\gamma}\right)\right)\left(r_{y}(t, x / 2)+r_{y}\left(t, x^{\prime} / 2\right)\right) .
$$

Proof Of course, we may assume that $\gamma=1$. We have

$$
p_{y}(t, x)-p_{y}\left(t, x^{\prime}\right)=\operatorname{det}(B(y))\left(\prod_{i=1}^{d} g_{t}\left(b_{i}(y) \cdot x\right)-\prod_{i=1}^{d} g_{t}\left(b_{i}(y) \cdot x^{\prime}\right)\right)
$$

If $\left|x-x^{\prime}\right| \geq t^{1 / \alpha} /\|B\|_{\infty}$ or $\left|x-x^{\prime}\right| \geq \delta /\|B\|_{\infty}$ then the assertion clearly holds. So, we may assume that $\left|x-x^{\prime}\right| \leq t^{1 / \alpha} /\|B\|_{\infty}$ and $\left|x-x^{\prime}\right| \leq \delta /\|B\|_{\infty}$. Then the assertion follows easily from Lemma 2.3.

For $x, y \in \mathbb{R}^{d}$ we have

$$
\left|B(y) x^{T}\right|^{2}=\left|x B(y)^{T}\right|^{2}=\left(b_{1}(y) \cdot x\right)^{2}+\ldots+\left(b_{1}(y) \cdot x\right)^{2} .
$$

Lemma 3.2 For any $x, y \in \mathbb{R}^{d}$ and $i \in\{1, \ldots, d\}$ we have

$$
\max _{1 \leq i \leq d}\left(b_{i}(y) \cdot x\right)^{2} \geq \frac{1}{\eta_{1}^{2} d^{3}}|x|^{2} .
$$

Proof Indeed, for any $u, x \in \mathbb{R}^{d}$ we have $\left|u A(y)^{T}\right| \leq \eta_{1} d|u|$. Setting $u=x B(y)^{T}$ we obtain that

$$
\left|x B(y)^{T}\right| \geq \frac{1}{\eta_{1} d}|x| .
$$

Since

$$
\left|x B(y)^{T}\right|^{2}=\left|b_{1}(y) \cdot x\right|^{2}+\left|b_{2}(y) \cdot x\right|^{2}+\cdots+\left|b_{1}(y) \cdot x\right|^{2},
$$

it follows that there is $1 \leq k \leq d$ such that $\left|b_{k}(y) \cdot x\right| \geq \frac{1}{\eta_{1} d \sqrt{d}}|x|$.

Corollary 3.3 Assume that $\varepsilon \leq \frac{1}{\eta_{1} d \sqrt{d}}$. For any $t \in(0, \tau+1], x, y \in \mathbb{R}^{d}$, we have

$$
r_{y}(t, x-y) \leq c_{1} t^{-d / \alpha} e^{-c|x-y|} .
$$


For any $t \in(0, \tau+1], x, y \in \mathbb{R}^{d},|x-y| \geq \varepsilon \eta_{1} d^{3 / 2}$, we have

$$
r_{y}(t, x-y) \leq c_{1} t e^{-c|x-y|} \text {. }
$$

Proof For any $t \in(0, \tau+1], z \in \mathbb{R}$, by definition of $h_{t}$, we have

$$
h_{t}(z) \leq c t^{-1 / \alpha} e^{-|z|} \text {. }
$$

Fix $x, y \in \mathbb{R}^{d}, t \in(0, \tau+1]$. By Lemma 3.2, there exists $i \in\{1, \ldots, d\}$ such that $\left|b_{i}(y) \cdot(x-y)\right| \geq \frac{1}{\eta_{1} d \sqrt{d}}|x-y|$. Using this and (28) we get (26). For any $t \in(0, \tau+1]$, $z \in \mathbb{R},|z| \geq \varepsilon$, by definition of $h_{t}$, we have

$$
h_{t}(z) \leq c t^{1+(d-1) / \alpha} e^{-|z|} .
$$

If $|x-y| \geq \varepsilon \eta_{1} d^{3 / 2}$ then $\frac{1}{\eta_{1} d \sqrt{d}}|x-y| \geq \varepsilon$ hence, by the same arguments as above, we get (27).

Using the definition of $p_{y}(t, x)$ and properties of $g_{t}(x)$ we obtain the following regularity properties of $p_{y}(t, x)$.

Lemma 3.4 The function $(t, x, y) \rightarrow p_{y}(t, x)$ is continuous on $(0, \infty) \times \mathbb{R}^{d} \times \mathbb{R}^{d}$. The function $t \rightarrow p_{y}(t, x)$ is in $C^{1}((0, \infty))$ for each fixed $x, y \in \mathbb{R}^{d}$. The function $x \rightarrow p_{y}(t, x)$ is in $C^{2}\left(\mathbb{R}^{d}\right)$ for each fixed $t>0, y \in \mathbb{R}^{d}$.

Lemma 3.5 For any $y \in \mathbb{R}^{d}$ we have

$$
\begin{gathered}
\left|\frac{\partial}{\partial x_{i}} p_{y}(t, x-y)\right| \leq \frac{c}{t^{(d+1) / \alpha}(1+|x-y|)^{d+1}}, \quad i \in\{1, \ldots, d\}, t \in(0, \tau], x \in \mathbb{R}^{d}, \\
\left|\frac{\partial^{2}}{\partial x_{i} \partial x_{j}} p_{y}(t, x-y)\right| \leq \frac{c}{t^{(d+2) / \alpha}(1+|x-y|)^{d+1}}, \quad i, j \in\{1, \ldots, d\}, t \in(0, \tau], x \in \mathbb{R}^{d} .
\end{gathered}
$$

Proof The estimates follow from Lemma 2.5 and the same arguments as in the proof of (26).

Lemma 3.6 Let $b_{i}^{*}(x, y), x, y \in \mathbb{R}^{d} ; i=1, \ldots, d$, be real functions such that there are positive $\eta_{4}, \eta_{5}$ and

$$
\begin{gathered}
\left|b_{i}^{*}(x, y)\right| \leq \eta_{4}, \quad x, y \in \mathbb{R}^{d}, \\
\left|b_{i}^{*}(x, y)-b_{i}^{*}(\bar{x}, \bar{y})\right| \leq \eta_{5}(|x-\bar{x}|+|y-\bar{y}|), \quad x, y, \bar{x}, \bar{y} \in \mathbb{R}^{d} .
\end{gathered}
$$

Let, for $i=1, \ldots, d$,

$$
\xi_{i}=\xi_{i}(w, x, y)=b_{i}(y) \cdot(x-y)+b_{i}^{*}(x, y) w, \quad w \in \mathbb{R}, x, y \in \mathbb{R}^{d} .
$$

We put

$$
\Psi(w, x, y)=\left(w, \xi_{1}, \ldots, \xi_{d}\right) \in \mathbb{R}^{d+1}, \quad w \in \mathbb{R}, x, y \in \mathbb{R}^{d}
$$

and for fixed $x \in \mathbb{R}^{d}$ we define a map $\Psi_{x}: \mathbb{R}^{d+1} \mapsto \mathbb{R}^{d+1}$ by $\Psi_{x}=\Psi(\cdot, x, \cdot)$, while for fixed $y \in \mathbb{R}^{d}$ we define a map $\Phi_{y}: \mathbb{R}^{d+1} \mapsto \mathbb{R}^{d+1}$ by $\Phi_{y}=\Psi(\cdot, \cdot, y)$.

There is a positive $\varepsilon_{0}=\varepsilon_{0}\left(\eta_{1}, \eta_{3}, \eta_{4}, \eta_{5}, d\right) \leq \frac{1}{2 \eta_{5}}$ such that the map $\Psi_{x}$ and its Jacobian determinant denoted by $J_{\Psi_{x}}$ has the property

$$
\begin{aligned}
\left|\Psi_{x}(w, y)\right| & \leq 1, \\
(1 / 2)|\operatorname{det} B(y)| \leq\left|J_{\Psi_{x}}(w, y)\right| & \leq 2|\operatorname{det} B(y)|,
\end{aligned}
$$


$(w, y)$ almost surely on the set $Q_{x}=\left\{(w, y) \in \mathbb{R}^{d+1}:|y-x| \leq \varepsilon_{0},|w| \leq \varepsilon_{0}\right\}$. The map $\Psi_{x}$ is injective on $Q_{x}$.

Morever, the map $\Phi_{y}$ and its Jacobian determinant denoted by $J_{\Phi_{y}}$ has the property

$$
\begin{aligned}
\left|\Phi_{y}(w, x)\right| & \leq 1, \\
(1 / 2)|\operatorname{det} B(x)| \leq\left|J_{\Phi_{y}}(w, x)\right| & \leq 2|\operatorname{det} B(x)|,
\end{aligned}
$$

$(w, x)$ almost surely on the set $Q_{y}=\left\{(w, x) \in \mathbb{R}^{d+1}:|y-x| \leq \varepsilon_{0},|w| \leq \varepsilon_{0}\right\}$. The map $\Phi_{y}$ is injective on $Q_{y}$.

Proof In the proof we assume that constants $c$ may additionally depend on $\eta_{4}, \eta_{5}$. We prove the statement for the map $\Psi_{x}$, only. Since $\left|\Psi_{x}(w, y)\right| \leq \sqrt{d}\left(1+\eta_{1}+\eta_{4}\right)(|w|+|x-y|)$ we have

$$
\left|\Psi_{x}(w, y)\right| \leq 1, \text { if }|w|+|x-y| \leq \frac{1}{\sqrt{d}\left(1+\eta_{1}+\eta_{4}\right)} .
$$

Next, we observe that $(w, y)$ almost surely

$$
\frac{\partial \xi_{k}}{\partial y_{l}}=-b_{k l}(y)+(x-y) \cdot \frac{\partial b_{k}}{\partial y_{l}}+w \frac{\partial b_{k}^{*}}{\partial y_{l}}, \quad 1 \leq l, k \leq d .
$$

Since $\left|(y-x) \cdot \frac{\partial b_{k}}{\partial y_{l}}+w \frac{\partial b_{k}^{*}}{\partial y_{l}}\right| \leq\left(\eta_{3}+\eta_{5}\right)(|y-x|+|w|)$, it follows that

$$
J_{\Psi_{x}}(w, y)=(-1)^{d} \operatorname{det} B(y)+R(x, y, w), \quad|R(x, y, w)| \leq c(|y-x|+|w|),
$$

with $c=c\left(d, \eta_{1}, \eta_{3}, \eta_{5}\right)$. Since $|\operatorname{det} B(y)|=\frac{1}{|\operatorname{det} A(y)|} \geq \frac{1}{d ! \eta_{1}^{d}}$, we have for sufficiently small $\varepsilon_{1}=\varepsilon_{1}\left(\eta_{1}, \eta_{3}, \eta_{4}, \eta_{5}, d\right),(w, y)$ almost surely

$$
J_{\Psi_{x}}(w, y)=(-1)^{d} \kappa(x, y, w) \operatorname{det} B(y),|y-x| \leq \varepsilon_{1},|w| \leq \varepsilon_{1},
$$

where $\frac{1}{2} \leq \kappa \leq 2$.

Let $\mathcal{J}_{\Psi_{x}}(w, y)$ be the Jacobi matrix for the map $\Psi_{x}$ which is defined $(w, y)$ almost surely. From (32) it follows that

$$
\mathcal{J}_{\Psi_{x}}(w, y)=\mathcal{B}(x)+\mathcal{R},
$$

where the coordinates $\mathcal{B}_{k l}(x), 0 \leq k, l \leq d$ of the matrix $\mathcal{B}(x)$ are

$$
\begin{aligned}
& \mathcal{B}_{k l}(x)=-b_{k l}(x), \quad k, l \geq 1, \\
& \mathcal{B}_{00}(x)=1 ; \mathcal{B}_{0 l}(x)=0 ; \mathcal{B}_{l 0}(x)=b_{l}^{*}(x, x), \quad 1 \leq l \leq d,
\end{aligned}
$$

while all the entries of $\mathcal{R}$ satisfy $\left|\mathcal{R}_{k l}\right| \leq c \sqrt{|w|^{2}+|x-y|^{2}}$ with $c=c\left(\eta_{3}, \eta_{5}\right)$.

Let $\partial \Psi_{x}(w, y)$ denote the generalized Jacobian of $\Psi_{x}$ at the point $(w, y)$. Then from the form of $\mathcal{J}_{\Psi_{x}}$ it is clear that every matrix $\mathcal{M} \in \partial \Psi_{x}(w, y)$ can be written as

$$
\mathcal{M}=\mathcal{B}(x)+\mathcal{R}
$$

where all the entries of $\mathcal{R}$ satisfy $\left|\mathcal{R}_{k l}\right| \leq c \sqrt{|w|^{2}+|x-y|^{2}}$ with $c=c\left(\eta_{3}, \eta_{5}\right)$.

Now, for every $(u, z), u \in \mathbb{R}, z \in \mathbb{R}^{\bar{d}}:|u|^{2}+|z|^{2}=1$ we have

$$
\left|(u, z) \mathcal{B}(x)^{T}\right| \geq 2 \beta>0,
$$

with $\beta=\beta\left(d, \eta_{1}, \eta_{4}\right)$. Since $\|\mathcal{R}\|_{\infty} \leq c \sqrt{|w|^{2}+|x-y|^{2}}$ we can apply Lemma 2.7 with $n=d+1$ to show on the set $\left\{(w, y) ; \sqrt{|w|^{2}+|x-y|^{2}} \leq \beta /(c(d+1))\right\}$ the map $\Psi_{x}$ is injective. This fact, combined with (31) and (33), completes the proof if we choose $\varepsilon_{0}=$ $\varepsilon_{1} \wedge \frac{1}{2 \sqrt{d}\left(1+\eta_{1}+\eta_{4}\right)} \wedge \frac{\beta}{2(d+1) c}$. 
Remark 3.7 Let for $x \in \mathbb{R}^{d}, \tilde{\Psi}_{x}$ be the map $\mathbb{R}^{d} \mapsto \mathbb{R}^{d}$ given by

$$
\tilde{\Psi}_{x}(y)=\left(\xi_{1}, \ldots, \xi_{d}\right) \in \mathbb{R}^{d}, \quad y \in \mathbb{R}^{d},
$$

where $\xi_{i}=b_{i}(y) \cdot(x-y)$. Then using the same arguments as in the above proof we can find $\varepsilon_{0}$ such that all the assertions of Lemma 3.6 are true and additionally

$$
(1 / 2)|\operatorname{det} B(y)| \leq\left|J_{\tilde{\Psi}_{x}}(y)\right| \leq 2|\operatorname{det} B(y)|,
$$

for $|x-y| \leq \varepsilon_{0}, y$ almost surely. Moreover, the map $\tilde{\Psi}_{x}$ is injective on $B\left(x, \varepsilon_{0}\right)$. We can also find $\delta_{1}=\delta_{1}\left(\eta_{1}, \eta_{3}, \eta_{4}, \eta_{5}, d\right)>0$ and $\delta_{2}=\delta_{1}\left(\eta_{1}, \eta_{3}, \eta_{4}, \eta_{5}, d\right)>0$ such that the $\tilde{\Psi}_{x}$ image of the ball $B\left(x, \delta_{1}\right)$ contains $B\left(0, \delta_{2}\right)$. To this end we apply the last assertion of Lemma 2.7.

Let $b_{i}^{*}(x, y)$ be the functions introduced in Lemma 3.6. We will use the following abbreviations

$$
\begin{aligned}
& z_{i}=B_{i}(x, y)=b_{i}(y) \cdot(x-y)=b_{i 1}(y)\left(x_{1}-y_{1}\right)+\ldots+b_{i d}(y)\left(x_{d}-y_{d}\right), \\
& b_{i}^{*}=b_{i}^{*}(x, y) \\
& b_{i 0}^{*}=b_{i}^{*}(x, x) .
\end{aligned}
$$

Let for $l=1, \ldots, d$,

$$
\mathrm{A}_{l}=\mathrm{A}_{l}(x, y)=\int_{\mathbb{R}} \prod_{i \neq l} g_{t}\left(z_{i}+b_{i}^{*} w\right)\left|g_{t}\left(z_{l}+b_{l}^{*} w\right)-g_{t}\left(z_{l}+b_{l 0}^{*} w\right)\right| \mu(w) d w .
$$

Corollary 3.8 Assume that $2 \delta<\varepsilon_{0}$, where $\varepsilon_{0}$ is from Remark 3.7. With the assumptions of Lemma 3.6 we have for $t \leq \tau$,

$$
\int_{|y-x| \leq \varepsilon_{0}} A_{l} d y \leq c t^{-1 / 2}, \quad x \in \mathbb{R}^{d}
$$

and

$$
\int_{|y-x| \leq \varepsilon_{0}} A_{l} d x \leq c t^{-1 / 2}, \quad y \in \mathbb{R}^{d}
$$

where $c=c\left(\tau, \alpha, d, \eta_{1}, \eta_{2}, \eta_{3}, \eta_{4}, \eta_{5}, \varepsilon\right)$.

Proof In the proof we assume that constants $c$ may additionally depend on $\eta_{4}, \eta_{5}$. For $x, y \in$ $\mathbb{R}^{d}$ we get $\left|b_{l}^{*}-b_{l 0}^{*}\right| \leq \eta_{5}|x-y|$. Hence, from (18), we have for $w \in \mathbb{R}, 1 \leq l \leq d$,

$$
\begin{aligned}
\left|g_{t}\left(z_{l}+b_{l}^{*} w\right)-g_{t}\left(z_{l}+b_{l 0}^{*} w\right)\right| & \leq c\left(\frac{\left|b_{l}^{*}-b_{l 0}^{*}\right||w|}{t^{1 / \alpha}} \wedge 1\right)\left(h_{t}\left(z_{l}+b_{l}^{*} w\right)+h_{t}\left(z_{l}+b_{l 0}^{*} w\right)\right) \\
\leq & c\left(\frac{|x-y||w|}{t^{1 / \alpha}} \wedge 1\right)\left(g_{t}^{(\infty)}\left(z_{l}+b_{l}^{*} w\right)+g_{t}^{(\infty)}\left(z_{l}+b_{l 0}^{*} w\right)\right) .
\end{aligned}
$$

This implies that

$$
\mathrm{A}_{l} \leq c\left(\mathrm{~A}_{l}^{1}+\mathrm{A}_{l}^{2}\right)
$$

where

$$
\mathrm{A}_{l}^{1}=\int_{\mathbb{R}}\left(\prod_{i=1}^{d} g_{t}^{(\infty)}\left(z_{i}+b_{i}^{*} w\right)\right)\left(\frac{|x-y||w|}{t^{1 / \alpha}} \wedge 1\right) \mu(w) d w
$$


and

$$
\mathrm{A}_{l}^{2}=\int_{\mathbb{R}}\left(\prod_{i=1}^{d} g_{t}^{(\infty)}\left(z_{i}+\hat{b}_{i} w\right)\right)\left(\frac{|x-y||w|}{t^{1 / \alpha}} \wedge 1\right) \mu(w) d w
$$

with $\hat{b}_{i}=b_{i}^{*}, i \neq l$ and $\hat{b}_{l}=b_{l 0}^{*}$. Note that the functions $\hat{b}_{i}=\hat{b}_{i}(x, y)$ have the same properties $(29,30)$ as $b_{i}^{*}$. To evaluate the integral $\int_{|x-y| \leq \varepsilon_{0}} \mathrm{~A}_{l}^{1} d y$, which in fact is an integral with respect to $d w d y$, we introduce new variables $(w, \xi)$ in $\mathbb{R}^{d+1}$, given by $(w, \xi)=$ $\Psi_{x}(w, y)$, where $\xi_{i}=z_{i}+b_{i}^{*} w, i=1, \ldots, d$ ( or $\xi_{i}=z_{i}+\hat{b}_{i} w$ if $\mathrm{A}_{l}^{2}$ is treated). We recall that $\Psi_{x}$ was defined in Lemma 3.6. Note that the vector $\xi=\left(\xi_{1}, \ldots, \xi_{d}\right)$ can be written as

$$
\xi=(x-y) B(y)^{T}+w b^{*},
$$

where $b^{*}=\left(b_{1}^{*}, \ldots, b_{d}^{*}\right)$, hence

$$
\left(\xi-w b^{*}\right) A(y)^{T}=x-y .
$$

From this we infer that

$$
|w||x-y| \leq c(|\xi|+|w|)|w| .
$$

Let $Q_{x}=\left\{(w, y):|y-x| \leq \varepsilon_{0},|w| \leq \varepsilon_{0}\right\}$. Due to Lemma 3.6, almost surely on $Q_{x}$, the absolute value of the Jacobian determinant of the map $\Psi_{x}$ is bounded from below and above by two positive constants and $\Psi_{x}$ is an injective transformation. Let $V_{x}=\Psi_{x}\left(Q_{x}\right)$. Observing that the support of the density $\mu$ is contained in $\left[-\varepsilon_{0}, \varepsilon_{0}\right]$ and then applying the above change of variables, we have

$$
\begin{aligned}
\int_{|y-x| \leq \varepsilon_{0}} \mathrm{~A}_{l}^{1} d y \leq & c \int_{|y-x| \leq \varepsilon_{0}} \int_{\mathbb{R}}\left(\prod_{i=1}^{d} g_{t}^{(\infty)}\left(\xi_{i}\right)\right)\left(\frac{(|\xi|+|w|)|w|}{t^{1 / \alpha}} \wedge 1\right) \mu(w) d w d y \\
\leq & c \int_{|y-x| \leq \varepsilon_{0}} \int_{\mathbb{R}}\left(\prod_{i=1}^{d} g_{t}^{(\infty)}\left(\xi_{i}\right)\right)\left(\frac{(|\xi|+|w|)|w|}{t^{1 / \alpha}} \wedge 1\right) \\
& \times \mu(w)\left|J_{\Psi_{x}}(w, y)\right| d w d y \\
= & c \int_{V_{x}} \prod_{i=1}^{d} g_{t}^{(\infty)}\left(\xi_{i}\right)\left(\frac{(|\xi|+|w|)|w|}{t^{1 / \alpha}} \wedge 1\right) \mu(w) d w d \xi
\end{aligned}
$$

where the last equality follows from the general change of variable formula for injective Lipschitz maps (see e.g. [14, Theorem 3]). Since $|\xi| \leq 1$ for $(w, \xi) \in V_{x}$, we get

$$
\int_{|y-x| \leq \varepsilon_{0}} \mathrm{~A}_{l}^{1} d y \leq c \int_{|\xi| \leq 1} \prod_{i=1}^{d} g_{t}^{(\infty)}\left(\xi_{i}\right) \int_{\mathbb{R}}\left(\frac{(|\xi|+|w|)|w|}{t^{1 / \alpha}} \wedge 1\right) \mu(w) d w d \xi .
$$

Applying Lemma 2.6 we have

$$
\int_{\mathbb{R}}\left(\frac{(|\xi|+|w|)|w|}{t^{1 / \alpha}} \wedge 1\right) \mu(w) d w \leq c \frac{t^{1 / 2}+|\xi|^{\alpha}}{t}
$$

Finally,

$$
\int_{|y-x| \leq \varepsilon_{0}} \mathrm{~A}_{l}^{1} d y \leq c \int_{|\xi| \leq 1} \prod_{i=1}^{d} g_{t}^{(\infty)}\left(\xi_{i}\right) \frac{t^{1 / 2}+|\xi|^{\alpha}}{t} d \xi \leq c t^{-1 / 2} .
$$

Similarly we obtain

$$
\int_{|y-x| \leq \varepsilon_{0}} \mathrm{~A}_{l}^{2} d y \leq c t^{-1 / 2}
$$


which completes the proof of the first bound. To estimate $\int_{|y-x| \leq \varepsilon_{0}} \mathrm{~A}_{l} d x$ we proceed exactly in the same way.

For fixed $l \in\{1, \ldots, d\}$ let us consider a family of functions $b_{i}^{*}(x, y)=b_{i}(y) \cdot a_{l}(x), i \in$ $\{1, \ldots, d\}$. They satisfy the conditions (29) and (30) with $\eta_{4}=d \eta_{1}^{2}$ and $\eta_{5}=d \eta_{1} \eta_{3}$. Let $\varepsilon_{0}=\varepsilon_{0}\left(\eta_{1}, \eta_{3}, \eta_{4}, \eta_{5}, d\right)$ be as found in Lemma 3.6 and Remark 3.7. Finally we choose $\varepsilon=\varepsilon\left(\eta_{1}, \eta_{3}, d\right)=\frac{\varepsilon_{0}}{4 d^{3 / 2}\left(\eta_{1} \vee 1\right)}$. From now on we keep $\varepsilon_{0}, \varepsilon$ fixed as above. Recall that if we fixed $\varepsilon$ we fix $\delta$ according to Lemma 2.1.

Proposition 3.9 For any $x, y \in \mathbb{R}^{d}, t \in(0, \tau]$ we have

$$
\left|q_{0}(t, x, y)\right| \leq c t^{-1-(d-1) / \alpha} h_{t}\left(\left(\varepsilon / \varepsilon_{0}\right)|x-y| \mathbf{1}_{\left[\varepsilon_{0}, \infty\right)}(|x-y|)\right) .
$$

In particular, for $x, y \in \mathbb{R}^{d}, t \in(0, \tau],|y-x| \geq \varepsilon_{0}$ we have

$$
\left|q_{0}(t, x, y)\right| \leq c e^{\left(-\varepsilon / \varepsilon_{0}\right)|x-y|} .
$$

For any $t \in(0, \tau], x \in \mathbb{R}^{d}$ we have

$$
\int_{\mathbb{R}^{d}}\left|q_{0}(t, x, y)\right| d y \leq c t^{-1 / 2} .
$$

For any $t \in(0, \tau], y \in \mathbb{R}^{d}$ we have

$$
\int_{\mathbb{R}^{d}}\left|q_{0}(t, y, x)\right| d x \leq c t^{-1 / 2} .
$$

Proof We have

$q_{0}(t, x, y)=\sum_{i=1}^{d} \lim _{\zeta \rightarrow 0^{+}} \int_{|w|>\zeta}\left[p_{y}\left(t, x-y+a_{i}(x) w\right)-p_{y}\left(t, x-y+a_{i}(y) w\right)\right] \mu(w) d w$.

For $i=1, \ldots, d$ we put

$$
R_{i}=\lim _{\zeta \rightarrow 0^{+}} \int_{|w|>\zeta}\left[p_{y}\left(t, x-y+a_{i}(x) w\right)-p_{y}\left(t, x-y+a_{i}(y) w\right)\right] \mu(w) d w .
$$

We have $q_{0}(t, x, y)=R_{1}+\ldots+R_{d}$. It is clear that it is enough to handle $R_{1}$ alone. Note that

$$
\begin{aligned}
R_{1}=\operatorname{det}(B(y)) \lim _{\zeta \rightarrow 0^{+}} \int_{|w|>\zeta} & {\left[G_{t}\left(\left(x-y+w e_{1}(A(x))^{T}\right)(B(y))^{T}\right)\right.} \\
& \left.-G_{t}\left(\left(x-y+w e_{1}(A(y))^{T}\right)(B(y))^{T}\right)\right] \mu(w) d w .
\end{aligned}
$$

We will use the following abbreviations

$$
\begin{aligned}
& z_{i}=B_{i}(x, y)=b_{i}(y) \cdot(x-y)=b_{i 1}(y)\left(x_{1}-y_{1}\right)+\ldots+b_{i d}(y)\left(x_{d}-y_{d}\right), \\
& k_{i}=\tilde{b}_{i 1}(x, y)=b_{i}(y) \cdot a_{1}(x), \\
& k_{i 0}=\tilde{b}_{i 1}(x, x) .
\end{aligned}
$$

Note that $k_{10}=1$ and $k_{i 0}=0,2 \leq i \leq d$.

We can rewrite (38) as

$$
R_{1}=\operatorname{det}(B(y)) \lim _{\zeta \rightarrow 0^{+}} \int_{|w|>\zeta}\left[\prod_{i=1}^{d} g_{t}\left(z_{i}+k_{i} w\right)-\prod_{i=1}^{d} g_{t}\left(z_{i}+k_{i 0} w\right)\right] \mu(w) d w .
$$


Hence,

$$
R_{1}=\operatorname{det}(B(y))\left(\mathrm{I}_{1}+\ldots+\mathrm{I}_{d}\right)=\operatorname{det}(B(y))\left(\mathrm{I}_{1}^{\prime}+\ldots+\mathrm{I}_{d}^{\prime}\right),
$$

where

$$
\begin{aligned}
\mathrm{I}_{l}= & \int_{\mathbb{R}}\left(\prod_{i=1}^{l-1} g_{t}\left(z_{i}+k_{i} w\right)\right)\left[g_{t}\left(z_{l}+k_{l} w\right)-g_{t}\left(z_{l}+k_{l 0} w\right)\right]\left(\prod_{i=l+1}^{d} g_{t}\left(z_{i}\right)\right) \mu(w) d w, \\
\mathrm{I}_{l}^{\prime}= & \int_{\mathbb{R}}\left(\prod_{i=1}^{l-1} g_{t}\left(z_{i}+k_{i 0} w\right)\right)\left[g_{t}\left(z_{l}+k_{l} w\right)-g_{t}\left(z_{l}+k_{l 0} w\right)\right] \\
& \times\left(\prod_{i=l+1}^{d} g_{t}\left(z_{i}+k_{i} w\right)\right) \mu(w) d w
\end{aligned}
$$

for $l=1, \ldots, d$ (with convention that $\prod_{i=1}^{0}=1=\prod_{i=d+1}^{d}$ ).

We start with the proof of the bound of $q_{0}$. We observe that $\left|k_{l}\right|,\left|k_{l 0}\right| \leq d \eta_{1}^{2} \leq \varepsilon /(4 \delta)$, $l \in\{1, \ldots, d\}$. By Lemmas 2.1 and 2.4, we obtain for $l \leq d-1$,

$$
\begin{aligned}
\left|\mathrm{I}_{l}\right| & \leq g_{t}\left(z_{d}\right) g_{t}^{d-2}(0) \int_{\mathbb{R}}\left|g_{t}\left(z_{l}+k_{l} w\right)-g_{t}\left(z_{l}+k_{l 0} w\right)\right| \mu(w) d w \\
& \leq c\left(\left|k_{l}\right|+\left|k_{l 0}\right|\right)^{\alpha} g_{t}\left(z_{d}\right) g_{t}^{d-2}(0) \frac{h_{t}(0)}{t} \\
& \leq c t^{-1-(d-1) / \alpha} h_{t}\left(z_{d}\right) .
\end{aligned}
$$

The same argument leads to

$$
\begin{aligned}
\left|\mathrm{I}_{d}\right| & \leq g_{t}^{d-1}(0) \int_{\mathbb{R}}\left|g_{t}\left(z_{d}+k_{d} w\right)-g_{t}\left(z_{d}\right)\right| \mu(w) d w \\
& \leq c g_{t}^{d-1}(0)\left|k_{d}\right|^{\alpha} \frac{h_{t}\left(z_{d} / 2\right)}{t} \\
& \leq c t^{-1-(d-1) / \alpha} h_{t}\left(z_{d} / 2\right) .
\end{aligned}
$$

The above inequalities yield

$$
\left|R_{1}\right| \leq c t^{-1-(d-1) / \alpha} h_{t}\left(z_{d} / 2\right) .
$$

Since $R_{1}$ is invariant with respect to permutations of $z_{2}, \ldots, z_{d}$ we infer that

$$
\left|R_{1}\right| \leq c t^{-1-(d-1) / \alpha} \inf _{2 \leq i \leq d} h_{t}\left(z_{i} / 2\right)=c t^{-1-(d-1) / \alpha} h_{t}\left(\max _{2 \leq i \leq d}\left|z_{i}\right| / 2\right) .
$$

On the other hand, again by Lemma 2.4,

$$
\begin{aligned}
\left|\mathrm{I}_{1}^{\prime}\right| & \leq g_{t}^{d-1}(0) \int_{\mathbb{R}}\left|g_{t}\left(z_{1}+k_{1} w\right)-g_{t}\left(z_{1}+w\right)\right| \mu(w) d w \\
& \leq c\left(\left|k_{1}\right|+1\right)^{\alpha} g_{t}^{d-1}(0) \frac{h_{t}\left(z_{1} / 2\right)}{t} \\
& \leq c t^{-1-(d-1) / \alpha} h_{t}\left(z_{1} / 2\right) .
\end{aligned}
$$


For $l \geq 2$, a similar argument leads to

$$
\begin{aligned}
\left|\mathrm{I}_{l}^{\prime}\right| & \leq \sup _{|w| \leq 2 \delta} g_{t}\left(z_{1}+w\right) g_{t}^{d-2}(0) \int_{\mathbb{R}}\left|g_{t}\left(z_{l}+k_{l} w\right)-g_{t}\left(z_{l}\right)\right| \mu(w) d w \\
& \leq c \sup _{|w| \leq 2 \delta} g_{t}\left(z_{1}+w\right) g_{t}^{d-2}(0) \frac{h_{t}(0)}{t} \\
& \leq c t^{-1-(d-1) / \alpha} \sup _{|w| \leq 2 \delta} g_{t}\left(z_{1}+w\right) .
\end{aligned}
$$

Observing that $\left|z_{1}+w\right| \geq\left|z_{1}\right| / 2$ for $|w| \leq 2 \delta \leq 4 \delta \leq\left|z_{1}\right|$ we conclude that

$$
\left|R_{1}\right| \leq c t^{-1-(d-1) / \alpha} h_{t}\left(\frac{\left|z_{1}\right|}{2} \mathbf{1}_{[4 \delta, \infty)}\left(\left|z_{1}\right|\right)\right) .
$$

Combining (39) and (40) we arrive at

$$
\left|R_{1}\right| \leq c t^{-1-(d-1) / \alpha} h_{t}\left(\frac{\max _{1 \leq i \leq d}\left|z_{i}\right|}{2} \mathbf{1}_{[4 \delta, \infty)}\left(\max _{1 \leq i \leq d}\left|z_{i}\right|\right)\right) .
$$

By Lemma 3.2, $\max _{1 \leq i \leq d}\left|z_{i}\right| \geq \frac{1}{\eta_{1} d^{3 / 2}}|x-y|$, and by the choice of $\varepsilon, \varepsilon_{0}, \delta$ we have $\frac{1}{2 \eta_{1} d^{3 / 2}} \geq \varepsilon / \varepsilon_{0}$ and $4 \delta \eta_{1} d^{3 / 2} \leq \varepsilon_{0}$, hence

$$
\begin{aligned}
\left|R_{1}\right| & \leq c t^{-1-(d-1) / \alpha} h_{t}\left(\frac{|x-y|}{2 \eta_{1} d^{3 / 2}} \mathbf{1}_{\left[4 \delta \eta_{1} d^{3 / 2}, \infty\right)}(|x-y|)\right) \\
& \leq c t^{-1-(d-1) / \alpha} h_{t}\left(\left(\varepsilon / \varepsilon_{0}\right)|x-y| \mathbf{1}_{\left[\varepsilon_{0}, \infty\right)}(|x-y|)\right) .
\end{aligned}
$$

Finally, for $|y-x| \geq \varepsilon_{0}$ we have

$$
\left|R_{1}\right| \leq c e^{-\left(\varepsilon / \varepsilon_{0}\right)|x-y|} .
$$

Next, we prove the bound of the integral (35). Let $x \in \mathbb{R}^{d}$ be fixed. Applying Corollary 3.8 with $b_{i}^{*}=k_{i} \mathbf{1}_{\{i \leq l\}}$ we have that

$$
\int_{|y-x| \leq \varepsilon_{0}}\left|\mathrm{I}_{l}\right| d y \leq c t^{-1 / 2}
$$

Hence,

$$
\int_{|x-y| \leq \varepsilon_{0}}\left|q_{0}(t, x, y)\right| d y \leq c t^{-1 / 2} .
$$

For $|y-x| \geq \varepsilon_{0}$ we have $\left|q_{0}(t, x, y)\right| \leq c e^{-\left(\varepsilon / \varepsilon_{0}\right)|x-y|}$ which implies that

$$
\int_{|x-y| \geq \varepsilon_{0}}\left|q_{0}(t, x, y)\right| d y \leq c .
$$

This completes the proof of (35). The estimate (36) is proved exactly in the same way.

Using similar arguments as in the proof of Proposition 3.9 we obtain the following result.

Proposition 3.10 For any $t \in(0, \tau], x \in \mathbb{R}^{d}$ we have

$$
\begin{gathered}
\int_{\mathbb{R}^{d}} p_{y}(t, x-y) d y \leq c, \\
\int_{\mathbb{R}^{d}} r_{y}(t,(x-y) / 2) d y \leq c .
\end{gathered}
$$


For any $\delta_{1}>0$,

$$
\lim _{t \rightarrow 0^{+}} \sup _{x \in \mathbb{R}^{d}} \int_{B^{c}\left(x, \delta_{1}\right)} p_{y}(t, x-y) d y=0 .
$$

We have

$$
\lim _{t \rightarrow 0^{+}} \int_{\mathbb{R}^{d}} p_{y}(t, x-y) d y=1,
$$

uniformly with respect to $x \in \mathbb{R}^{d}$.

Proof For fixed $x \in \mathbb{R}^{d}$ we introduce new variables $u=\tilde{\Psi}_{x}(y)$ given by

$$
u=(x-y) B(y)^{T} \text {. }
$$

Note that

$$
\frac{1}{d \eta_{1}}|x-y| \leq|u|=\left|(x-y) B(y)^{T}\right| \leq d \eta_{1}|x-y| .
$$

For $r>0$, let $V_{x}(r)$ be the $\tilde{\Psi}_{x}$ image of the ball $B(x, r)$. By Remark 3.7 we have almost surely

$$
\left|J_{\tilde{\Psi}_{x}}(y)\right| \geq(1 / 2)|\operatorname{det} B(y)| \geq c, \quad|y-x| \leq \varepsilon_{0},
$$

and $\tilde{\Psi}_{x}$ is an injective map on $B\left(x, \varepsilon_{0}\right)$. Hence, for $0<\delta_{1}<\varepsilon_{0}$, by the change of variables formula (see e.g. [14, Theorem 3]), and then by (45) we obtain

$$
\begin{aligned}
\int_{\delta_{1} \leq|x-y| \leq \varepsilon_{0}} r_{y}(t,(x-y) / 2) d y & \leq c \int_{\delta_{1} \leq|x-y| \leq \varepsilon_{0}} H_{t}(u / 2)\left|J_{\tilde{\Psi}_{x}}(y)\right| d y \\
& =c \int_{V_{x}\left(\varepsilon_{0}\right) \backslash V_{x}\left(\delta_{1}\right)} H_{t}(u / 2) d u \\
& \leq c \int_{|u| \geq \frac{\delta_{1}}{2 d \eta_{1}}} H_{t}(u / 2) d u=I\left(t, \delta_{1}\right) .
\end{aligned}
$$

It is clear that

$$
\lim _{\delta_{1} \rightarrow 0^{+}} I\left(t, \delta_{1}\right) \leq c, t \leq \tau .
$$

If $|x-y| \geq \varepsilon_{0}$ then $|x-y| / 2 \geq \varepsilon \eta_{1} d^{3 / 2}$, hence, by (27), we obtain

$$
\int_{|x-y| \geq \varepsilon_{0}} r_{y}(t,(x-y) / 2) d y \leq c_{1} t \int_{|x-y| \geq \varepsilon_{0}} e^{-c|x-y|} d y=c_{2} t .
$$

The last two inequalities prove that

$$
\sup _{x \in \mathbb{R}^{d}} \sup _{t \leq \tau} \int_{\mathbb{R}^{d}} r_{y}(t,(x-y) / 2) d y<\infty .
$$

Noting that $\lim _{t \rightarrow 0^{+}} I\left(t, \delta_{1}\right)=0$ we obtain

$$
\lim _{t \rightarrow 0^{+}} \sup _{x \in \mathbb{R}^{d}} \int_{|x-y| \geq \delta_{1}} r_{y}(t,(x-y) / 2) d y=0 .
$$

Since $p_{y}(t, x-y) \leq c r_{y}(t,(x-y) / 2)$ for $t \leq \tau, x, y \in \mathbb{R}^{d}$, the proof of $(41,42,43)$ is completed.

Note that the coordinates of the matrix $B(y)$ have partial derivatives $y$ almost surely, bounded uniformly. We can calculate the absolute value of the Jacobian determinant $J_{\tilde{\Psi}_{x}}(y)$, $y$ almost surely, as

$$
\left|J_{\tilde{\Psi}_{x}}(y)\right|=\operatorname{det} B(y)+R(x, y), \quad|R(x, y)| \leq c|y-x| .
$$


Next,

$$
\begin{aligned}
\int_{|x-y| \leq \delta_{1}} p_{y}(t, x-y) d y & =\int_{|x-y| \leq \delta_{1}} G_{t}(u) \operatorname{det} B(y) d y \\
& =\int_{|x-y| \leq \delta_{1}} G_{t}(u)\left|J_{\tilde{\Psi}_{x}}(y)\right| d y-\int_{|x-y| \leq \delta_{1}} G_{t}(u) R(x, y) d y \\
& =I_{1}+I_{2} .
\end{aligned}
$$

Applying (46), (45) and the change of variable formula we obtain

$$
\begin{aligned}
\left|I_{2}\right| & \leq c \int_{|x-y| \leq \delta_{1}}|x-y| G_{t}(u) d y \\
& \leq c \int_{|x-y| \leq \delta_{1}}|u| G_{t}(u)\left|J_{\tilde{\Psi}_{x}}(y)\right| d y \\
& =c \int_{V_{x}\left(\delta_{1}\right)}|u| G_{t}(u) d u \\
& \leq c \int_{|u| \leq d \eta_{1} \delta_{1}}|u| G_{t}(u) d u \rightarrow 0, \text { if } t \rightarrow 0^{+} .
\end{aligned}
$$

Now we can pick, independenly of $x$, positive $\delta_{1}$ and $\delta_{2}$ such that $B\left(0, \delta_{2}\right) \subset V_{x}\left(\delta_{1}\right)$ (see Remark 3.7). Applying again the change of variable formula we obtain

$$
I_{1}=\int_{V_{x}\left(\delta_{1}\right)} G_{t}(u) d u \geq \int_{|u| \leq \delta_{2}} G_{t}(u) d u \rightarrow 1 \text {, if } t \rightarrow 0^{+} .
$$

This completes the proof that uniformly with respect to $x$,

$$
\lim _{t \rightarrow 0^{+}} \int_{|x-y| \leq \delta_{1}} p_{y}(t, x-y) d y=1,
$$

which combined with (43) proves (44).

In the sequel we will use the following standard estimate. For any $\gamma \in(0,1], \theta_{0}>0$ there exists $c=c\left(\gamma, \theta_{0}\right)$ such that for any $\theta \geq \theta_{0}, t>0$ we have

$$
\int_{0}^{t}(t-s)^{\gamma-1} s^{\theta-1} d s \leq \frac{c}{\theta^{\gamma}} t^{(\gamma-1)+(\theta-1)+1} .
$$

Lemma 3.11 For any $t>0, x \in \mathbb{R}^{d}$ and $n \in \mathbb{N}$ the kernel $q_{n}(t, x, y)$ is well defined. For any $t \in(0, \tau], x \in \mathbb{R}^{d}$ and $n \in \mathbb{N}$ we have

$$
\begin{gathered}
\int_{\mathbb{R}^{d}}\left|q_{n}(t, x, y)\right| d y \leq \frac{c_{1}^{n+1} t^{(n+1) / 2-1}}{(n !)^{1 / 2}}, \\
\int_{\mathbb{R}^{d}}\left|q_{n}(t, y, x)\right| d y \leq \frac{c_{1}^{n+1} t^{(n+1) / 2-1}}{(n !)^{1 / 2}} .
\end{gathered}
$$

For any $t \in(0, \tau], x, y \in \mathbb{R}^{d}$ and $n \in \mathbb{N}$ we have

$$
\left|q_{n}(t, x, y)\right| \leq c_{1} \frac{c_{2}^{n} t^{n / 2-1}}{(n !)^{1 / 2} t^{d / \alpha}} .
$$


For any $t \in(0, \tau], x, y \in \mathbb{R}^{d}$ and $n \in \mathbb{N},|x-y| \geq n+1$ we have

$$
\left|q_{n}(t, x, y)\right| \leq c_{1} \frac{c_{2}^{n} t^{n / 2}}{(n !)^{1 / 2}} e^{-\frac{\lambda|x-y|}{n+1}}
$$

where $\lambda=\varepsilon / \varepsilon_{0}$.

Proof By Proposition 3.9, there is a constant $c^{*}$ such that for any $x, y \in \mathbb{R}^{d}, t \in(0, \tau]$ we have

$$
\begin{gathered}
\left|q_{0}(t, x, y)\right| \leq c^{*} \frac{1}{t^{1+d / \alpha}}, \\
\left|q_{0}(t, x, y)\right| \leq c^{*} e^{-\lambda|x-y|},|x-y| \geq 1 . \\
\int_{\mathbb{R}^{d}}\left|q_{0}(t, x, u)\right| d u \leq c^{*} t^{-1 / 2}, \\
\int_{\mathbb{R}^{d}}\left|q_{0}(t, u, x)\right| d u \leq c^{*} t^{-1 / 2} .
\end{gathered}
$$

It follows from (47) there is $p \geq 1$ such that for $n \in \mathbb{N}$,

$$
\begin{gathered}
\int_{0}^{t}(t-s)^{-1 / 2} s^{n / 2-1 / 2} d s \leq \frac{p}{(n+1)^{1 / 2}} t^{n / 2}, \\
\int_{t / 2}^{t}(t-s)^{-1 / 2} s^{n / 2-1} d s \leq \frac{p}{(n+1)^{1 / 2}} t^{(n+1) / 2-1}, \\
\int_{0}^{t}(t-s)^{-1 / 2} s^{n / 2} d s \leq \frac{p}{(n+1)^{1 / 2}} t^{(n+1) / 2} .
\end{gathered}
$$

We define $c_{1}=p c^{*} \geq c^{*}$ and $c_{2}=2^{d / \alpha+1} c_{1}(2+p)>c_{1}$.

We will prove (48), (49), (50) simultaneously by induction. They are true for $n=0$ by $(52,54,55)$ and the choice of $c_{1}$. Assume that (48), (49), (50) are true for $n \in \mathbb{N}$, we will show them for $n+1$. By the definition of $q_{n}(t, x, y)$ and the induction hypothesis we obtain

$$
\begin{aligned}
\left|q_{n+1}(t, x, y)\right| \leq & c_{1} \frac{2^{d / \alpha+1}}{t^{d / \alpha+1}} \int_{0}^{t / 2} \int_{\mathbb{R}^{d}}\left|q_{n}(s, z, y)\right| d z d s \\
& +c_{1} \frac{c_{2}^{n} 2^{d / \alpha+1}}{(n !)^{1 / 2} t^{d / \alpha}} \int_{t / 2}^{t} \int_{\mathbb{R}^{d}}\left|q_{0}(t-s, x, z)\right| d z s^{n / 2-1} d s \\
\leq & c_{1} \frac{c_{1}^{n+1} 2^{d / \alpha+1}}{(n !)^{1 / 2} t^{d / \alpha+1}} \int_{0}^{t / 2} s^{(n+1) / 2-1} d s \\
& +c_{1} \frac{c_{2}^{n} 2^{d / \alpha+1} c_{1}}{(n !)^{1 / 2} t^{d / \alpha}} \int_{t / 2}^{t}(t-s)^{-1 / 2} s^{n / 2-1} d s \\
\leq & c_{1} \frac{c_{2}^{n} t^{(n+1) / 2}}{((n+1) !)^{1 / 2} t^{d / \alpha+1}}\left(2 c_{1} 2^{d / \alpha+1}+c_{1} 2^{d / \alpha+1} p\right) \\
= & c_{1} \frac{c_{2}^{n+1} t^{(n+1) / 2}}{((n+1) !)^{1 / 2} t^{d / \alpha+1}} .
\end{aligned}
$$

Hence we get (50) for $n+1$. In particular this gives that the kernel $q_{n+1}(t, x, y)$ is well defined. 
By the definition of $q_{n}(t, x, y),(54)$ and the induction hypothesis we obtain

$$
\begin{aligned}
\int_{\mathbb{R}^{d}}\left|q_{n+1}(t, x, y)\right| d y & \leq \int_{0}^{t} \int_{\mathbb{R}^{d}} \int_{\mathbb{R}^{d}}\left|q_{0}(t-s, x, z)\right|\left|q_{n}(s, z, y)\right| d z d y d s \\
& \leq c^{*} \frac{c_{1}^{n+1}}{(n !)^{1 / 2}} \int_{0}^{t}(t-s)^{-1 / 2} s^{(n+1) / 2-1} d s \\
& \leq c^{*} \frac{c_{1}^{n+1}}{(n !)^{1 / 2}} \frac{p}{(n+1)^{1 / 2}} t^{n / 2} \\
& =\frac{c_{1}^{n+2}}{((n+1) !)^{1 / 2}} t^{n / 2}
\end{aligned}
$$

which proves (48) for $n+1$. Similarly we get (49).

Now we will show (51). For $n=0$ this follows from (53). Assume that (51) is true for $n \in \mathbb{N}$, we will show it for $n+1$.

Using our induction hypothesis, (48) and (49) we get for $|x-y| \geq n+2$,

$$
\begin{aligned}
\left|q_{n+1}(t, x, y)\right|= & \mid \int_{0}^{t} \int_{|x-z| \geq \frac{|x-y|}{n+2}} q_{0}(t-s, x, z) q_{n}(s, z, y) d z d s \\
& +\int_{0}^{t} \int_{|x-z| \leq \frac{|x-y|}{n+2}} q_{0}(t-s, x, z) q_{n}(s, z, y) d z d s \mid \\
\leq & c_{1} e^{-\frac{\lambda|x-y|}{n+2}} \int_{0}^{t} \int_{\mathbb{R}^{d}}\left|q_{n}(s, z, y)\right| d z d s \\
& +c_{1} \frac{c_{2}^{n}}{(n !)^{1 / 2}} e^{-\frac{\lambda|x-y|}{n+2}} \int_{0}^{t} \int_{\mathbb{R}^{d}}\left|q_{0}(t-s, x, z)\right| d z s^{n / 2} d s \\
\leq & c_{1} \frac{2 c_{1}^{n+1} t^{(n+1) / 2}}{((n+1) !)^{1 / 2}} e^{-\frac{\lambda|x-y|}{n+2}}+c_{1} \frac{c_{2}^{n} c^{*} t^{(n+1) / 2} p}{((n+1) !)^{1 / 2}} e^{-\frac{\lambda|x-y|}{n+2}} \\
= & \left(2 c_{1} c_{1}^{n+1}+c_{2}^{n} c_{1}^{2}\right) \frac{t^{(n+1) / 2}}{((n+1) !)^{1 / 2}} e^{-\frac{\lambda|x-y|}{n+2}},
\end{aligned}
$$

which proves (51) for $n+1$ since by the choice of constants $2 c_{1} c_{1}^{n+1}+c_{2}^{n} c_{1}^{2} \leq c_{1} c_{2}^{n+1}$.

By standard estimates, one easily gets

$$
\sum_{n=k}^{\infty} \frac{C^{n}}{(n !)^{1 / 2}} \leq \frac{C^{k}}{(k !)^{1 / 2}} \sum_{n=k}^{\infty} \frac{C^{n-k}}{((n-k) !)^{1 / 2}} \leq C_{1} e^{-k}, \quad k \in \mathbb{N},
$$

where $C_{1}$ depends on $C$.

Proposition 3.12 For any $t \in(0, \infty), x, y \in \mathbb{R}^{d}$ the kernel $q(t, x, y)$ is well defined. For any $t \in(0, \tau], x, y \in \mathbb{R}^{d}$ we have

$$
|q(t, x, y)| \leq \frac{c}{t^{d / \alpha+1}} e^{-c_{3} \sqrt{|x-y|}} \leq \frac{c}{t^{d / \alpha+1}(1+|x-y|)^{d+1}} .
$$

There exists $a>0$ (a depends on $\left.\tau, \alpha, d, \eta_{1}, \eta_{2}, \eta_{3}\right)$ such that for any $t \in(0, \tau], x, y \in \mathbb{R}^{d}$, $|x-y| \geq a$ we have

$$
|q(t, x, y)| \leq c e^{-c_{3} \sqrt{|x-y|}} .
$$


For any $t \in(0, \tau]$ and $x \in \mathbb{R}^{d}$ we have

$$
\begin{aligned}
& \int_{\mathbb{R}^{d}}|q(t, x, y)| d y \leq c t^{-1 / 2}, \\
& \int_{\mathbb{R}^{d}}|q(t, y, x)| d y \leq c t^{-1 / 2} .
\end{aligned}
$$

Proof By (50), we clearly get $\sum_{n=0}^{\infty}\left|q_{n}(t, x, y)\right| \leq c t^{-d / \alpha-1}$. This gives that $q(t, x, y)$ is well defined and we have $|q(t, x, y)| \leq c t^{-d / \alpha-1}$.

For $|x-y| \geq 1$ by (50), (51) and (56) we get

$$
\begin{aligned}
|q(t, x, y)| & =\left|\sum_{n=0}^{[\sqrt{|x-y|}-1]} q_{n}(t, x, y)+\sum_{n=[\sqrt{|x-y|}]}^{\infty} q_{n}(t, x, y)\right| \\
& \leq c_{1} \sum_{n=0}^{[\sqrt{|x-y|}-1]} \frac{c_{2}^{n} \tau^{n / 2}}{(n !)^{1 / 2}} e^{-\lambda \sqrt{|x-y|}}+c_{1} \sum_{n=[\sqrt{|x-y|}]}^{\infty} \frac{c_{2}^{n} \tau^{n / 2}}{(n !)^{1 / 2} t^{d / \alpha+1}} \\
& \leq \frac{c}{t^{d / \alpha+1}} e^{-c_{3} \sqrt{|x-y|}},
\end{aligned}
$$

where $[z]$ denotes the integer part of $[z]$. Take the smallest $n_{0} \in \mathbb{N}$ such that $n_{0} / 2-1 \geq d / \alpha$. For $\sqrt{|x-y|} \geq n_{0}$ we get

$$
\begin{aligned}
|q(t, x, y)| & \leq c_{1} \sum_{n=0}^{[\sqrt{|x-y|}-1]} \frac{c_{2}^{n} \tau^{n / 2}}{(n !)^{1 / 2}} e^{-\lambda \sqrt{|x-y|}}+c_{1} \sum_{n=[\sqrt{|x-y|}]}^{\infty} \frac{c_{2}^{n} t^{n / 2}}{(n !)^{1 / 2} t^{d / \alpha+1}} \\
& \leq c e^{-c_{3} \sqrt{|x-y|}} .
\end{aligned}
$$

The estimates (57) and (58) follow easily from (48) and (49).

By (25), Corollary 3.3, Proposition 3.10 and Proposition 3.12, we immediately obtain the following result.

Corollary 3.13 For any $t \in(0, \infty), x, y \in \mathbb{R}^{d}$ the kernel $u(t, x, y)$ is well defined. For any $t \in(0, \tau], x, y \in \mathbb{R}^{d}$ we have

$$
|u(t, x, y)| \leq \frac{c}{t^{d / \alpha}} e^{-c_{1} \sqrt{|x-y|}} \leq \frac{c}{t^{d / \alpha}(1+|x-y|)^{d+1}} .
$$

There exists $a>\varepsilon>0$ (a depends on $\tau, \alpha, d, \eta_{1}, \eta_{2}, \eta_{3}$ ) such that for any $t \in(0, \tau]$, $x, y \in \mathbb{R}^{d},|x-y| \geq a$ we have

$$
|u(t, x, y)| \leq c e^{-c_{2} \sqrt{|x-y|}} .
$$

For any $t \in(0, \tau]$ and $x \in \mathbb{R}^{d}$ we have

$$
\begin{gathered}
\int_{\mathbb{R}^{d}}|u(t, x, y)| d y \leq c, \\
\int_{\mathbb{R}^{d}}|u(t, y, x)| d y \leq c .
\end{gathered}
$$


Proof By Corollary 3.3, Proposition 3.10, (25), we only need to prove the corresponding bounds for

$$
I(t, x, y)=\int_{0}^{t} \int_{\mathbb{R}^{d}} p_{z}(t-s, x-z)|q(s, z, y)| d z d s .
$$

For $0<s<t / 2$ we have

$$
p_{z}(t-s, x-z) \leq \frac{c}{t^{d / \alpha}},
$$

and, by Proposition 3.12, for $t / 2<s<t$,

$$
|q(s, z, y)| \leq \frac{c}{t^{d / \alpha+1}} .
$$

Hence,

$$
\begin{aligned}
I(t, x, y)= & \int_{0}^{t / 2} \int_{\mathbb{R}^{d}} p_{z}(t-s, x-z)|q(s, z, y)| d z d s \\
& +\int_{t / 2}^{t} \int_{\mathbb{R}^{d}} p_{z}(t-s, x-z)|q(s, z, y)| d z d s \\
\leq & \frac{c}{t^{d / \alpha}} \int_{0}^{t / 2} \int_{\mathbb{R}^{d}}|q(s, z, y)| d z d s+\frac{c}{t^{d / \alpha+1}} \int_{t / 2}^{t} \int_{\mathbb{R}^{d}} p_{z}(t-s, x-z) d z d s \\
\leq & \frac{c}{t^{d / \alpha}},
\end{aligned}
$$

where (58) and Proposition 3.10 were applied to estimate the integrals with respect to the space variable.

Let $a$ the constant found in Proposition 3.12. Assume that $|x-y| \geq 1+a$. By Corollary 3.3 , for $0<s<t$, we have

$$
p_{z}(t-s, x-z) \leq c e^{-c_{1}|x-y|}, \quad|x-z|>|x-y|>1 .
$$

Proposition 3.12 implies that for $0<s<t$,

$$
|q(s, z, y)| \leq c e^{-c_{1} \sqrt{|x-y|}}, \quad|y-z|>|x-y|>a
$$

Hence,

$$
\begin{aligned}
I(t, x, y) \leq & \int_{0}^{t} \int_{|x-z|>|x-y|} \ldots d z d s+\int_{0}^{t} \int_{|y-z|>|x-y|} \ldots d z d s \\
\leq & c e^{-c_{1}|x-y|} \int_{0}^{t} \int_{\mathbb{R}^{d}}|q(s, z, y)| d z d s \\
& +c e^{-c_{1} \sqrt{|x-y|}} \int_{0}^{t} \int_{\mathbb{R}^{d}} p_{z}(t-s, x-z) d z d s \\
\leq & c t^{1 / 2} e^{-c_{1}|x-y|}+c t e^{-c_{1} \sqrt{|x-y|}} \\
\leq & c e^{-c_{1} \sqrt{|x-y|}} .
\end{aligned}
$$

Combining (61) and (62) we obtain the desired pointwise estimates of $u(t, x, y)$. Next, (59) and (60) immediately follow from (57), (58) and Proposition 3.10. 
For any $\zeta>0$ and $x, y \in \mathbb{R}^{d}$ we put

$$
\begin{aligned}
& \mathcal{L}_{\zeta} f(x)=\sum_{i=1}^{d} \int_{|w|>\zeta}\left[f\left(x+a_{i}(x) w\right)-f(x)\right] \mu(w) d w, \\
& \mathcal{L}_{\zeta}^{y} f(x)=\sum_{i=1}^{d} \int_{|w|>\zeta}\left[f\left(x+a_{i}(y) w\right)-f(x)\right] \mu(w) d w .
\end{aligned}
$$

Lemma 3.14 For any $\xi \in(0,1], \zeta>0, x, y, v \in \mathbb{R}^{d}$ and $t \in(\xi, \tau+\xi]$ we have

$$
\begin{gathered}
\sum_{i=1}^{d} \int_{\mathbb{R}}\left|p_{y}\left(t, x-y+a_{i}(v) w\right)-p_{y}(t, x-y)\right| \mu(w) d w \leq c(\xi) e^{-c|x-y|}, \\
\sum_{i=1}^{d} \int_{|w| \leq \zeta}\left|p_{y}\left(t, x-y+a_{i}(v) w\right)-p_{y}(t, x-y)\right| \mu(w) d w \leq c(\xi) \zeta^{1-\alpha},
\end{gathered}
$$

where $c(\xi)$ is a constant depending on $\xi, \tau, \alpha, d, \eta_{1}, \eta_{2}, \eta_{3}, \varepsilon$.

Proof We estimate the term for $i=1$. By Lemma 3.1, for $\gamma=1$, we get for $w \in \mathbb{R}$

$$
\begin{aligned}
& \left|p_{y}\left(t, x-y+a_{1}(v) w\right)-p_{y}(t, x-y)\right| \\
& \leq c t^{-1 / \alpha}|w|\left(r_{y}\left(t, \frac{x-y}{2}\right)+r_{y}\left(t, \frac{x-y+a_{1}(v) w}{2}\right)\right) .
\end{aligned}
$$

Recall that, if $|w| \geq 2 \delta$, then $\mu(w)=0$. So we may assume that $|w| \leq 2 \delta$. By Corollary 3.3 , we get

$$
r_{y}\left(t, \frac{x-y}{2}\right)+r_{y}\left(t, \frac{x-y+a_{1}(v) w}{2}\right) \leq c_{1} t^{-d / \alpha} e^{-c|x-y|} .
$$

Now (63) and (64) follow by the fact that $\mu(w) \leq c 1_{[-2 \delta, 2 \delta]}(w)|w|^{-1-\alpha}$.

Lemma 3.15 Let $\tau_{2}>\tau_{1}>0$. Assume that a function $f_{t}(x)$ is bounded and uniformly continuous on $\left[\tau_{1}, \tau_{2}\right] \times \mathbb{R}^{d}$. Then

$$
\sup _{t \in\left[\tau_{1}, \tau_{2}\right], x \in \mathbb{R}^{d}}\left|\int_{\mathbb{R}^{d}} p_{y}\left(\varepsilon_{1}, x-y\right) f_{t}(y) d y-f_{t}(x)\right| \rightarrow 0 \quad \text { as } \quad \varepsilon_{1} \rightarrow 0^{+} .
$$

Proof The lemma follows easily by Propostion 3.10.

For any $t>0, x, y \in \mathbb{R}^{d}$ we define

$$
\varphi_{y}(t, x)=\int_{0}^{t} \int_{\mathbb{R}^{d}} p_{z}(t-s, x-z) q(s, z, y) d z d s .
$$

Clearly we have

$$
u(t, x, y)=p_{y}(t, x-y)+\varphi_{y}(t, x)
$$


For any $t>0, x, y \in \mathbb{R}^{d}, f \in \mathcal{B}_{b}\left(\mathbb{R}^{d}\right)$ we define

$$
\begin{aligned}
\Phi_{t} f(x) & =\int_{\mathbb{R}^{d}} \varphi_{y}(t, x) f(y) d y, \\
U_{t} f(x) & =\int_{\mathbb{R}^{d}} u(t, x, y) f(y) d y . \\
Q_{t} f(x) & =\int_{\mathbb{R}^{d}} q(t, x, y) f(y) d y .
\end{aligned}
$$

Now, following ideas from [19], we will define the so-called approximate solutions.

For any $t \geq 0, \xi \in[0,1], t+\xi>0, x, y \in \mathbb{R}^{d}$ we define

$$
\varphi_{y}^{(\xi)}(t, x)=\int_{0}^{t} \int_{\mathbb{R}^{d}} p_{z}(t-s+\xi, x-z) q(s, z, y) d z d s
$$

and

$$
u^{(\xi)}(t, x, y)=p_{y}(t+\xi, x-y)+\varphi_{y}^{(\xi)}(t, x) .
$$

For any $t \geq 0, \xi \in[0,1], t+\xi>0, x, y \in \mathbb{R}^{d}, f \in \mathcal{B}_{b}\left(\mathbb{R}^{d}\right)$ we define

$$
\begin{gathered}
\Phi_{t}^{(\xi)} f(x)=\int_{\mathbb{R}^{d}} \varphi_{y}^{(\xi)}(t, x) f(y) d y, \\
U_{t}^{(\xi)} f(x)=\int_{\mathbb{R}^{d}} u^{(\xi)}(t, x, y) f(y) d y, \\
\Phi_{0} f(x)=0, \quad U_{0}^{(0)} f(x)=U_{0} f(x)=f(x) .
\end{gathered}
$$

By the same arguments as in the proof of Corollary 3.13 we obtain the following result.

Corollary 3.16 For any $t \in[0, \infty), \xi \in[0,1], t+\xi>0, x, y \in \mathbb{R}^{d}$ the kernel $u^{(\xi)}(t, x, y)$ is well defined. For any $t \in(0, \tau], \xi \in[0,1], x, y \in \mathbb{R}^{d}$ we have

$$
\left|u^{(\xi)}(t, x, y)\right| \leq \frac{c}{(t+\xi)^{d / \alpha}(1+|x-y|)^{d+1}} .
$$

For any $t \in(0, \tau], \xi \in[0,1]$ and $x \in \mathbb{R}^{d}$ we have

$$
\begin{aligned}
& \int_{\mathbb{R}^{d}}\left|u^{(\xi)}(t, x, y)\right| d y \leq c, \\
& \int_{\mathbb{R}^{d}}\left|u^{(\xi)}(t, y, x)\right| d y \leq c .
\end{aligned}
$$

Lemma 3.17 Let $f \in C_{0}\left(\mathbb{R}^{d}\right)$ and $0<\tau_{1}<\tau_{2}<\tau$. Then $Q_{t} f(x)$ as a function of $(t, x)$ is uniformly continuous on $\left[\tau_{1}, \tau_{2}\right] \times \mathbb{R}^{d}$. We have $\lim _{|x| \rightarrow \infty} Q_{t} f(x)=0$ uniformly in $t \in\left[\tau_{1}, \tau_{2}\right]$. For each $t>0$ we have $Q_{t} f \in C_{0}\left(\mathbb{R}^{d}\right)$.

Proof For any $\zeta>0, y \in \mathbb{R}^{d}$, by Lemma 3.4, we obtain that

$$
(t, x) \rightarrow \mathcal{L}_{\zeta}^{x} p_{y}(t, \cdot)(x-y)-\mathcal{L}_{\zeta}^{y} p_{y}(t, \cdot)(x-y)
$$

is continuous on $(0, \infty) \times \mathbb{R}^{d}$. Using this and (64) we obtain that

$$
(t, x) \rightarrow q_{0}(t, x, y) \quad \text { is continuous on }(0, \infty) \times \mathbb{R}^{d} .
$$

By Proposition 3.9, we have

$$
\left|q_{0}(t, x, y)\right| \leq \frac{c}{t^{1+d / \alpha}} e^{-c_{1}|x-y|} .
$$


For any $n \in \mathbb{N}, t>0, x \in \mathbb{R}^{d}$ denote

$$
Q_{n, t} f(x)=\int_{\mathbb{R}^{d}} q_{n}(t, x, y) f(y) d y .
$$

By (65), (66) and the dominated convergence theorem, we obtain that $(t, x) \rightarrow Q_{0, t} f(x)$ is continuous on $(0, \infty) \times \mathbb{R}^{d}$. By Lemma 3.11 , for any $t \in(0, \tau], x \in \mathbb{R}^{d}, n \in \mathbb{N}$ we have

$$
\left|Q_{n, t} f(x)\right| \leq \frac{c_{1}^{n+1} t^{(n+1) / 2-1}}{(n !)^{1 / 2}}\|f\|_{\infty} .
$$

Note that for any $t>0, x \in \mathbb{R}^{d}, n \in \mathbb{N}, n \geq 1$ we have

$$
Q_{n, t} f(x)=\int_{0}^{t} \int_{\mathbb{R}^{d}} q_{0}(t-s, x, z) Q_{n-1, s} f(z) d z d s .
$$

For any $\varepsilon_{1} \in\left(0, \tau_{1} / 2\right)$ using (65), (66) and (67) we obtain that

$$
(t, x) \rightarrow \int_{0}^{t-\varepsilon_{1}} \int_{\mathbb{R}^{d}} q_{0}(t-s, x, z) Q_{n-1, s} f(z) d z d s
$$

is continuous on $\left[\tau_{1}, \tau_{2}\right] \times \mathbb{R}^{d}$. Note also that for any $\varepsilon_{1} \in\left(0, \tau_{1} / 2\right), t \in\left[\tau_{1}, \tau_{2}\right], x \in \mathbb{R}^{d}$, $n \in \mathbb{N}, n \geq 1$ we have by (35)

$$
\begin{aligned}
\left|\int_{t-\varepsilon_{1}}^{t} \int_{\mathbb{R}^{d}} q_{0}(t-s, x, z) Q_{n-1, s} f(z) d z d s\right| & \leq c\|f\|_{\infty} \int_{t-\varepsilon_{1}}^{t}(t-s)^{-1 / 2} s^{-1 / 2} d s \\
& \leq c \tau_{1}^{-1 / 2} \varepsilon_{1}^{1 / 2}\|f\|_{\infty} .
\end{aligned}
$$

This implies that $(t, x) \rightarrow Q_{n, t} f(x)$ is continuous on $\left[\tau_{1}, \tau_{2}\right] \times \mathbb{R}^{d}$. Using this and (67) we obtain that $(t, x) \rightarrow Q_{t} f(x)=\sum_{n=0}^{\infty} Q_{n, t} f(x)$ is continuous on $\left[\tau_{1}, \tau_{2}\right] \times \mathbb{R}^{d}$. By Proposition 3.12, we obtain that $\lim _{|x| \rightarrow \infty} Q_{t} f(x)=0$ uniformly in $t \in\left[\tau_{1}, \tau_{2}\right]$. This implies the assertion of the lemma.

Proposition 3.18 Choose $\gamma \in(0, \alpha)$. For any $t \in(0, \tau], x, x^{\prime} \in \mathbb{R}^{d}, f \in \mathcal{B}_{b}\left(\mathbb{R}^{d}\right)$ we have

$$
\left|U_{t} f(x)-U_{t} f\left(x^{\prime}\right)\right| \leq c t^{-\gamma / \alpha}\left|x-x^{\prime}\right|^{\gamma}\|f\|_{\infty} .
$$

Proof We have

$$
\begin{aligned}
U_{t} f(x)-U_{t} f\left(x^{\prime}\right)= & \int_{\mathbb{R}^{d}}\left(p_{y}(t, x-y)-p_{y}\left(t, x^{\prime}-y\right)\right) f(y) d y \\
& +\int_{0}^{t} \int_{\mathbb{R}^{d}}\left(p_{z}(t-s, x-z)-p_{z}\left(t-s, x^{\prime}-z\right)\right) Q_{s} f(z) d z d s \\
= & \mathrm{I}+\mathrm{II} .
\end{aligned}
$$

By Lemma 3.1 and Proposition 3.10, we get

$|\mathrm{I}| \leq c\|f\|_{\infty}\left|x-x^{\prime}\right|^{\gamma} t^{-\gamma / \alpha} \int_{\mathbb{R}^{d}}\left(r_{y}(t,(x-y) / 2)+r_{y}\left(t,\left(x^{\prime}-y\right) / 2\right)\right) d y \leq c\|f\|_{\infty}\left|x-x^{\prime}\right|^{\gamma} t^{-\gamma / \alpha}$. 
By Lemma 3.1 and Propositions 3.10, 3.12, we obtain

$$
\begin{aligned}
|\mathrm{II}| \leq & c\|f\|_{\infty}\left|x-x^{\prime}\right|^{\gamma} \int_{0}^{t} \int_{\mathbb{R}^{d}}(t-s)^{-\gamma / \alpha}\left(r_{z}(t-s,(x-z) / 2)\right. \\
& \left.+r_{z}\left(t-s,\left(x^{\prime}-z\right) / 2\right)\right) s^{-1 / 2} d z d s \\
\leq & c\|f\|_{\infty}\left|x-x^{\prime}\right|^{\gamma} \int_{0}^{t}(t-s)^{-\gamma / \alpha} s^{-1 / 2} d s \\
\leq & c\|f\|_{\infty}\left|x-x^{\prime}\right|^{\gamma} t^{1 / 2-\gamma / \alpha} .
\end{aligned}
$$

Note that, by Lemma 3.14, for any $\xi \in(0,1], t \in[\xi, \tau+\xi], x, z \in \mathbb{R}^{d}$ we have

$$
\left|\frac{\partial p_{z}(t, x-z)}{\partial t}\right|=\left|\mathcal{L}^{z} p_{z}(t, \cdot)(x-z)\right| \leq c(\xi) e^{-c|x-z|},
$$

where $c(\xi)$ is a constant depending on $\xi, \tau, \alpha, d, \eta_{1}, \eta_{2}, \eta_{3}, \varepsilon$.

The next lemma is similar to [19, Lemma 4.1].

Lemma 3.19 (i) For every $f \in C_{0}\left(\mathbb{R}^{d}\right), \xi \in(0,1]$ the function $U_{t}^{(\xi)} f(x)$ belongs to $C^{1}((0, \infty))$ as a function of $t$ and to $C_{0}^{2}\left(\mathbb{R}^{d}\right)$ as a function of $x$. Moreover we have

$$
\left|\frac{\partial}{\partial t}\left(U_{t}^{(\xi)} f\right)(x)\right| \leq c(\xi) t^{-1 / 2}\|f\|_{\infty},
$$

for each $f \in C_{0}\left(\mathbb{R}^{d}\right), t \in(0, \tau], x \in \mathbb{R}^{d}, \xi \in(0,1]$, where $c(\xi)$ depends on $\xi, \tau, \alpha, d, \eta_{1}, \eta_{2}, \eta_{3}$.

(ii) For every $f \in C_{0}\left(\mathbb{R}^{d}\right)$ we have

$$
\lim _{t, \xi \rightarrow 0^{+}}\left\|U_{t}^{(\xi)} f-f\right\|_{\infty}=0 .
$$

(iii) For every $f \in C_{0}\left(\mathbb{R}^{d}\right)$ we have

$$
U_{t}^{(\xi)} f(x) \rightarrow 0, \quad \text { as }|x| \rightarrow \infty,
$$

uniformly in $t \in[0, \tau], \xi \in[0,1]$.

(iv) For every $f \in C_{0}\left(\mathbb{R}^{d}\right)$ we have

$$
\left\|U_{t}^{(\xi)} f-U_{t} f\right\|_{\infty} \rightarrow 0, \quad \text { as } \xi \rightarrow 0^{+},
$$

uniformly in $t \in[0, \tau]$.

Proof (i) Let $f \in C_{0}\left(\mathbb{R}^{d}\right), t \in(0, \tau], \xi \in(0,1]$ and $x \in \mathbb{R}^{d}$. We have

$$
\begin{aligned}
& \lim _{h \rightarrow 0^{+}} \frac{\Phi_{t+h}^{(\xi)} f(x)-\Phi_{t}^{(\xi)} f(x)}{h} \\
= & \lim _{h \rightarrow 0^{+}} \frac{1}{h} \int_{t}^{t+h} \int_{\mathbb{R}^{d}} p_{z}(t+h-s+\xi, x-z) Q_{s} f(z) d z d s \\
& +\lim _{h \rightarrow 0^{+}} \int_{0}^{t} \int_{\mathbb{R}^{d}} \frac{p_{z}(t+h-s+\xi, x-z)-p_{z}(t-s+\xi, x-z)}{h} Q_{s} f(z) d z d s \\
= & \mathrm{I}+\mathrm{II} .
\end{aligned}
$$


By Lemmas 3.4,3.17, Corollary 3.3 and Proposition3.12, we get

$$
\mathrm{I}=\int_{\mathbb{R}^{d}} p_{z}(\xi, x-z) Q_{t} f(z) d z .
$$

By Lemma 3.4, the dominated convergence theorem, (68) and Proposition 3.12, we get

$$
\mathrm{II}=\int_{0}^{t} \int_{\mathbb{R}^{d}} \frac{\partial p_{z}(t-s+\xi, x-z)}{\partial t} Q_{s} f(z) d z d s .
$$

By similar arguments, we get the analogous result for $\lim _{h \rightarrow 0^{-}}$ $\left(\Phi_{t+h}^{(\xi)} f(x)-\Phi_{t}^{(\xi)} f(x)\right) / h$.

By (68), we obtain

$$
\frac{\partial}{\partial t} \int_{\mathbb{R}^{d}} p_{z}(t+\xi, x-z) f(z) d z=\int_{\mathbb{R}^{d}} \frac{\partial}{\partial t} p_{z}(t+\xi, x-z) f(z) d z .
$$

Hence, we have

$$
\begin{aligned}
\frac{\partial}{\partial t}\left(U_{t}^{(\xi)} f\right)(x)= & \int_{\mathbb{R}^{d}} \frac{\partial}{\partial t} p_{z}(t+\xi, x-z) f(z) d z \\
& +\int_{\mathbb{R}^{d}} p_{z}(\xi, x-z) Q_{t} f(z) d z \\
& +\int_{0}^{t} \int_{\mathbb{R}^{d}} \frac{\partial p_{z}(t-s+\xi, x-z)}{\partial t} Q_{s} f(z) d z d s .
\end{aligned}
$$

Using this, (68), Propositions 3.10 and 3.12 we obtain (69). We also obtain that for every $f \in C_{0}\left(\mathbb{R}^{d}\right), \xi \in(0,1]$ the function $U_{t}^{(\xi)} f(x)$ belongs to $C^{1}((0, \infty))$ as a function of $t$.

The fact that $U_{t}^{(\xi)} f \in C_{0}^{2}\left(\mathbb{R}^{d}\right)$ for $\xi \in(0,1]$ follows by Lemmas 3.4, 3.5, Proposition 3.12 and Lemma 3.17.

(ii) Fix $f \in C_{0}\left(\mathbb{R}^{d}\right)$. For any $\xi \in[0,1], t \geq 0, t+\xi>0, x \in \mathbb{R}^{d}$ we have

$$
U_{t}^{(\xi)} f(x)=\int_{\mathbb{R}^{d}} p_{y}(t+\xi, x-y) f(y) d y+\Phi_{t}^{(\xi)} f(x) .
$$

For any $\xi \in[0,1], t \in[0, \tau], \xi+t>0, x \in \mathbb{R}^{d}$, by Propositions 3.12 and 3.10, we get

$$
\left|\Phi_{t}^{(\xi)} f(x)\right| \leq c\|f\|_{\infty} \int_{0}^{t} s^{-1 / 2} d s \leq c\|f\|_{\infty} t^{1 / 2} .
$$

By (43), Proposition 3.10 and the fact that $f$ is uniformly continuous on $\mathbb{R}^{d}$, we obtain

$$
\lim _{t, \xi \rightarrow 0^{+}} \int_{\mathbb{R}^{d}} p_{y}(t+\xi, x-y) f(y) d y-f(x)=0
$$

uniformly with respect to $x \in \mathbb{R}^{d}$. This and (71) gives (ii).

(iii) This follows easily from (ii) and Corollary 3.16.

(iv) Fix $f \in C_{0}\left(\mathbb{R}^{d}\right)$. By Lemma 3.4, Corollary 3.3 and the dominated convergence theorem we obtain that

$$
(t, x) \rightarrow \int_{\mathbb{R}^{d}} p_{y}(t, x-y) f(y) d y
$$

is continuous on $(0, \tau+1] \times \mathbb{R}^{d}$. It follows that

$$
(\xi, t, x) \rightarrow \int_{\mathbb{R}^{d}} p_{y}(t+\xi, x-y) f(y) d y
$$


is continuous on $[0,1] \times(0, \tau] \times \mathbb{R}^{d}$. Using Lemma 3.4, Corollary 3.3, Proposition 3.12 and the dominated convergence theorem we obtain that for any $s \in(0, \tau)$

$$
(\xi, t, x) \rightarrow \int_{\mathbb{R}^{d}} p_{z}(t+\xi-s, x-z) Q_{s} f(z) d z
$$

is continuous on $[0,1] \times(s, \tau] \times \mathbb{R}^{d}$. Using this, Corollary 3.3, Proposition 3.12 and the dominated convergence theorem we obtain that

$$
(\xi, t, x) \rightarrow \Phi_{t}^{(\xi)} f(x)=\int_{0}^{\tau} 1_{(0, t)}(s) \int_{\mathbb{R}^{d}} p_{z}(t+\xi-s, x-z) Q_{s} f(z) d z d s
$$

is continuous on $[0,1] \times(0, \tau] \times \mathbb{R}^{d}$. Hence $(\xi, t, x) \rightarrow U_{t}^{(\xi)} f(x)$ is continuous on $[0,1] \times$ $(0, \tau] \times \mathbb{R}^{d}$. Using (71) we obtain that

$$
(\xi, t, x) \rightarrow U_{t}^{(\xi)} f(x) \text { is continuous on }[0,1] \times[0, \tau] \times \mathbb{R}^{d} .
$$

This and (iii) imply (iv).

By the same arguments as in the proof of Lemma 3.19 (iv) we obtain the following result.

Lemma 3.20 For any $f \in \mathcal{B}_{b}\left(\mathbb{R}^{d}\right)$ the function $(t, x) \rightarrow U_{t} f(x)$ is continuous on $(0, \infty) \times$ $\mathbb{R}^{d}$. For any $\xi \in(0,1], f \in \mathcal{B}_{b}\left(\mathbb{R}^{d}\right)$ the function $(t, x) \rightarrow U_{t}^{(\xi)} f(x)$ is continuous on $[0, \infty) \times \mathbb{R}^{d}$.

Heuristically, now our aim is to show that if $\xi$ is small then $\frac{\partial}{\partial t}\left(U_{t}^{(\xi)} f\right)(x)-\mathcal{L}\left(U_{t}^{(\xi)} f\right)(x)$ is small. For any $t>0, \xi \in(0,1], x \in \mathbb{R}^{d}$ we put

$$
\Lambda_{t}^{(\xi)} f(x)=\frac{\partial}{\partial t}\left(U_{t}^{(\xi)} f\right)(x)-\mathcal{L}\left(U_{t}^{(\xi)} f\right)(x) .
$$

Lemma 3.21 $\mathcal{L}\left(U_{t}^{(\xi)} f\right)(x)$ is well defined for every $f \in C_{0}\left(\mathbb{R}^{d}\right), t \in(0, \tau], \xi \in(0,1]$ and $x \in \mathbb{R}^{d}$ and we have

$$
\Lambda_{t}^{(\xi)} f(x)=\int_{\mathbb{R}^{d}} p_{z}(\xi, x-z) Q_{t} f(z) d z-Q_{t+\xi} f(x)+\int_{t}^{t+\xi} \int_{\mathbb{R}^{d}} q_{0}(t-s+\xi, x, z) Q_{s} f(z) d z d s .
$$

Moreover, we have

$$
\begin{gathered}
\left|\mathcal{L}_{\zeta}\left(U_{t}^{(\xi)} f\right)(x)\right| \leq c(\xi)\|f\|_{\infty}, \quad \zeta>0, \\
\left|\mathcal{L}\left(U_{t}^{(\xi)} f\right)(x)\right| \leq c(\xi)\|f\|_{\infty},
\end{gathered}
$$

for each $f \in C_{0}\left(\mathbb{R}^{d}\right), x \in \mathbb{R}^{d}, t \in(0, \tau], \xi \in(0,1]$, where $c(\xi)$ is a constant depending on $\xi, \tau, \alpha, d, \eta_{1}, \eta_{2}, \eta_{3}, \varepsilon$.

Proof Let $f \in C_{0}\left(\mathbb{R}^{d}\right), t \in(0, \tau], \xi \in(0,1], x \in \mathbb{R}^{d}$ and $\zeta>0$. We have

$$
\begin{aligned}
\mathcal{L}_{\zeta}\left(U_{t}^{(\xi)} f\right)(x)= & \int_{\mathbb{R}^{d}} \mathcal{L}_{\zeta}^{x} p_{z}(t+\xi, \cdot)(x-z) f(z) d z \\
& +\int_{0}^{t} \int_{\mathbb{R}^{d}} \mathcal{L}_{\zeta}^{x} p_{z}(t-s+\xi, \cdot)(x-z) Q_{s} f(z) d z d s
\end{aligned}
$$


Using this, Lemma 3.14 and Proposition 3.12 we obtain (74). By (76), the dominated convergence theorem, Lemma 3.14 and Proposition 3.12, one gets

$$
\begin{aligned}
\mathcal{L}\left(U_{t}^{(\xi)} f\right)(x)= & \lim _{\zeta \rightarrow 0^{+}} \mathcal{L}_{\zeta}^{x}\left(U_{t}^{(\xi)} f\right)(x) \\
= & \int_{\mathbb{R}^{d}} \mathcal{L}^{x} p_{z}(t+\xi, \cdot)(x-z) f(z) d z \\
& +\int_{0}^{t} \int_{\mathbb{R}^{d}} \mathcal{L}^{x} p_{z}(t-s+\xi, \cdot)(x-z) Q_{s} f(z) d z d s .
\end{aligned}
$$

Using this and again Lemma 3.14 and Proposition 3.12 we obtain (75).

Note that for $s \in[0, t), z \in \mathbb{R}^{d}$ we have

$$
\frac{\partial p_{z}(t-s+\xi, x-z)}{\partial t}-\mathcal{L}^{x} p_{z}(t-s+\xi, \cdot)(x-z)=-q_{0}(t-s+\xi, x, z) .
$$

Using this, (70) and (77) we get

$$
\begin{aligned}
\Lambda_{t}^{(\xi)} f(x)= & \int_{\mathbb{R}^{d}} p_{z}(\xi, x-z) Q_{t} f(z) d z \\
& -\int_{\mathbb{R}^{d}} q_{0}(t+\xi, x, z) f(z) d z \\
& -\int_{0}^{t} \int_{\mathbb{R}^{d}} q_{0}(t-s+\xi, x, z) Q_{s} f(z) d z d s .
\end{aligned}
$$

For $\xi \in(0,1], t \in(0, \tau], x \in \mathbb{R}^{d}$, by the definition of $q(t, x, y)$, we obtain

$$
\int_{\mathbb{R}^{d}} q_{0}(t+\xi, x, z) f(z) d z=Q_{t+\xi} f(x)-\int_{0}^{t+\xi} \int_{\mathbb{R}^{d}} q_{0}(t-s+\xi, x, z) Q_{s} f(z) d z d s .
$$

Using this and (78) we obtain (73).

The next lemma is similar to [19, Lemma 4.2].

Lemma 3.22 (i) For any $f \in C_{0}\left(\mathbb{R}^{d}\right)$ we have

$$
\Lambda_{t}^{(\xi)} f(x) \rightarrow 0, \quad \text { as } \xi \rightarrow 0^{+},
$$

uniformly in $(t, x) \in\left[\tau_{1}, \tau_{2}\right] \times \mathbb{R}^{d}$ for every $0<\tau_{1}<\tau_{2} \leq \tau$.

(ii) For any $f \in C_{0}\left(\mathbb{R}^{d}\right)$ we have

$$
\int_{0}^{t} \Lambda_{s}^{(\xi)} f(x) d s \rightarrow 0, \quad \text { as } \quad \xi \rightarrow 0^{+}
$$

uniformly in $(t, x) \in(0, \tau] \times \mathbb{R}^{d}$.

Proof Let $f \in C_{0}\left(\mathbb{R}^{d}\right)$ and $0<\tau_{1}<\tau_{2} \leq \tau$. For any $t>0, x \in \mathbb{R}^{d}, \xi \in(0,1]$ we put

$$
\begin{aligned}
\Lambda_{t}^{(\xi, 1)} f(x) & =\int_{\mathbb{R}^{d}} p_{z}(\xi, x-z) Q_{t} f(z) d z-Q_{t+\xi} f(x) . \\
\Lambda_{t}^{(\xi, 2)} f(x) & =\int_{t}^{t+\xi} \int_{\mathbb{R}^{d}} q_{0}(t-s+\xi, x, z) Q_{s} f(z) d z d s .
\end{aligned}
$$

By Lemma 3.17, we get

$$
\sup _{t \in\left[\tau_{1}, \tau_{2}\right], x \in \mathbb{R}^{d}}\left|Q_{t+\xi} f(x)-Q_{t} f(x)\right| \rightarrow 0 \quad \text { as } \quad \xi \rightarrow 0^{+} .
$$


By Lemmas 3.15 and 3.17, we obtain

$$
\sup _{t \in\left[\tau_{1}, \tau_{2}\right], x \in \mathbb{R}^{d}}\left|\int_{\mathbb{R}^{d}} p_{z}(\xi, x-z) Q_{t} f(z) d z-Q_{t} f(x)\right| \rightarrow 0 \quad \text { as } \quad \xi \rightarrow 0^{+} .
$$

This gives (i) for $\Lambda_{t}^{(\xi, 1)} f(x)$ instead of $\Lambda_{t}^{(\xi)} f(x)$.

By Proposition 3.12, for any $t \in(0, \tau], x \in \mathbb{R}^{d}, \xi \in(0,1]$ we get

$$
\left|\Lambda_{t}^{(\xi, 1)} f(x)\right| \leq c\|f\|_{\infty} t^{-1 / 2} \text {. }
$$

This allows to use the dominated convergence theorem in the integral (79) with $\Lambda_{t}^{(\xi)} f(x)$ replaced by $\Lambda_{t}^{(\xi, 1)} f(x)$. So (ii) for $\Lambda_{t}^{(\xi, 1)} f(x)$ follows from (i) for $\Lambda_{t}^{(\xi, 1)} f(x)$.

For any $t \in(0, \tau], x \in \mathbb{R}^{d}, \xi \in(0,1]$, by Propositions 3.9 and 3.12, we get

$$
\left|\Lambda_{t}^{(\xi, 2)} f(x)\right| \leq c\|f\|_{\infty} \int_{t}^{t+\xi}((t-s+\xi) s)^{-1 / 2} d s .
$$

This implies (i) and (ii) for $\Lambda_{t}^{(\xi, 2)} f(x)$.

Lemma 3.23 There exist $\varepsilon_{1} \in(0,1]$ and $t_{1} \in(0,1]$ such that for any $t \in\left(0, t_{1}\right], x, y \in \mathbb{R}^{d}$, $|x-y| \leq \varepsilon_{1} t^{1 / \alpha}$ we have

$$
u(t, x, y) \geq c_{1} t^{-d / \alpha}
$$

$\varepsilon_{1}, t_{1}$ depend on $\alpha, d, \eta_{1}, \eta_{2}, \eta_{3}, \varepsilon$.

Proof By the weak lower scaling property of the symbol $\Phi_{\delta}^{(1)}$ (see proof of Lemma 2.1) and by [3, formula (23)], we get that $g_{t}(0) \geq c t^{-1 / \alpha}$. Using this and Lemma 2.1 there exist $\varepsilon_{2}>0, t_{2}>0$ such that for $|y| \leq \varepsilon_{2} t^{1 / \alpha}, t \leq t_{2}$ we have $g_{t}(y) \geq c t^{-1 / \alpha}$. It follows that there exist $\varepsilon_{3}>0, t_{3}>0$ such that for $x, y \in \mathbb{R}^{d},|x-y| \leq \varepsilon_{3} t^{1 / \alpha}, t \leq t_{3}$ we have $p_{y}(t, x-y) \geq c t^{-d / \alpha}$. By Lemma 3.12 and Proposition 3.10, for $t \in(0, \tau], x, y \in \mathbb{R}^{d}$ and $a \in[1 / 2,1)$, we get

$$
\begin{aligned}
\left|\varphi_{y}(t, x)\right|= & \mid \int_{0}^{t a} \int_{\mathbb{R}^{d}} p_{z}(t-s, x-z) q(s, z, y) d z d s \\
& +\int_{t a}^{t} \int_{\mathbb{R}^{d}} p_{z}(t-s, x-z) q(s, z, y) d z d s \mid \\
\leq & c(t-t a)^{-d / \alpha} \int_{0}^{t a} s^{-1 / 2} d s+c t^{-d / \alpha-1} t(1-a) \\
\leq & c t^{-d / \alpha}\left(t^{1 / 2}(1-a)^{-d / \alpha}+1-a\right) .
\end{aligned}
$$

By an appropriate choice of $a$, there exists $\varepsilon_{1}$ and $t_{1}>0$ such that for any $x, y \in \mathbb{R}^{d}$, $|x-y| \leq \varepsilon_{1} t^{1 / \alpha}, t \leq t_{1}$ we have

$u(t, x, y)=p_{y}(t, x-y)-\varphi_{y}(t, x) \geq c t^{-d / \alpha}-c_{2} t^{-d / \alpha}\left(t^{1 / 2}(1-a)^{-d / \alpha}+1-a\right) \geq c_{1} t^{-d / \alpha}$. 


\section{Construction and Properties of the Semigroup of $X_{t}$}

Let us introduce the following notation

$$
\begin{gathered}
v(x)=\frac{\mathcal{A}_{\alpha}}{|x|^{1+\alpha}}-\mu(x), \quad x \in \mathbb{R}, \\
\lambda=d \int_{\mathbb{R}} v(x) d x<\infty .
\end{gathered}
$$

Note that by (7), for any $x \in \mathbb{R}^{d}$ and $f \in \mathcal{B}_{b}\left(\mathbb{R}^{d}\right)$, we have

$$
\mathcal{R} f(x)=\sum_{i=1}^{d} \int_{\mathbb{R}}\left[f\left(x+a_{i}(x) w\right)-f(x)\right] v(w) d w .
$$

We denote, for any $x \in \mathbb{R}^{d}$ and $f \in \mathcal{B}_{b}\left(\mathbb{R}^{d}\right)$,

$$
\mathcal{N} f(x)=\sum_{i=1}^{d} \int_{\mathbb{R}}\left[f\left(x+a_{i}(x) w\right)\right] v(w) d w .
$$

It is clear that

$$
\|\mathcal{N} f\|_{\infty} \leq \lambda\|f\|_{\infty} .
$$

For any $t \geq 0, x \in \mathbb{R}^{d}$ and $n \in \mathbb{N}, n \geq 1, f \in \mathcal{B}_{b}\left(\mathbb{R}^{d}\right)$ we define

$$
\begin{aligned}
& \Psi_{0, t} f(x)=U_{t} f(x), \\
& \Psi_{n, t} f(x)=\int_{0}^{t} U_{t-s}\left(\mathcal{N}\left(\Psi_{n-1, s} f\right)\right)(x) d s, \quad n \geq 1 .
\end{aligned}
$$

For any $t \geq 0, \xi \in[0,1], x \in \mathbb{R}^{d}$ and $n \in \mathbb{N}, f \in \mathcal{B}_{b}\left(\mathbb{R}^{d}\right)$ we define

$$
\begin{aligned}
& \Psi_{0, t}^{(\xi)} f(x)=U_{t}^{(\xi)} f(x), \\
& \Psi_{n, t}^{(\xi)} f(x)=\int_{0}^{t} U_{t-s}^{(\xi)}\left(\mathcal{N}\left(\Psi_{n-1, s}^{(\xi)} f\right)\right)(x) d s, \quad n \geq 1 .
\end{aligned}
$$

We observe that

$$
\Psi_{n, t}=\Psi_{n, t}^{(0)} .
$$

Finally, we remark that all these functions have nothing in common with the functions $\Psi$ or $\Psi_{x}$ used in Section 3 .

Lemma 4.1 $\Psi_{n, t} f(x)$ and $\Psi_{n, t}^{(\xi)} f(x)$ are well defined for any $t>0, f \in \mathcal{B}_{b}\left(\mathbb{R}^{d}\right), x \in \mathbb{R}^{d}$, $n \in \mathbb{N}$ and $\xi \in[0,1]$. For any $f \in \mathcal{B}_{b}\left(\mathbb{R}^{d}\right), x \in \mathbb{R}^{d}, n \in \mathbb{N}$ we have

$$
\begin{aligned}
& \left|\Psi_{n, t} f(x)\right| \leq \frac{c_{1}^{n+1} t^{n}}{n !}\|f\|_{\infty}, \quad t \in(0, \tau], \\
& \left|\Psi_{n, t}^{(\xi)} f(x)\right| \leq \frac{c_{1}^{n+1} t^{n}}{n !}\|f\|_{\infty}, \quad \xi \in(0,1], \quad t \in[0, \tau] .
\end{aligned}
$$

Proof We will only show the result for $\Psi_{n, t} f(x)$ using the induction. The proof for $\Psi_{n, t}^{(\xi)} f(x)$ is almost the same. 
Let $c$ be the constant from (59) and put $c_{1}=(\lambda \vee 1) c$. For $n=0$ (87) follows from (59). Assume that (87) holds for $n \geq 0$, we will show it for $n+1$. Indeed, applying (59) and (82), we get

$$
\left|\Psi_{n+1, t} f(x)\right| \leq \int_{0}^{t} \int_{\mathbb{R}^{d}}|u(t-s, x, z)| d z \frac{\lambda c_{1}^{n+1} s^{n}}{n !} d s \leq \frac{c_{1}^{n+2} t^{n+1}}{(n+1) !} .
$$

For any $x \in \mathbb{R}^{d}$ we define

$$
\begin{aligned}
T_{t} f(x) & =e^{-\lambda t} \sum_{n=0}^{\infty} \Psi_{n, t} f(x), \quad t>0, \\
T_{0} f(x) & =f(x), \\
T_{t}^{(\xi)} f(x) & =e^{-\lambda t} \sum_{n=0}^{\infty} \Psi_{n, t}^{(\xi)} f(x), \quad t \geq 0, \quad \xi \in[0,1] .
\end{aligned}
$$

Our ultimate aim will be to show that for any $t>0$ we have $T_{t}=P_{t}$, where $P_{t}$ is given by (5).

By Lemma 4.1, we obtain

Corollary 4.2 $T_{t} f(x)$ and $T_{t}^{(\xi)} f(x)$ are well defined for any $t \geq 0, f \in \mathcal{B}_{b}\left(\mathbb{R}^{d}\right), x \in \mathbb{R}^{d}$, $\xi \in[0,1]$ and for $t \in[0, \tau]$ we have $\max \left\{\left|T_{t} f(x)\right|,\left|T_{t}^{(\xi)} f(x)\right|\right\} \leq c\|f\|_{\infty}$.

Next, we obtain the following regularity results concerning operators $T_{t}$.

Theorem 4.3 For any $\gamma \in(0, \alpha / d), t \in(0, \tau], x \in \mathbb{R}^{d}$ and $f \in L^{1}\left(\mathbb{R}^{d}\right) \cap L^{\infty}\left(\mathbb{R}^{d}\right)$ we have

$$
\left|T_{t} f(x)\right| \leq c t^{-\gamma d / \alpha}\|f\|_{\infty}^{1-\gamma}\|f\|_{1}^{\gamma} .
$$

Proof For any $t \in(0, \tau], x \in \mathbb{R}^{d}$ by Corollary 3.13 we get $\left|U_{t} f(x)\right| \leq c\|f\|_{\infty}$, $\left|U_{t} f(x)\right| \leq c t^{-d / \alpha}\|f\|_{1}$. Fix $\gamma \in(0, \alpha / d)$. It follows that for any $t \in(0, \tau], x \in \mathbb{R}^{d}$ we have $\left|U_{t} f(x)\right| \leq c t^{-\gamma d / \alpha}\|f\|_{\infty}^{1-\gamma}\|f\|_{1}^{\gamma}$. Hence $\left|\Psi_{1, t} f(x)\right| \leq c\|f\|_{\infty}^{1-\gamma}\|f\|_{1}^{\gamma}$. Using the same arguments as in Lemma 4.1 for any $t \in(0, \tau], x \in \mathbb{R}^{d}, n \in \mathbb{N}, n \geq 1$ one gets $\left|\Psi_{n, t} f(x)\right| \leq c^{n} t^{n-1}\|f\|_{\infty}^{1-\gamma}\|f\|_{1}^{\gamma} /(n-1)$ !, which implies the assertion of the theorem.

Theorem 4.4 Choose $\gamma \in(0, \alpha)$. For any $t \in(0, \tau], x, x^{\prime} \in \mathbb{R}^{d}, f \in \mathcal{B}_{b}\left(\mathbb{R}^{d}\right)$ we have

$$
\left|T_{t} f(x)-T_{t} f\left(x^{\prime}\right)\right| \leq c t^{-\gamma / \alpha}\left|x-x^{\prime}\right|^{\gamma}\|f\|_{\infty} .
$$

Proof We have

$$
T_{t} f(x)=e^{-\lambda t} U_{t} f(x)+e^{-\lambda t} \sum_{n=1}^{\infty} \Psi_{n, t} f(x) .
$$

By Lemma 3.18, it remains to show

$$
\left|\sum_{n=1}^{\infty} \Psi_{n, t} f(x)-\sum_{n=1}^{\infty} \Psi_{n, t} f\left(x^{\prime}\right)\right| \leq c t^{-\gamma / \alpha}\left|x-x^{\prime}\right|^{\gamma}\|f\|_{\infty} .
$$


Let $n \in \mathbb{N}, n \geq 1$. By (84) we get

$$
\Psi_{n, t} f(x)-\Psi_{n, t} f\left(x^{\prime}\right)=\int_{0}^{t}\left(U_{t-s}\left(\mathcal{N}\left(\Psi_{n-1, s} f\right)\right)(x)-U_{t-s}\left(\mathcal{N}\left(\Psi_{n-1, s} f\right)\right)\left(x^{\prime}\right)\right) d s .
$$

By Lemma 4.1, we have

$$
\left|\Psi_{n, t} f(x)\right| \leq \frac{c_{1}^{n+1} t^{n}}{n !}\|f\|_{\infty} .
$$

Hence for $s \in(0, \tau]$, by (82), we arrive at

$$
\left.\mid \mathcal{N}\left(\Psi_{n-1, s} f\right)\right)(x) \mid \leq \frac{\lambda c_{1}^{n} s^{n-1}}{(n-1) !}\|f\|_{\infty} .
$$

Using this and Lemma 3.18 we get

$$
\left|U_{t-s}\left(\mathcal{N}\left(\Psi_{n-1, s} f\right)\right)(x)-U_{t-s}\left(\mathcal{N}\left(\Psi_{n-1, s} f\right)\right)\left(x^{\prime}\right)\right| \leq c(t-s)^{-\gamma / \alpha}\left|x-x^{\prime}\right|^{\gamma} \frac{c_{1}^{n} s^{n-1}}{(n-1) !}\|f\|_{\infty} .
$$

Combining this with (90) we obtain

$$
\left|\Psi_{n, t} f(x)-\Psi_{n, t} f\left(x^{\prime}\right)\right| \leq c \frac{c_{1}^{n}}{(n-1) !}\|f\|_{\infty}\left|x-x^{\prime}\right|^{\gamma} t^{n-\gamma / \alpha} .
$$

This implies (89), which finishes the proof.

Clearly, we have, by applying (59) and (82), the following lemma.

Lemma 4.5 There exists $a \geq 1$ such that for any $f \in \mathcal{B}_{b}\left(\mathbb{R}^{d}\right), x \in \mathbb{R}^{d}, \xi \in[0,1]$, $t \in(0, \tau]$ we have

$$
\left|\int_{0}^{t} U_{t-s}^{(\xi)}(\mathcal{N}(f))(x) d s\right| \leq a\|f\|_{\infty},
$$

where a depends on $\tau, \alpha, d, \eta_{1}, \eta_{2}, \eta_{3}$.

Lemma 4.6 Assume that $f \in \mathcal{B}_{b}\left(\mathbb{R}^{d}\right)$. Put $\beta=1 /(4(d / \alpha+1))$. For any $t \in(0, \tau]$, $x \in \mathbb{R}^{d}$, we have

$$
\left|Q_{t} f(x)\right| \leq \frac{c\|f\|_{\infty}}{t^{3 / 4}(\operatorname{dist}(x, \operatorname{supp}(f))+1)^{\beta}} .
$$

Proof Let $t \in(0, \tau]$ be arbitrary. By Proposition 3.12, we get for $x \in \mathbb{R}^{d}$,

$$
\begin{aligned}
\left|Q_{t} f(x)\right| & \leq c t^{-1 / 2}\|f\|_{\infty}, \\
\left|Q_{t} f(x)\right| & \leq \frac{c\|f\|_{\infty}}{t^{d / \alpha+1}(\operatorname{dist}(x, \operatorname{supp}(f))+1)} .
\end{aligned}
$$

It follows that

$$
\begin{aligned}
\left|Q_{t} f(x)\right|^{1-\beta} & \leq c t^{(-1 / 2)(1-\beta)}\|f\|_{\infty}^{1-\beta} \leq c t^{-1 / 2}\|f\|_{\infty}^{1-\beta}, \\
\left|Q_{t} f(x)\right|^{\beta} & \leq \frac{c\|f\|_{\infty}^{\beta}}{t^{1 / 4}(\operatorname{dist}(x, \operatorname{supp}(f))+1)^{\beta}} .
\end{aligned}
$$

This implies the assertion of the lemma. 
Lemma 4.7 Assume that $f \in \mathcal{B}_{b}\left(\mathbb{R}^{d}\right)$. For any $\varepsilon_{1}>0$ there exists $r \geq 1$ (depending on $\left.\varepsilon_{1}, \tau, \alpha, d, \eta_{1}, \eta_{2}, \eta_{3}\right)$ such that for any $\xi \in[0,1], t \in[0, \tau], x \in \mathbb{R}^{d}$, if $\operatorname{dist}(x, \operatorname{supp}(f)) \geq$ $r$, then $\left|U_{t}^{(\xi)} f(x)\right| \leq \varepsilon_{1}\|f\|_{\infty}$.

Proof Let $\xi \in[0,1], t \in[0, \tau]$ be arbitrary. Assume that $\operatorname{dist}(x, \operatorname{supp}(f)) \geq 1$ and $t+\xi>$ 0 . By Lemmas 3.10, 4.6 we get

$$
\begin{aligned}
\left|\Phi_{t}^{(\xi)} f(x)\right| & =\left|\int_{0}^{t} \int_{\mathbb{R}^{d}} p_{z}(t-s+\xi, x-z) Q_{s} f(z) d z d s\right| \\
& \leq \frac{c\|f\|_{\infty}}{(\operatorname{dist}(x, \operatorname{supp}(f))+1)^{\beta}} \int_{0}^{t} \frac{1}{s^{3 / 4}} d s .
\end{aligned}
$$

By Corollary 3.3 we get

$$
\int_{\mathbb{R}^{d}} p_{z}(t+\xi, x-z) f(z) d z \leq c\|f\|_{\infty} e^{-c_{1} \operatorname{dist}(x, \operatorname{supp}(f))} .
$$

This gives the assertion of the lemma.

The proof of the next lemma is standard and it is omitted.

Lemma 4.8 Assume that $f \in \mathcal{B}_{b}\left(\mathbb{R}^{d}\right)$. For any $\varepsilon_{1}>0$ there exists $r \geq 1$ (depending on $\left.\varepsilon_{1}, \tau, \alpha, d, \eta_{1}, \eta_{2}, \eta_{3}\right)$ such that, for any $x \in \mathbb{R}^{d}$, if $\operatorname{dist}(x, \operatorname{supp}(f)) \geq r$, then $|\mathcal{N} f(x)| \leq$ $\varepsilon_{1}\|f\|_{\infty}$.

Lemma 4.9 Assume that $f \in \mathcal{B}_{b}\left(\mathbb{R}^{d}\right)$. For any $\varepsilon_{1}>0$ there exists $r \geq 1$ (depending on $\left.\varepsilon_{1}, \tau, \alpha, d, \eta_{1}, \eta_{2}, \eta_{3}\right)$ such that for any $\xi \in[0,1], t \in[0, \tau], x \in \mathbb{R}^{d}$, if $\operatorname{dist}(x, \operatorname{supp}(f)) \geq$ $r$, then $\left|\int_{0}^{t} U_{t-s}^{(\xi)}(\mathcal{N}(f))(x) d s\right| \leq \varepsilon_{1}\|f\|_{\infty}$.

Proof Let $\xi \in[0,1], t \in(0, \tau]$ be arbitrary. Choose $\varepsilon_{1}>0$. There exists $a>0$ such that for any $g \in \mathcal{B}_{b}\left(\mathbb{R}^{d}\right)$ we have $\left\|U_{t}^{(\xi)} g\right\|_{\infty} \leq a\|g\|_{\infty}$, where $a$ depends on $\tau, \alpha, d, \eta_{1}, \eta_{2}, \eta_{3}$. By Lemma 4.8, there exists $r_{1} \geq 1$ such that, if $\operatorname{dist}(x, \operatorname{supp}(f)) \geq r_{1}$, then

$$
|\mathcal{N} f(x)| \leq \frac{\varepsilon_{1}\|f\|_{\infty}}{2 a \tau},
$$

where $r_{1}$ depends on $\varepsilon_{1}, \tau, \alpha, d, \eta_{1}, \eta_{2}, \eta_{3}$. Put $A=\left\{x \in \mathbb{R}^{d}: \operatorname{dist}(x, \operatorname{supp}(f)) \geq r_{1}\right\}$. We have, applying also (82),

$$
|\mathcal{N} f(x)|=\left|\mathcal{N} f(x) \mathbf{1}_{A^{c}}(x)+\mathcal{N} f(x) \mathbf{1}_{A}(x)\right| \leq \lambda\|f\|_{\infty} \mathbf{1}_{A^{c}}(x)+\frac{\varepsilon_{1}\|f\|_{\infty}}{2 a \tau} \mathbf{1}_{A}(x) .
$$

Put $\tilde{f}(x)=\lambda\|f\|_{\infty} \mathbf{1}_{A^{c}}(x), \tilde{\tilde{f}}(x)=\frac{\varepsilon_{1}\|f\|_{\infty}}{2 a \tau} \mathbf{1}_{A}(x)$.

We have

$$
\left|\int_{0}^{t} U_{t-s}^{(\xi)}(\mathcal{N}(f))(x) d s\right| \leq \int_{0}^{t} U_{t-s}^{(\xi)}(\tilde{f})(x) d s+\int_{0}^{t} U_{t-s}^{(\xi)}(\tilde{\tilde{f}})(x) d s .
$$

For any $x \in \mathbb{R}^{d}$ we get

$$
\int_{0}^{t} U_{t-s}^{(\xi)}(\tilde{\tilde{f}})(x) d s \leq \frac{\varepsilon_{1}\|f\|_{\infty}}{2}
$$


By Lemma 4.7, there exists $r_{2} \geq r_{1}$ such that, if $\operatorname{dist}(x, \operatorname{supp}(f)) \geq r_{2}$ and $s \in(0, t)$, then

$$
\left|U_{t-s}^{(\xi)}(\tilde{f})(x)\right| \leq \frac{\varepsilon_{1}\|f\|_{\infty}}{2 \tau},
$$

where $r_{2}$ depends on $\varepsilon_{1}, r_{1}, \tau, \alpha, d, \eta_{1}, \eta_{2}, \eta_{3}$. It follows that, if $\operatorname{dist}(x, \operatorname{supp}(f)) \geq r_{2}$, then

$$
\int_{0}^{t} U_{t-s}^{(\xi)}(\tilde{f})(x) d s \leq \frac{\varepsilon_{1}\|f\|_{\infty}}{2} .
$$

Finally, (91-93) imply the assertion of the lemma.

Lemma 4.10 Assume that $f \in \mathcal{B}_{b}\left(\mathbb{R}^{d}\right)$. For any $\varepsilon_{1}>0$ there exists $r \geq 1$ (depending on $\left.\varepsilon_{1}, \tau, \alpha, d, \eta_{1}, \eta_{2}, \eta_{3}\right)$, such that for any $\xi \in[0,1], t \in[0, \tau], x \in \mathbb{R}^{d}$, if $\operatorname{dist}(x, \operatorname{supp}(f)) \geq$ $r$, then $\left|T_{t}^{(\xi)} f(x)\right| \leq \sum_{n=0}^{\infty}\left|\Psi_{n, t}^{(\xi)} f(x)\right| \leq \varepsilon_{1}\|f\|_{\infty}$.

Proof Fix $f \in \mathcal{B}_{b}\left(\mathbb{R}^{d}\right)$. Put

$$
M=\sup _{n \in \mathbb{N}} \frac{c_{1}^{n+1} \tau^{n}}{n !},
$$

where $c_{1}$ is a constant from Lemma 4.1. Let $\xi \in[0,1], t \in[0, \tau]$ be arbitrary such that $t+\xi>0$. Choose $\varepsilon_{2}>0$. By Lemma 4.1, there exists $n_{0}$ such that for any $x \in \mathbb{R}^{d}$ we have

$$
\sum_{n=n_{0}}^{\infty}\left|\Psi_{n, t}^{(\xi)} f(x)\right| \leq \varepsilon_{2}\|f\|_{\infty} .
$$

Put $r_{-1}=1$. Now we will show that for any $n \in \mathbb{N}$ there exists $r_{n} \geq r_{n-1}$ such that, if $\operatorname{dist}(x, \operatorname{supp}(f)) \geq r_{n}$, then

$$
\left|\Psi_{n, t}^{(\xi)} f(x)\right| \leq 2^{n} a^{n} \varepsilon_{2}\|f\|_{\infty},
$$

where $a \geq 1$ is a constant from Lemma 4.5 and $r_{n}$ depends on $r_{n-1}, \varepsilon_{2}, \tau, \alpha, d, \eta_{1}, \eta_{2}, \eta_{3}$.

By Lemma 4.7, there exists $r_{0} \geq r_{-1}$ such that, if $\operatorname{dist}(x, \operatorname{supp}(f)) \geq r_{0}$, then

$$
\left|\Psi_{0, t}^{(\xi)} f(x)\right|=\left|U_{t}^{(\xi)} f(x)\right| \leq \varepsilon_{2}\|f\|_{\infty}
$$

where $r_{0}$ depends on $\varepsilon_{2}, \tau, \alpha, d, \eta_{1}, \eta_{2}, \eta_{3}$.

Assume that (95) holds for $n \in \mathbb{N}$. We will show it for $n+1$. Put $A_{n}=\left\{x \in \mathbb{R}^{d}\right.$ : $\left.\operatorname{dist}(x, \operatorname{supp}(f)) \geq r_{n}\right\}$. We have

$$
\begin{aligned}
\left|\Psi_{n, t}^{(\xi)} f(x)\right| & =\left|\Psi_{n, t}^{(\xi)} f(x) \mathbf{1}_{A_{n}^{c}}(x)+\Psi_{n, t}^{(\xi)} f(x) \mathbf{1}_{A_{n}}(x)\right| \\
& \leq M\|f\|_{\infty} \mathbf{1}_{A_{n}^{c}}(x)+2^{n} a^{n} \varepsilon_{2}\|f\|_{\infty} \mathbf{1}_{A_{n}}(x) .
\end{aligned}
$$

Put $f_{n}(x)=M\|f\|_{\infty} \mathbf{1}_{A_{n}^{c}}(x), \tilde{f}_{n}(x)=2^{n} a^{n} \varepsilon_{2}\|f\|_{\infty} \mathbf{1}_{A_{n}}(x)$.

We have

Hence,

$$
\Psi_{n+1, t}^{(\xi)} f(x)=\int_{0}^{t} U_{t-s}^{(\xi)}\left(\mathcal{N}\left(\Psi_{n, s}^{(\xi)} f\right)\right)(x) d s .
$$

$$
\left|\Psi_{n+1, t}^{(\xi)} f(x)\right| \leq \int_{0}^{t} U_{t-s}^{(\xi)}\left(\mathcal{N}\left(f_{n}\right)\right)(x) d s+\int_{0}^{t} U_{t-s}^{(\xi)}\left(\mathcal{N}\left(\tilde{f}_{n}\right)\right)(x) d s .
$$

By Lemma 4.5 , for any $x \in \mathbb{R}^{d}$, we get

$$
\int_{0}^{t} U_{t-s}^{(\xi)}\left(\mathcal{N}\left(\tilde{f}_{n}\right)\right)(x) d s \leq a\left\|\tilde{f}_{n}\right\|_{\infty} \leq 2^{n} a^{n+1} \varepsilon_{2}\|f\|_{\infty} .
$$


By Lemma 4.9, there exists $r_{n+1} \geq r_{n}$ such that, if $\operatorname{dist}(x, \operatorname{supp}(f)) \geq r_{n+1}$, then

$$
\int_{0}^{t} U_{t-s}^{(\xi)}\left(\mathcal{N}\left(f_{n}\right)\right)(x) d s \leq \varepsilon_{2}\|f\|_{\infty},
$$

where $r_{n+1}$ depends on $\varepsilon_{2}, r_{n}, \tau, \alpha, d, \eta_{1}, \eta_{2}, \eta_{3}$.

By (97-99), we obtain (95) for $n+1$. By (95), we obtain that, if $\operatorname{dist}(x, \operatorname{supp}(f)) \geq r_{n_{0}}$, then

$$
\sum_{n=0}^{n_{0}}\left|\Psi_{n, t}^{(\xi)} f(x)\right| \leq \varepsilon_{2} \sum_{n=0}^{n_{0}} 2^{n} a^{n}\|f\|_{\infty} .
$$

Using this and (94) we get the assertion of the lemma.

By Lemma 4.10 and Theorem 4.3, one easily obtains the following result.

Corollary 4.11 Assume that $f \in \mathcal{B}_{b}\left(\mathbb{R}^{d}\right)$, for any $n \in \mathbb{N}, n \geq 1$ we have $f_{n} \in \mathcal{B}_{b}\left(\mathbb{R}^{d}\right)$, $\sup _{n \in \mathbb{N}, n \geq 1}\left\|f_{n}\right\|_{\infty}<\infty$ and $\lim _{n \rightarrow \infty} f_{n}(x)=f(x)$ for almost all $x \in \mathbb{R}^{d}$ with respect to the Lebesgue measure. Then, for any $t>0, x \in \mathbb{R}^{d}$, we have $\lim _{n \rightarrow \infty} T_{t} f_{n}(x)=T_{t} f(x)$.

Lemma 4.12 (i) For every $f \in C_{0}\left(\mathbb{R}^{d}\right)$ we have

$$
\lim _{t, \xi \rightarrow 0^{+}}\left\|T_{t}^{(\xi)} f-f\right\|_{\infty}=0 .
$$

(ii) For every $f \in C_{0}\left(\mathbb{R}^{d}\right)$ we have

$$
T_{t}^{(\xi)} f(x) \rightarrow 0, \quad \text { as } \quad|x| \rightarrow \infty,
$$

uniformly in $t \in[0, \tau], \xi \in[0,1]$.

(iii) For every $f \in C_{0}\left(\mathbb{R}^{d}\right)$ we have

$$
\left\|T_{t}^{(\xi)} f-T_{t} f\right\|_{\infty} \rightarrow 0, \quad \text { as } \xi \rightarrow 0^{+},
$$

uniformly in $t \in[0, \tau]$.

Proof (i) This follows from Lemma 3.19 (ii) and Lemma 4.1.

(ii) Note that $T_{0}^{(\xi)} f=U_{0}^{(\xi)}$ so (ii) for $t=0$ follows from Lemma 3.19. So we may assume that $t>0$. Let $t \in(0, \tau], \xi \in[0,1]$ be arbitrary. By Lemma 4.2, we have

$$
\left\|T_{t}^{(\xi)} f\right\|_{\infty} \leq c_{1}\|f\|_{\infty}
$$

Choose $\varepsilon_{1}>0$. Since $f \in C_{0}\left(\mathbb{R}^{2}\right)$, there exists $r_{1}>0$ such that if $|x| \geq r_{1}$ then $|f(x)| \leq \varepsilon_{1} /\left(2 c_{1}\right)$, where $c_{1}$ is a constant from (100). Put $f_{1}(x)=f(x) \mathbf{1}_{B\left(0, r_{1}\right)}(x)$, $f_{2}(x)=f(x) \mathbf{1}_{B^{c}\left(0, r_{1}\right)}(x)$. By Lemma 4.10, there exists $r_{2}>r_{1}$ such that, if $|x| \geq r_{2}$, then $\left|T_{t}^{(\xi)} f_{1}(x)\right| \leq \varepsilon_{1} / 2$. Hence for any $|x| \geq r_{2}$ we have $\left|T_{t}^{(\xi)} f(x)\right| \leq \mid T_{t}^{(\xi)} f_{1}(x)+$ $T_{t}^{(\xi)} f_{2}(x) \mid \leq \varepsilon_{1} / 2+\left(\varepsilon_{1} /\left(2 c_{1}\right)\right) c_{1}=\varepsilon_{1}$.

(iii) Let $\xi \in(0,1], n \in \mathbb{N}, n \geq 1, x \in \mathbb{R}^{d}, f \in C_{0}\left(\mathbb{R}^{d}\right)$. Note that for any $t \in(0, \tau]$ we have

$$
\begin{aligned}
\Psi_{n, t}^{(\xi)} f(x)= & \int_{0}^{t} \int_{\mathbb{R}^{d}} p_{y}(t-s+\xi, x-y) \mathcal{N}\left(\Psi_{n-1, s}^{(\xi)} f\right)(y) d y d s \\
& +\int_{0}^{t} \int_{0}^{t-s} \int_{\mathbb{R}^{d}} p_{z}(t-s-r+\xi, x-z) Q_{r}\left(\mathcal{N}\left(\Psi_{n-1, s}^{(\xi)} f\right)\right)(z) d z d r d s .
\end{aligned}
$$


By the same arguments as in the proof of Lemma 3.19 (iv), we obtain that $(\xi, t, x) \rightarrow$ $\Psi_{n, t}^{(\xi)} f(x)$ is continuous on $[0,1] \times[0, \tau] \times \mathbb{R}^{d}$ for any $n \in \mathbb{N}, n \geq 1$. Using this, (72) and Lemma 4.1 we obtain that $(\xi, t, x) \rightarrow T_{t}^{(\xi)} f(x)$ is continuous on $[0,1] \times[0, \tau] \times \mathbb{R}^{d}$. This and (ii) implies (iii).

By the same arguments as in the proof of Lemma 3.19 (iv), we obtain the following result.

Lemma 4.13 For any $f \in \mathcal{B}_{b}\left(\mathbb{R}^{d}\right), n \in \mathbb{N}$, the function $(t, x) \rightarrow \Psi_{n, t} f(x)$ is continuous on $(0, \infty) \times \mathbb{R}^{d}$. For any $\xi \in(0,1], f \in \mathcal{B}_{b}\left(\mathbb{R}^{d}\right), n \in \mathbb{N}$, the function $(t, x) \rightarrow \Psi_{n, t}^{(\xi)} f(x)$ is continuous on $[0, \infty) \times \mathbb{R}^{d}$.

Lemma $4.14 \frac{\partial}{\partial t}\left(\Psi_{n, t}^{(\xi)} f\right)(x), \mathcal{L}\left(\Psi_{n, t}^{(\xi)} f\right)(x)$ are well defined for any $t>0, \xi \in(0,1]$, $x \in \mathbb{R}^{d}, n \in \mathbb{N}, n \geq 1$ and $f \in C_{0}\left(\mathbb{R}^{d}\right)$ and we have

$$
\begin{aligned}
\frac{\partial}{\partial t}\left(\Psi_{n, t}^{(\xi)} f\right)(x)-\mathcal{L}\left(\Psi_{n, t}^{(\xi)} f\right)(x)= & \int_{\mathbb{R}^{d}} p_{z}(\xi, x-z) \mathcal{N}\left(\Psi_{n-1, t}^{(\xi)} f\right)(z) d z \\
& +\int_{0}^{t} \Lambda_{t-s}^{(\xi)}\left(\mathcal{N}\left(\Psi_{n-1, s}^{(\xi)} f\right)\right)(x) d s .
\end{aligned}
$$

Moreover, $\frac{\partial}{\partial t} \Psi_{n, t}^{(\xi)} f(x)$ is continuous as a function of $t$ for $t>0$.

Proof Let $\xi \in(0,1], n \in \mathbb{N}, n \geq 1, x \in \mathbb{R}^{d}, f \in C_{0}\left(\mathbb{R}^{d}\right)$. Note that for any $t \in(0, \tau]$, $s \in(0, t)$, we have

$$
\begin{aligned}
U_{t-s}^{(\xi)}\left(\mathcal{N}\left(\Psi_{n-1, s}^{(\xi)} f\right)\right)(x)= & \int_{\mathbb{R}^{d}} p_{y}(t-s+\xi, x-y) \mathcal{N}\left(\Psi_{n-1, s}^{(\xi)} f\right)(y) d y \\
& +\int_{0}^{t-s} \int_{\mathbb{R}^{d}} p_{z}(t-s-r+\xi, x-z) Q_{r}\left(\mathcal{N}\left(\Psi_{n-1, s}^{(\xi)} f\right)\right)(z) d z d r .
\end{aligned}
$$

By similar arguments as in the proof of Lemma 3.19 (i), we obtain that $\frac{\partial}{\partial t} U_{t-s}^{(\xi)}\left(\mathcal{N}\left(\Psi_{n-1, s}^{(\xi)} f\right)\right)(x)$ is well defined and continuous as a function of $t$ for $t \in(s, \tau]$. Note that for any $g \in C_{0}\left(\mathbb{R}^{d}\right)$, and $t \geq 0$ we have $U_{t}^{(\xi)} g \in C_{0}\left(\mathbb{R}^{d}\right), \mathcal{N} g \in C_{0}\left(\mathbb{R}^{d}\right)$. By (86), (69), Lemmas 3.20, 4.13, 3.19 (i) and standard arguments, we get

$$
\begin{aligned}
& \frac{\partial}{\partial t}\left(\Psi_{n, t}^{(\xi)} f\right)(x)=U_{0}^{(\xi)}\left(\mathcal{N}\left(\Psi_{n-1, t}^{(\xi)} f\right)\right)(x)+\int_{0}^{t} \frac{\partial}{\partial t} U_{t-s}^{(\xi)}\left(\mathcal{N}\left(\Psi_{n-1, s}^{(\xi)} f\right)\right)(x) d s \\
& =\int_{\mathbb{R}^{d}} p_{z}(\xi, x-z) \mathcal{N}\left(\Psi_{n-1, t}^{(\xi)} f\right)(z) d z+\int_{0}^{t} \Lambda_{t-s}^{(\xi)}\left(\mathcal{N}\left(\Psi_{n-1, s}^{(\xi)} f\right)\right)(x) d s \\
& \quad+\int_{0}^{t} \mathcal{L}^{x}\left(U_{t-s}^{(\xi)}\left(\mathcal{N}\left(\Psi_{n-1, s}^{(\xi)} f\right)\right)\right)(x) d s
\end{aligned}
$$

This implies that $\frac{\partial}{\partial t} \Psi_{n, t}^{(\xi)} f(x)$ is continuous as a function of $t$ for $t \in(0, \tau]$.

For any $\zeta>0$ we have

$$
\mathcal{L}_{\zeta}\left(\Psi_{n, t}^{(\xi)} f\right)(x)=\int_{0}^{t} \mathcal{L}_{\zeta}^{x} U_{t-s}^{(\xi)}\left(\mathcal{N}\left(\Psi_{n-1, s}^{(\xi)} f\right)\right)(x) d s .
$$


By the dominated convergence theorem and (74), we obtain

$$
\mathcal{L}\left(\Psi_{n, t}^{(\xi)} f\right)(x)=\lim _{\zeta \rightarrow 0^{+}} \mathcal{L}_{\zeta}\left(\Psi_{n, t}^{(\xi)} f\right)(x)=\int_{0}^{t} \mathcal{L}^{x} U_{t-s}^{(\xi)}\left(\mathcal{N}\left(\Psi_{n-1, s}^{(\xi)} f\right)\right)(x) d s .
$$

This and (102) gives the assertion of the lemma.

Lemma 4.15 For any $t>0, \xi \in(0,1], x \in \mathbb{R}^{d}, i, j \in\{1, \ldots, d\}, k \in \mathbb{N}$ and $f \in C_{0}\left(\mathbb{R}^{d}\right)$ we have $\Psi_{k, t}^{(\xi)} f(x) \in C^{2}\left(\mathbb{R}^{d}\right)$ (as a function of $x$ ) and

$$
\begin{aligned}
\frac{\partial}{\partial t}\left(\sum_{n=0}^{\infty} \Psi_{n, t}^{(\xi)} f\right)(x) & =\sum_{n=0}^{\infty} \frac{\partial}{\partial t}\left(\Psi_{n, t}^{(\xi)} f\right)(x), \\
\frac{\partial}{\partial x_{i}}\left(\sum_{n=0}^{\infty} \Psi_{n, t}^{(\xi)} f\right)(x) & =\sum_{n=0}^{\infty} \frac{\partial}{\partial x_{i}}\left(\Psi_{n, t}^{(\xi)} f\right)(x), \\
\frac{\partial^{2}}{\partial x_{i} \partial x_{j}}\left(\sum_{n=0}^{\infty} \Psi_{n, t}^{(\xi)} f\right)(x) & =\sum_{n=0}^{\infty} \frac{\partial^{2}}{\partial x_{i} \partial x_{j}}\left(\Psi_{n, t}^{(\xi)} f\right)(x), \\
\mathcal{L}\left(\sum_{n=0}^{\infty} \Psi_{n, t}^{(\xi)} f\right)(x) & =\sum_{n=0}^{\infty} \mathcal{L}\left(\Psi_{n, t}^{(\xi)} f\right)(x), \\
\mathcal{N}\left(\sum_{n=0}^{\infty} \Psi_{n, t}^{(\xi)} f\right)(x) & =\sum_{n=0}^{\infty} \mathcal{N}\left(\Psi_{n, t}^{(\xi)} f\right)(x) .
\end{aligned}
$$

Proof Fix $f \in C_{0}\left(\mathbb{R}^{d}\right), \xi \in(0,1]$. By Lemma 4.14, we know that $t \rightarrow \frac{\partial}{\partial t}\left(\Psi_{n, t}^{(\xi)} f\right)(x)$ is continuous on $(0, \tau]$ for each fixed $n \in \mathbb{N}, x \in \mathbb{R}^{d}$. Using this, Lemma 4.1, (102) and (69) we get (103).

Let $t \in(0, \tau], n \in \mathbb{N}, i, j \in\{1, \ldots, d\}$. The fact that $\frac{\partial}{\partial x_{i}}\left(\Psi_{n, t}^{(\xi)} f\right)(x)$ is well defined and continuous as a function of $x \in \mathbb{R}^{d}$ follows from (101), Lemmas 3.4, 3.5, Proposition 3.12 and Lemma 3.19. By the above arguments, we also get

$$
\left|\frac{\partial}{\partial x_{i}}\left(\Psi_{n, t}^{(\xi)} f\right)(x)\right| \leq c(\xi) \sup _{s \in(0, t]}\left\|\Psi_{n-1, s}^{(\xi)} f\right\|_{\infty}, \quad n \geq 1 .
$$

Using this, Lemma 3.19 and Lemma 4.1 we arrive at (104). By similar arguments we obtain that $\frac{\partial^{2}}{\partial x_{i} \partial x_{j}}\left(\Psi_{n, t}^{(\xi)} f\right)(x)$ is well defined and continuous as a function of $x \in \mathbb{R}^{d}$ and

$$
\left|\frac{\partial^{2}}{\partial x_{i} \partial x_{j}}\left(\Psi_{n, t}^{(\xi)} f\right)(x)\right| \leq c(\xi) \sup _{s \in(0, t]}\left\|\Psi_{n-1, s}^{(\xi)} f\right\|_{\infty}, \quad n \geq 1 .
$$

Using this and Lemma 4.1 we get (105).

For any $\zeta>0$ we have

$$
\mathcal{L}_{\zeta}\left(\sum_{n=0}^{\infty} \Psi_{n, t}^{(\xi)} f\right)(x)=\sum_{n=0}^{\infty} \mathcal{L}_{\zeta}\left(\Psi_{n, t}^{(\xi)} f\right)(x)
$$

This, Lemma 4.1, (74) and (75) imply (106). The equality (107) is clear. 
Corollary 4.16 For every $f \in C_{0}\left(\mathbb{R}^{d}\right), \xi \in(0,1]$ the function $T_{t}^{(\xi)} f(x)$ belongs to $C^{1}((0, \infty))$ as a function of t and to $C_{0}^{2}\left(\mathbb{R}^{d}\right)$ as a function of $x$.

Proof Fix $f \in C_{0}\left(\mathbb{R}^{d}\right), \xi \in(0,1]$. The fact that $T_{t}^{(\xi)} f(x)$ belongs to $C^{1}((0, \infty))$ as a function of $t$ follows from (103), Lemma 4.14, (102), (69), Lemma 3.16 and Lemma 4.1. From the proof of Lemma 4.15 we know that for each $t>0, n \in \mathbb{N}, i, j \in\{1, \ldots, d\}$ the function $\frac{\partial^{2}}{\partial x_{i} \partial x_{j}}\left(\Psi_{n, t}^{(\xi)} f\right)(x)$ is continuous as a function of $x \in \mathbb{R}^{d}$. The fact that $T_{t}^{(\xi)} f(x)$ belongs to $C_{0}^{2}\left(\mathbb{R}^{d}\right)$ as a function of $x$ follows from (104), (105), (108), Lemmas 4.1 and 4.10.

Heuristically, now our aim is to show that if $\xi$ is small then $\frac{\partial}{\partial t}\left(T_{t}^{(\xi)} f\right)(x)-\mathcal{K}\left(T_{t}^{(\xi)} f\right)(x)$ is small. For any $t>0, \xi \in(0,1], x \in \mathbb{R}^{d}$ and $f \in C_{0}\left(\mathbb{R}^{d}\right)$ let us denote

$$
\begin{gathered}
\Upsilon_{t}^{(\xi)} f(x)=\frac{\partial}{\partial t}\left(T_{t}^{(\xi)} f\right)(x)-\mathcal{K}\left(T_{t}^{(\xi)} f\right)(x), \\
\Upsilon_{t}^{(\xi, 1)} f(x)=e^{-\lambda t} \sum_{n=1}^{\infty}\left[\int_{\mathbb{R}^{d}} p_{z}(\xi, x-z) \mathcal{N}\left(\Psi_{n-1, t}^{(\xi)} f\right)(z) d z-\mathcal{N}\left(\Psi_{n-1, t}^{(\xi)} f\right)(x)\right], \\
\Upsilon_{t}^{(\xi, 2)} f(x)=e^{-\lambda t} \sum_{n=1}^{\infty} \int_{0}^{t} \Lambda_{t-s}^{(\xi)}\left(\mathcal{N}\left(\Psi_{n-1, s}^{(\xi)} f\right)\right)(x) d s .
\end{gathered}
$$

By Lemma 4.1, (80), (81) and the boundedness of $\mathcal{N}: L^{\infty}\left(\mathbb{R}^{d}\right) \rightarrow L^{\infty}\left(\mathbb{R}^{d}\right)$, the above series are convergent.

Lemma 4.17 For any $t>0, \xi \in(0,1], x \in \mathbb{R}^{d}$ and $f \in C_{0}\left(\mathbb{R}^{d}\right)$ we have

$$
\Upsilon_{t}^{(\xi)} f(x)=e^{-\lambda t} \Lambda_{t}^{(\xi)} f(x)+\Upsilon_{t}^{(\xi, 1)} f(x)+\Upsilon_{t}^{(\xi, 2)} f(x) .
$$

Proof By Lemmas 3.21, 4.14 and 4.15, we get

$$
\begin{aligned}
& \frac{\partial}{\partial t}\left(T_{t}^{(\xi)} f\right)(x)=-\lambda e^{-\lambda t} \Psi_{0, t}^{(\xi)} f(x)+e^{-\lambda t} \mathcal{L}\left(\Psi_{0, t}^{(\xi)} f\right)(x)+e^{-\lambda t} \Lambda_{t}^{(\xi)} f(x) \\
& -\lambda e^{-\lambda t} \sum_{n=1}^{\infty} \Psi_{n, t}^{(\xi)} f(x)+e^{-\lambda t} \sum_{n=1}^{\infty} \mathcal{L}\left(\Psi_{n, t}^{(\xi)} f\right)(x) \\
& \quad+e^{-\lambda t} \sum_{n=1}^{\infty} \mathcal{N}\left(\Psi_{n-1, t}^{(\xi)} f\right)(x)+\Upsilon_{t}^{(\xi, 1)} f(x)+\Upsilon_{t}^{(\xi, 2)} f(x) .
\end{aligned}
$$

Again, by Lemma 4.15 , this is equal to

$$
\begin{aligned}
& \mathcal{L}\left(e^{-\lambda t} \sum_{n=0}^{\infty} \Psi_{n, t}^{(\xi)} f\right)(x)-\lambda\left(e^{-\lambda t} \sum_{n=0}^{\infty} \Psi_{n, t}^{(\xi)} f(x)\right) \\
& \quad+\mathcal{N}\left(e^{-\lambda t} \sum_{n=0}^{\infty} \Psi_{n, t}^{(\xi)} f\right)(x)+e^{-\lambda t} \Lambda_{t}^{(\xi)} f(x)+\Upsilon_{t}^{(\xi, 1)} f(x)+\Upsilon_{t}^{(\xi, 2)} f(x) .
\end{aligned}
$$

Using the definition of $T_{t}^{(\xi)} f$ and noting that $\mathcal{N} g(x)-\lambda g(x)=\mathcal{R} g(x)$ and $\mathcal{L} g(x)+$ $\mathcal{R} g(x)=\mathcal{K} g(x)$ we obtain the assertion of the lemma. 
Lemma 4.18 (i) For any $f \in C_{0}\left(\mathbb{R}^{d}\right)$ we have

$$
\Upsilon_{t}^{(\xi)} f(x) \rightarrow 0, \quad \text { as } \xi \rightarrow 0^{+},
$$

uniformly in $(t, x) \in\left[\tau_{1}, \tau\right] \times \mathbb{R}^{d}$ for every $\tau_{1} \in(0, \tau)$.

(ii) For any $f \in C_{0}\left(\mathbb{R}^{d}\right)$ we have

$$
\int_{0}^{t} \Upsilon_{s}^{(\xi)} f(x) d s \rightarrow 0, \quad \text { as } \quad \xi \rightarrow 0^{+},
$$

uniformly in $(t, x) \in(0, \tau] \times \mathbb{R}^{d}$.

Proof The lemma follows from Lemma 3.22, Proposition 3.10, Lemmas 4.10, 4.1, (80), (81) and the boundedness of $\mathcal{N}: L^{\infty}\left(\mathbb{R}^{d}\right) \rightarrow L^{\infty}\left(\mathbb{R}^{d}\right)$.

The next result (positive maximum principle) is based on the ideas from [19, Section 4.2]. Its proof is very similar to the proof of [19, Lemma 4.3] and it is omitted.

Lemma 4.19 Let us consider the function $v:[0, \infty) \times \mathbb{R}^{d} \rightarrow \mathbb{R}$ and the family of functions $v^{(\xi)}:[0, \infty) \times \mathbb{R}^{d} \rightarrow \mathbb{R}, \xi \in(0,1]$. Assume that for each $\xi \in(0,1]$ $\sup _{t \in(0, \tau], x \in \mathbb{R}^{d}}\left|v^{(\xi)}(x, t)\right|<\infty, v^{(\xi)}$ is $C^{1}$ in the first variable and $C^{2}$ in the second variable. We also assume that (for any $\tau>0$ )

(i)

$$
v^{(\xi)}(t, x) \rightarrow v(t, x) \quad \text { as } \quad \xi \rightarrow 0^{+},
$$

uniformly in $t \in[0, \tau], x \in \mathbb{R}^{d}$;

(ii)

$$
v^{(\xi)}(t, x) \rightarrow 0 \quad \text { as } \quad|x| \rightarrow \infty,
$$

uniformly in $t \in[0, \tau], \xi \in(0,1]$;

(iii) for any $0<\tau_{1}<\tau_{2} \leq \tau$

$$
\frac{\partial}{\partial t} v^{(\xi)}(t, x)-\mathcal{K} v^{(\xi)}(t, x) \rightarrow 0 \quad \text { as } \quad \xi \rightarrow 0^{+},
$$

uniformly in $t \in\left[\tau_{1}, \tau_{2}\right], x \in \mathbb{R}^{d}$;

(iv)

$$
v^{(\xi)}(t, x) \rightarrow v(0, x) \quad \text { as } \quad\left(\xi \rightarrow 0^{+} \text {and } t \rightarrow 0^{+}\right),
$$

uniformly in $x \in \mathbb{R}^{d}$;

(v) for any $x \in \mathbb{R}^{d} v(0, x) \geq 0$.

Then for any $t \geq 0, x \in \mathbb{R}^{d}$ we have $v(t, x) \geq 0$.

Proposition 4.20 $T_{t}: \mathcal{B}_{b}\left(\mathbb{R}^{d}\right) \rightarrow \mathcal{B}_{b}\left(\mathbb{R}^{d}\right)$ is a linear, bounded operator for any $t \in(0, \tau]$. For each $t \in(0, \tau], f \in \mathcal{B}_{b}\left(\mathbb{R}^{d}\right)$ and $R \geq 1$ there exists a sequence $f_{k} \in C_{0}\left(\mathbb{R}^{d}\right), k \in \mathbb{N}$ such that $\lim _{k \rightarrow \infty} f_{k}(x)=f(x)$ for almost all $x \in B(0, R)$; for any $k \in \mathbb{N}$ we have $\left\|f_{k}\right\|_{\infty} \leq\|f\|_{\infty}$ and for any $x \in B(0, R)$ we have $\lim _{k \rightarrow \infty} T_{t} f_{k}(x)=T_{t} f(x)$.

Proof Fix $t \in(0, \tau]$. The fact that $T_{t}: \mathcal{B}_{b}\left(\mathbb{R}^{d}\right) \rightarrow \mathcal{B}_{b}\left(\mathbb{R}^{d}\right)$ is a linear, bounded operator follows by the definition of $T_{t}$ and Lemma 4.1.

Fix $f \in \mathcal{B}_{b}\left(\mathbb{R}^{d}\right), R \geq 1$ and $k \in \mathbb{N}, k \geq 1$. By Lemma 4.10 , there exists $R_{k} \geq R$ such that for any $x \in B(0, R)$ we have

$$
\left|T_{t}\left(f \mathbf{1}_{B^{c}\left(0, R_{k}\right)}\right)(x)\right| \leq \frac{1}{k} .
$$


Put $g_{1, k}(x)=\mathbf{1}_{B\left(0, R_{k}\right)}(x) f(x), g_{2, k}(x)=\mathbf{1}_{B^{c}\left(0, R_{k}\right)}(x) f(x)$. By standard methods, there exists $f_{k} \in C_{0}\left(\mathbb{R}^{d}\right)$ such that

$$
\left\|f_{k}-g_{1, k}\right\|_{1} \leq \frac{1}{k}
$$

and $\operatorname{supp}\left(f_{k}\right) \subset B\left(0, R_{k}+1\right),\left\|f_{k}\right\|_{\infty} \leq\|f\|_{\infty}$. By Theorem 4.3, for any $x \in \mathbb{R}^{d}$, we have

$$
\left|T_{t}\left(f_{k}-g_{1, k}\right)(x)\right| \leq \frac{c\|f\|_{\infty}^{1-\alpha /(2 d)}}{k^{\alpha /(2 d)} t^{1 / 2}} \text {. }
$$

This and (110) imply that for any $x \in B(0, R)$ we have $\lim _{k \rightarrow \infty} T_{t} f_{k}(x)=T_{t} f(x)$. We also have $\left\|f_{k} \mathbf{1}_{B(0, R)}-f \mathbf{1}_{B(0, R)}\right\|_{1} \leq 1 / k$. Hence, there exists a subsequence $k_{m}$ such that $\lim _{m \rightarrow \infty} f_{k_{m}}(x)=f(x)$ for almost all $x \in B(0, R)$.

Proposition 4.21 For any $t \in(0, \infty), x \in \mathbb{R}^{d}$ and $f \in C_{b}^{2}\left(\mathbb{R}^{d}\right)$ we have

$$
T_{t} f(x)=f(x)+\int_{0}^{t} T_{s}(\mathcal{K} f)(x) d s .
$$

Proof Step 1. $f \in C_{0}^{2}\left(\mathbb{R}^{d}\right)$.

For any $t \geq 0, x \in \mathbb{R}^{d}, \xi \in(0,1]$ put

$$
\begin{aligned}
v(t, x) & =T_{t} f(x)-f(x)-\int_{0}^{t} T_{s}(\mathcal{K} f)(x) d s, \\
v^{(\xi)}(t, x) & =T_{t}^{(\xi)} f(x)-f(x)-\int_{0}^{t} T_{s}^{(\xi)}(\mathcal{K} f)(x) d s .
\end{aligned}
$$

Note that $\mathcal{K} f \in C_{0}\left(\mathbb{R}^{d}\right)$. By Lemmas 4.12, 4.18 and Corollary 4.16, we obtain that $v(t, x)$, $v^{(\xi)}(t, x)$ satisfy the assumptions of Lemma 4.19. Note that $v(0, x)=0$ for all $x \in \mathbb{R}^{d}$. The assertion of the proposition for $f \in C_{0}^{2}\left(\mathbb{R}^{d}\right)$ follows from Lemma 4.19.

Step 2. $f \in C_{b}^{2}\left(\mathbb{R}^{d}\right)$.

By standard methods, there exists a sequence $f_{n} \in C_{0}^{2}\left(\mathbb{R}^{d}\right), n=1,2, \ldots$ such that

$$
\sup _{n \in \mathbb{N}} \max _{i, j \in\{1, \ldots, d\}} \sup _{x \in \mathbb{R}^{d}}\left(\left|f_{n}(x)\right|+\left|\frac{\partial f_{n}}{\partial x_{i}}(x)\right|+\left|\frac{\partial^{2} f_{n}}{\partial x_{i} \partial x_{j}}(x)\right|\right)<\infty .
$$

and for any $r \geq 1$ we have $\lim _{n \rightarrow \infty}\left(\sup _{|x| \leq r}\left|f_{n}(x)-f(x)\right|\right)=0$. It follows that $\sup _{n \geq 1, x \in \mathbb{R}^{d}}\left|\mathcal{K} f_{n}(x)\right|<\infty$ and for each $x \in \mathbb{R}^{d}$ we have $\mathcal{K} f_{n}(x) \rightarrow \mathcal{K} f(x)$. By Corollary 4.11, it follows that for each $x \in \mathbb{R}^{d}, t>0$ and $s \in(0, t]$ we have $T_{t} f_{n}(x) \rightarrow T_{t} f(x)$ and $T_{s}\left(\mathcal{K} f_{n}\right)(x) \rightarrow T_{s}(\mathcal{K} f)(x)$. By Step 1 and the dominated convergence theorem, we obtain the assertion of the proposition.

The following result shows that $\left\{T_{t}\right\}$ is a Feller semigroup.

\section{Theorem 4.22 We have}

(i) $T_{t}: C_{0}\left(\mathbb{R}^{d}\right) \rightarrow C_{0}\left(\mathbb{R}^{d}\right)$ for any $t \in(0, \infty)$,

(ii) $T_{t} f(x) \geq 0$ for any $t>0, x \in \mathbb{R}^{d}$ and $f \in C_{0}\left(\mathbb{R}^{d}\right)$ such that $f(x) \geq 0$ for all $x \in \mathbb{R}^{d}$,

(iii) $T_{t} 1_{\mathbb{R}^{d}}(x)=1$ for any $t>0, x \in \mathbb{R}^{d}$,

(iv) $T_{t+s} f(x)=T_{t}\left(T_{s} f\right)(x)$ for any $s, t>0, x \in \mathbb{R}^{d}, f \in C_{0}\left(\mathbb{R}^{d}\right)$,

(v) $\lim _{t \rightarrow 0^{+}}\left\|T_{t} f-f\right\|_{\infty}=0$ for any $f \in C_{0}\left(\mathbb{R}^{d}\right)$. 
(vi) there exists a nonnegative function $p(t, x, y)$ in $(t, x, y) \in(0, \infty) \times \mathbb{R}^{d} \times \mathbb{R}^{d}$; for each fixed $t>0, x \in \mathbb{R}^{d}$ the function $y \rightarrow p(t, x, y)$ is Lebesgue measurable, $\int_{\mathbb{R}^{d}} p(t, x, y) d y=1$ and $T_{t} f(x)=\int_{\mathbb{R}^{d}} p(t, x, y) f(y) d y$ for $f \in C_{0}\left(\mathbb{R}^{d}\right)$.

Proof (i) This follows from Theorem 4.4 and Lemma 4.12 (ii).

(ii) Let $f \in C_{0}\left(\mathbb{R}^{d}\right)$ be such that $f(x) \geq 0$ for all $x \in \mathbb{R}^{d}$. For $t \geq 0, x \in \mathbb{R}^{d}, \xi \in(0,1]$ put $v(t, x)=T_{t} f(x), v^{(\xi)}(t, x)=T_{t}^{(\xi)} f(x)$. By Lemmas 4.12, 4.18 and Corollary 4.16, we obtain that $v(t, x), v^{(\xi)}(t, x)$ satisfy the assumptions of Lemma 4.19. The assertion of Theorem 4.22 (ii) follows from Lemma 4.19.

(iii) The proof is very similar to the proof of [19, Lemma 4.5 b]. Let $f \in C_{0}^{2}\left(\mathbb{R}^{2}\right)$ be such that $f \equiv 1$ on $B(0,1) \subset \mathbb{R}^{d}$ and let $f_{n}(x)=f(x / n), x \in \mathbb{R}^{d}, n \in \mathbb{N}, n \geq 1$. For any $x \in \mathbb{R}^{d}$ we have $\lim _{n \rightarrow \infty} f_{n}(x)=1, \lim _{n \rightarrow \infty} \mathcal{K} f_{n}(x)=0$ and $\sup _{n \in \mathbb{N}, n \geq 1}\left(\left\|f_{n}\right\|_{\infty} \mathrm{V}\right.$ $\left.\left\|\mathcal{K} f_{n}\right\|_{\infty}\right)<\infty$. By Corollary 4.11 , for any $s, t>0$ and $x \in \mathbb{R}^{d}$, we get

$$
\lim _{n \rightarrow \infty} T_{t} f_{n}(x)=T_{t} \mathbf{1}_{\mathbb{R}^{d}}(x), \quad \lim _{n \rightarrow \infty} T_{s}\left(\mathcal{K} f_{n}\right)(x)=0 .
$$

Using (111) for $f_{n}$ and (112) we obtain (iii).

(iv) Let $f \in C_{0}\left(\mathbb{R}^{d}\right)$. For $s, t \geq 0, x \in \mathbb{R}^{d}, \xi \in(0,1]$ put $v(t, x)=T_{t+s} f(x)-$ $T_{t}\left(T_{s} f\right)(x), v^{(\xi)}(t, x)=T_{t+s}^{(\xi)} f(x)-T_{t}^{(\xi)}\left(T_{s} f\right)(x)$. By Lemmas 4.12, 4.18 and Corollary 4.16, we obtain that $v(t, x), v^{(\xi)}(t, x)$ satisfy the assumptions of Lemma 4.19. Note that $v(0, x)=0$ for all $x \in \mathbb{R}^{d}$. The assertion of Theorem 4.22 (iv) follows from Lemma 4.19.

(v) Choose $\varepsilon_{1}>0$. Since $f \in C_{0}\left(\mathbb{R}^{d}\right)$ there exists $\delta_{1}>0$ such that

$$
\forall x, y \in \mathbb{R}^{d} \quad|x-y|<\delta_{1} \Rightarrow|f(x)-f(y)|<\varepsilon_{1} .
$$

Fix arbitrary $x \in \mathbb{R}^{d}, t \in(0, \tau]$. Put $f_{1}(y)=\mathbf{1}_{B\left(x, \delta_{1}\right)}(y)(f(y)-f(x)), f_{2}(y)=$ $\mathbf{1}_{B^{c}\left(x, \delta_{1}\right)}(y)(f(y)-f(x)), y \in \mathbb{R}^{d}$. By (iii), we have

$$
T_{t} f(x)-f(x)=T_{t} f_{1}(x)+T_{t} f_{2}(x) .
$$

We also have

$$
\begin{gathered}
\left|T_{t} f_{1}(x)\right|<c \varepsilon_{1}, \\
\left|T_{t} f_{2}(x)\right| \leq 2\|f\|_{\infty} T_{t} \mathbf{1}_{B^{c}\left(x, \delta_{1}\right)}(x)
\end{gathered}
$$

and

$$
\begin{aligned}
T_{t} \mathbf{1}_{B^{c}\left(x, \delta_{1}\right)}(x)= & e^{-\lambda t} \int_{B^{c}\left(x, \delta_{1}\right)} p_{y}(t, x-y) d y+e^{-\lambda t} \Phi_{t} \mathbf{1}_{B^{c}\left(x, \delta_{1}\right)}(x) \\
& +e^{-\lambda t} \sum_{n=1}^{\infty} \Psi_{n, t} \mathbf{1}_{B^{c}\left(x, \delta_{1}\right)}(x) .
\end{aligned}
$$

By Proposition 3.10, there exists $\tau_{1} \in(0, \tau]$ such that

$$
\forall t \in\left(0, \tau_{1}\right] \quad \int_{B^{c}\left(x, \delta_{1}\right)} p_{y}(t, x-y) d y<\varepsilon_{1} .
$$

By Proposition 3.12, we obtain that

$$
\forall t \in\left(0, \tau_{1}\right] \quad\left|\Phi_{t} \mathbf{1}_{B^{c}\left(x, \delta_{1}\right)}(x)\right| \leq c \tau_{1}^{1 / 2} .
$$


By Lemma 4.1, we obtain that

$$
\forall t \in\left(0, \tau_{1}\right] \quad\left|e^{-\lambda t} \sum_{n=1}^{\infty} \Psi_{n, t} \mathbf{1}_{B^{c}\left(x, \delta_{1}\right)}(x)\right| \leq c t .
$$

This implies (v).

(vi) This follows from (i), (ii), (iii) and Theorem 4.3.

We are now in a position to provide the proof of Theorems 1.1 and 1.2.

Proof of Theorem 1.1 From Theorem 4.22 we conclude that there is a Feller process $\tilde{X}_{t}$ with the semigroup $T_{t}$ on $C_{0}\left(\mathbb{R}^{d}\right)$. Let $\tilde{\mathbb{P}}^{x}, \tilde{\mathbb{E}}^{x}$ be the distribution and expectation of the process $\tilde{X}_{t}$ starting from $x \in \mathbb{R}^{d}$.

By Theorem 4.22 (vi), Proposition 4.20 and Lemma 4.10, we get

$$
\tilde{\mathbb{E}}^{x} f\left(\tilde{X}_{t}\right)=T_{t} f(x)=\int_{\mathbb{R}^{d}} p(t, x, y) f(y) d y \quad f \in \mathcal{B}_{b}\left(\mathbb{R}^{d}\right), t>0, x \in \mathbb{R}^{d} .
$$

By Proposition 4.21 , for any function $f \in C_{b}^{2}\left(\mathbb{R}^{d}\right)$, the process

$$
M_{t}^{\tilde{X}, f}=f\left(\tilde{X}_{t}\right)-f\left(\tilde{X}_{0}\right)-\int_{0}^{t} \mathcal{K} f\left(\tilde{X}_{s}\right) d s
$$

is a $\left(\tilde{\mathbb{P}}^{x}, \mathcal{F}_{t}\right)$ martingale, where $\mathcal{F}_{t}$ is a natural filtration. That is $\tilde{\mathbb{P}}^{x}$ solves the martingale problem for $\left(\mathcal{K}, C_{b}^{2}\left(\mathbb{R}^{d}\right)\right)$. On the other hand, according to [1, Theorem 6.3], the unique solution $X$ to the stochastic equation (1) has the law which is the unique solution to the martingale problem for $\left(\mathcal{K}, C_{b}^{2}\left(\mathbb{R}^{d}\right)\right)$. Hence $\tilde{X}$ and $X$ have the same law so for any $t>0$, $x \in \mathbb{R}^{d}$ and any Borel bounded set $A \subset \mathbb{R}^{d}$ we have

$$
\sigma_{t}(x, A)=\int_{A} p(t, x, y) d y,
$$

where $\sigma_{t}(x, A)$ is defined by (14). Using this, (15) and (113) we obtain

$$
P_{t} f(x)=T_{t} f(x), \quad t>0, x \in \mathbb{R}^{d}, f \in \mathcal{B}_{b}\left(\mathbb{R}^{d}\right) .
$$

Now the assertion of Theorem 1.1 follows from Theorem 4.4 and (114).

Proof of Theorem 1.2 The result follows from Theorem 4.3 and (114).

Remark 4.23 One of the referees suggested the following elegant approach to the strong Feller property, which combines the Feller property with the results obtained in [10]. By the the main theorem of [10] or rather its proof, the transition density $p(t, x, \cdot)$ of the solution to the equation (1) exists for every $x$ and it belongs to the classical Besov space $B_{1, \infty}^{s}\left(\mathbb{R}^{d}\right)$ for some $0<s<1$, and

$$
\sup _{x \in \mathbb{R}^{d}}\|p(t, x, \cdot)\|_{B_{1, \infty}^{s}}<\infty
$$

where $\|\cdot\|_{B_{1, \infty}^{s}}$ is a Besov norm in $B_{1, \infty}^{s}\left(\mathbb{R}^{d}\right)$. There is a classical but very deep result in the theory of function spaces which states that the Besov space $B_{1, \infty}^{s}\left(\mathbb{R}^{d}\right)$ is compactly embedded into $L^{1}(\Omega)$, where $\Omega \subset \mathbb{R}^{d}$ is an arbitrary nonempty bounded open set. This means that for any sequence $f_{n} \in B_{1, \infty}^{s}\left(\mathbb{R}^{d}\right)$ such that $\sup _{n}\left\|f_{n}\right\|_{B_{1, \infty}^{s}}<\infty$ the sequence $f_{n} \mathbf{1}_{\Omega}$ is relatively compact in $L^{1}(\Omega)$.

Let $x_{n}, n \geq 1$, be such that $\lim _{n \rightarrow \infty} x_{n}=x_{0}, f$ be a bounded Borel function and $\epsilon>0$. Since $A(\cdot)$ is Lipschitz continuous, the semigroup determined by the solution of to the 
equation (1) has the Feller property, see eg. [33]. This implies that the sequence of measures $m_{n}(d y)=p\left(t, x_{n}, y\right) d y$ is weakly convergent to $m_{0}(d y)=p\left(t, x_{0}, y\right) d y$. Hence the set of the truncated measures $\mathbf{1}_{\Omega} m_{n}(d y)$ is weakly convergent to $\mathbf{1}_{\Omega} m_{0}(d y)$ provided that $\partial \Omega$ has the Lebesgue measure 0 . By compact embedding any subsequence $\left\{x_{k_{n}}\right\}$ has a subsequence $\left\{x_{l_{n}}\right\}$ such that $p\left(t, x_{l_{n}}, \cdot\right) \mathbf{1}_{\Omega}$ is convergent to $q(\cdot)$ in $L^{1}(\Omega)$. Due to the weak convergence of the truncated measures we have that $q(\cdot)=p\left(t, x_{0}, \cdot\right)$ in $L^{1}(\Omega)$. This implies

$$
\begin{aligned}
& \limsup _{n \rightarrow \infty}\left|\int f(y) p_{t}\left(x_{n}, y\right) d y-\int f(y) p_{t}\left(x_{0}, y\right) d y\right| \\
\leq & \limsup _{n \rightarrow \infty}\|f\|_{\infty}\left(\left\|p_{t}\left(x_{n}, \cdot\right)-p_{t}\left(x_{0}, \cdot\right)\right\|_{L^{1}(\Omega)}+2 \sup _{n \geq 0} m_{n}\left(\Omega^{c}\right)\right) \\
= & \limsup _{n \rightarrow \infty} 2\|f\|_{\infty} \sup _{n \geq 0} m_{n}\left(\Omega^{c}\right) \\
\leq & \epsilon,
\end{aligned}
$$

provided $\Omega$ is large enough. Indeed, $\sup _{n>0} m_{n}\left(\Omega^{c}\right)$ can be made arbitrarily small by the tightness of $\left\{m_{n} ; n \geq 0\right\}$, which follows from some moment estimates on $X_{t}$. This shows that the above limit must be 0 . It is worth noticing that this argument works in our setup for all $0<\alpha<2$. On the other hand we do not think that from the results of [10] one can obtain our main estimate (6).

Remark 4.24 For any $\alpha \in(0,1), d \geq 2$ there exist $A(x)$ satisfying (2-4) and $t>0$ such that $P_{t}: L^{1}\left(\mathbb{R}^{d}\right) \rightarrow L^{\infty}\left(\mathbb{R}^{d}\right)$ is not bounded. For simplicity we will present an example for $d=2$ but similar examples can be constructed for $d>2$.

Proof First we define $A\left(x_{1}, x_{2}\right)$. Let $\kappa(r):[0, \infty) \rightarrow[0, \infty)$ be defined by $\kappa(r)=0$ for $r \in[0,1], \kappa(r)=r-1$ for $r \in(1,1+\pi / 4], \kappa(r)=\pi / 4$ for $r>1+\pi / 4$. It is easy to check that $\kappa(r)=((r-1) \vee 0) \wedge(\pi / 4)$ and that it is a Lipschitz function. Now let us introduce standard polar coordinates $(r, \varphi), r \in[0, \infty), \varphi \in[0,2 \pi)$ by $x_{1}=r \cos \varphi, x_{2}=r \sin \varphi$.

We put $A\left(x_{1}, x_{2}\right)=\tilde{A}(r, \varphi)=\left[\begin{array}{cc}\cos (\tilde{\theta}(r, \varphi)) & -\sin (\tilde{\theta}(r, \varphi)) \\ \sin (\tilde{\theta}(r, \varphi)) & \cos (\tilde{\theta}(r, \varphi))\end{array}\right]$, where $\tilde{\theta}(r, \varphi)$ is defined in the following way. $\tilde{\theta}(r, \varphi)=0$ for $r \in[0,1], \varphi \in[0,2 \pi), \tilde{\theta}(r, \varphi)=\varphi$ for $r \in(1,1+$ $\pi / 4], \varphi \in[0, \kappa(r)], \tilde{\theta}(r, \varphi)=2 \kappa(r)-\varphi$ for $r \in(1,1+\pi / 4], \varphi \in(\kappa(r), 2 \kappa(r)], \tilde{\theta}(r, \varphi)=0$ for $r \in(1,1+\pi / 4], \varphi \in[2 \kappa(r), 2 \pi), \tilde{\theta}(r, \varphi)=\varphi$ for $r>1+\pi / 4, \varphi \in[0, \pi / 4]$, $\tilde{\theta}(r, \varphi)=\pi / 2-\varphi$ for $r>1+\pi / 4, \varphi \in(\pi / 4, \pi / 2], \tilde{\theta}(r, \varphi)=0$ for $r>1+\pi / 4$, $\varphi \in(\pi / 2,2 \pi)$.

One can check that $A\left(x_{1}, x_{2}\right)=\left[\begin{array}{cc}\cos \left(\theta\left(x_{1}, x_{2}\right)\right) & -\sin \left(\theta\left(x_{1}, x_{2}\right)\right) \\ \sin \left(\theta\left(x_{1}, x_{2}\right)\right) & \cos \left(\theta\left(x_{1}, x_{2}\right)\right)\end{array}\right]$, where $\theta(0,0)=0$ and for $\left(x_{1}, x_{2}\right) \neq(0,0)$

$$
\theta\left(x_{1}, x_{2}\right)=\left(\kappa\left(\sqrt{x_{1}^{2}+x_{2}^{2}}\right)-\left|\left(\left(\operatorname{Arg}\left(x_{1}+i x_{2}\right) \vee 0\right) \wedge \frac{\pi}{2}\right)-\kappa\left(\sqrt{x_{1}^{2}+x_{2}^{2}}\right)\right|\right) \vee 0 .
$$

It is clear that $A(x)$ satisfies (2-4).

Put $D=B((3,1), 1)$. Note that for any $x \in \mathbb{R}^{2}$ such that $x_{2} \in\left[0, x_{1}\right]$ and $|x| \geq \pi / 4+1$ we have $A(x)=A\left(x_{1}, x_{2}\right)=|x|^{-1}\left[\begin{array}{cc}x_{1} & -x_{2} \\ x_{2} & x_{1}\end{array}\right]$. In particular, this holds for $x \in D$. 
For any $f \in \mathcal{B}_{b}\left(\mathbb{R}^{d}\right), t>0, x \in \mathbb{R}^{d}$ we have $P_{t} f(x)=T_{t} f(x)=$ $e^{-\lambda t} \sum_{n=0}^{\infty} \Psi_{n, t} f(x)$. For our purposes it is enough to study $\Psi_{1, t}$. We have

$$
\begin{aligned}
& \Psi_{1, t} f(x)=\int_{0}^{t} U_{t-s}\left(\mathcal{N}\left(U_{s} f\right)\right)(x) d s= \\
& \sum_{i=1}^{2} \int_{0}^{t} \int_{\mathbb{R}^{2}} u(t-s, x, z) \int_{\mathbb{R}} \int_{\mathbb{R}^{2}} u\left(s, z+a_{i}(z) w, y\right) f(y) d y v(w) d w d z d s
\end{aligned}
$$

By arguments similar to the proof of Theorem 4.22, one can show that for any $t>0, x \in \mathbb{R}^{d}$ and almost all $y \in \mathbb{R}^{d}$ we have $u(t, x, y) \geq 0$ and for any $s, t>0, x \in \mathbb{R}^{d}, f \in \mathcal{B}_{b}\left(\mathbb{R}^{d}\right)$ we have $U_{t+s} f(x)=U_{t}\left(U_{s} f\right)(x)$, (we omit the details here). Put

$$
u_{1}(t, x, y)=\sum_{i=1}^{2} \int_{0}^{t} \int_{\mathbb{R}^{2}} u(t-s, x, z) \int_{\mathbb{R}} u\left(s, z+a_{i}(z) w, y\right) v(w) d w d z d s
$$

By (115), we have

$$
\Psi_{1, t} f(x)=\int_{\mathbb{R}^{2}} u_{1}(t, x, y) f(y) d y .
$$

By Lemma 3.23 and the semigroup property of $U_{t}$, one can easily obtain that there exists $t_{2}>1$ such that for any $t \in\left[t_{2}-1, t_{2}\right]$,

$$
\int_{D} u(t, 0, z) d z \geq c
$$

Now our aim will be to estimate from below $u_{1}\left(t_{2}, 0, y\right)$ for $y$ which are sufficiently close to 0 . Let $c_{1}, \varepsilon_{1}, t_{1}$ be the constants from Lemma 3.23. First, note that for $z \in D$ we have $a_{1}(z)=\left(a_{11}(z), a_{21}(z)\right)=|z|^{-1}\left(z_{1}, z_{2}\right)=z /|z|$. Hence $\left|z+a_{1}(z) w\right|=|z|+w$ for any $z \in D, w \in \mathbb{R}$. It follows that for $z \in D$ we have

$$
w \in\left(-|z|-\varepsilon_{1} s^{1 / \alpha} / 2,-|z|+\varepsilon_{1} s^{1 / \alpha} / 2\right) \Longleftrightarrow\left|z+a_{1}(z) w\right|<\varepsilon_{1} s^{1 / \alpha} / 2 .
$$

Therefore, for $z \in D,|y| \leq \varepsilon_{1} s^{1 / \alpha} / 2, w \in\left(-|z|-\varepsilon_{1} s^{1 / \alpha} / 2,-|z|+\varepsilon_{1} s^{1 / \alpha} / 2\right)$, we have $\left|z+a_{i}(z) w-y\right| \leq\left|z+a_{i}(z) w\right|+|y| \leq \varepsilon_{1} s^{1 / \alpha}$. Note also that

$$
|y| \leq \varepsilon_{1} s^{1 / \alpha} / 2 \Longleftrightarrow\left(2|y| / \varepsilon_{1}\right)^{\alpha} \leq s .
$$

Hence, by Lemma 3.23, for $\left(2|y| / \varepsilon_{1}\right)^{\alpha} \leq t_{1} / 2, s \in\left[\left(2|y| / \varepsilon_{1}\right)^{\alpha}, t_{1}\right], z \in D$ we have

$$
\int_{-|z|-\varepsilon_{1} s^{1 / \alpha} / 2}^{-|z|+\varepsilon_{1} s^{1 / \alpha} / 2} u\left(s, z+a_{1}(z) w, y\right) v(w) d w \geq c \varepsilon_{1} s^{1 / \alpha} \frac{c_{1}}{s^{2 / \alpha}}=\frac{c c_{1} \varepsilon_{1}}{s^{1 / \alpha}} .
$$

Recall that $t_{1} \in(0,1]$ and $t_{2}>1 \geq t_{1}$. By (117), for any $s \in\left(0, t_{1}\right]$, we have

$$
\int_{D} u\left(t_{2}-s, 0, z\right) d z \geq c .
$$


Therefore, by (116), nonnegativity of $u(\cdot, \cdot, \cdot),(118),(119)$ for $\left(2|y| / \varepsilon_{1}\right)^{\alpha} \leq t_{1} / 2$ we have

$$
\begin{aligned}
u_{1}\left(t_{2}, 0, y\right) \geq & \int_{\left(2|y| / \varepsilon_{1}\right)^{\alpha}}^{t_{1}} \int_{D} u\left(t_{2}-s, 0, z\right) \int_{-|z|-\varepsilon_{1} s^{1 / \alpha} / 2}^{-|z|+\varepsilon_{1} s^{1 / \alpha} / 2} u\left(s, z+a_{1}(z) w, y\right) \\
& \times v(w) d w d z d s \\
\geq & c \int_{\left(2|y| / \varepsilon_{1}\right)^{\alpha}}^{t_{1}} s^{-1 / \alpha} d s \\
\geq & c|y|^{\alpha-1} .
\end{aligned}
$$

(One can show that in similar examples for $d>2$ we have $u_{1}\left(t_{2}, 0, y\right) \geq c|y|^{\alpha+1-d}$.)

Observe that $\left(2|y| / \varepsilon_{1}\right)^{\alpha} \leq t_{1} / 2 \Longleftrightarrow|y| \leq t_{1}^{1 / \alpha} \varepsilon_{1} 2^{-1-1 / \alpha}$. For $r \in\left(0, t_{1}^{1 / \alpha} \varepsilon_{1} 2^{-1-1 / \alpha}\right)$ we get by (120)

$$
T_{t_{2}} \mathbf{1}_{B(0, r)}(0) \geq e^{-\lambda t_{2}} \Psi_{1, t_{2}} \mathbf{1}_{B(0, r)}(0)=e^{-\lambda t_{2}} \int_{B(0, r)} u_{1}\left(t_{2}, 0, y\right) d y \geq c r^{\alpha+1} .
$$

By (114) we have $T_{t}=P_{t}$. By Theorem $1.1 x \rightarrow P_{t} \mathbf{1}_{B(0, r)}(x)$ is continuous so $\left\|P_{t} \mathbf{1}_{B(0, r)}\right\|_{\infty} \geq P_{t} \mathbf{1}_{B(0, r)}(0)$. Using this and (121) for $r \in\left(0, t_{1}^{1 / \alpha} \varepsilon_{1} 2^{-1-1 / \alpha}\right)$ we get

$$
\frac{\left\|P_{t_{2}} 1_{B(0, r)}\right\|_{\infty}}{\left\|1_{B(0, r)}\right\|_{1}} \geq \frac{P_{t_{2}} \mathbf{1}_{B(0, r)}(0)}{\left\|\mathbf{1}_{B(0, r)}\right\|_{1}} \geq c r^{\alpha-1},
$$

which implies the assertion of the remark. (One can show that in similar examples for $d>2$ we have $\left\|P_{t_{2}} \mathbf{1}_{B(0, r)}\right\|_{\infty} /\left\|\mathbf{1}_{B(0, r)}\right\|_{1} \geq c r^{\alpha+1-d}$.)

Remark 4.25 In the above example (in $\mathbb{R}^{2}$ ) we showed that the transition density $p(t, 0, y)$, for some $t>0$, is an unbounded function. In fact, the following estimate holds $y$ almost surely

$$
p(t, 0, y) \geq c|y|^{\alpha-1}, \quad|y| \leq \varepsilon_{1},
$$

where $c, \varepsilon_{1}$ are some positive constants possibly dependent on $t$.

Hence, we can not expect a general result saying that, with our assumptions, we have the standard estimates for $p(t, x, y)$ of the form

$$
p(t, x, y) \leq C t^{-d / \alpha},
$$

as for example in the case of diagonal matrices [24], or matrices satisfying some further regularity assumptions [31]. On the other hand, the assumption $\alpha<1$ plays an important role (in $\mathbb{R}^{2}$ ), since for $\alpha>1$, by the results of [5], the transition density is bounded.

Remark 4.26 One of the consequences of the results obtained in the paper is the following representation of the transition density

$$
p(t, x, y)=p_{1}(t, x, y)+p_{2}(t, x, y)+p_{3}(t, x, y),
$$

where $p_{1}(t, x, y)=\operatorname{det}(B(y)) G_{t}\left((y-x)(B(y))^{T}\right)$ and $p_{2}(t, x, y)=u(t, x, y)-$ $p_{1}(t, x, y)$. It is obvious that $\int_{\mathbb{R}^{d}} p_{1}(t, x, y) d x=1$. By Proposition 3.10 and Corollary 3.3 we have $\int_{\mathbb{R}^{d}} p_{1}(t, x, y) d y \leq c, p_{1}(t, x, y) \leq c t^{-d / \alpha} e^{-c_{1}|x-y|}, p_{1}(t, x, \cdot)$ weakly converges to $\delta_{x}$ as $t \rightarrow 0^{+}$. By Corollary 3.13 we have $\left|p_{2}(t, x, y)\right| \leq c t^{-d / \alpha} e^{-c_{1} \sqrt{|x-y|}}$. By (25), (57) and (58) we obtain $\int_{\mathbb{R}^{d}}\left|p_{2}(t, x, y)\right| d y \leq c t^{1 / 2}, \int_{\mathbb{R}^{d}}\left|p_{2}(t, x, y)\right| d x \leq c t^{1 / 2}$. By Lemma 4.1 we have $\int_{\mathbb{R}^{d}}\left|p_{3}(t, x, y)\right| d y \leq c t$. 
Acknowledgments We thank prof. J. Zabczyk for communicating to us the problem of the strong Feller property for solutions of SDEs driven by cylindrical $\alpha$-stable processes. We also thank A. Kulik for discussions on the problem treated in the paper. We are very grateful to the referees for valuable comments and remarks which greatly improved the presentation of the paper. In particular we thank one of the referees who offered an alternative approach to the strong Markov property based on the theory of Besov spaces and the results of [10], see Remark 4.23.

Open Access This article is licensed under a Creative Commons Attribution 4.0 International License, which permits use, sharing, adaptation, distribution and reproduction in any medium or format, as long as you give appropriate credit to the original author(s) and the source, provide a link to the Creative Commons licence, and indicate if changes were made. The images or other third party material in this article are included in the article's Creative Commons licence, unless indicated otherwise in a credit line to the material. If material is not included in the article's Creative Commons licence and your intended use is not permitted by statutory regulation or exceeds the permitted use, you will need to obtain permission directly from the copyright holder. To view a copy of this licence, visit http://creativecommonshorg/licenses/by/4.0/.

\section{References}

1. Bass, R., Chen, Z.-Q.: Systems of equations driven by stable processes, Probab. Theory Related Fields 134(2), 175-214 (2006)

2. Blumenthal, R.M., Getoor, R.K.: Some theorems on stable processes. Trans. Amer. Math. Soc. 95, 263273 (1960)

3. Bogdan, K., Grzywny, T., Ryznar M.: Density and tails of unimodal convolution semigroups. J. Funct. Anal. 266, 3543-3571 (2014)

4. Bogdan, K., Jakubowski, T.: Estimates of heat kernel of fractional Laplacian perturbed by gradient operators. Comm. Math. Phys. 271(1), 179-198 (2007)

5. Bogdan, K., Knopova, V., Sztonyk, P.: Heat kernel of anisotropic nonlocal operators. Doc. Math. 25, $1-54$ (2020)

6. Chen, Z.-Q., Kim, P., Song, R.: Dirichlet heat kernel estimates for fractional Laplacian with gradient perturbation. Ann. Probab. 40, 2483-2538 (2012)

7. Chen, Z.-Q., Zhang, X.: Heat kernels and analyticity of non-symmetric jump diffusion semigroups. Probab. Theory Relat. Fields 165(1-2), 267-312 (2016)

8. Chen, Z.-Q., Zhang, X.: Heat kernels for time-dependent non-symmetric stable-like operators. J. Math. Anal. Appl. 465, 1-21 (2018)

9. Clarke, F.H.: On the inverse function theorem. Pac. J. Math. 64(1), 97-102 (1976)

10. Debussche, A., Fournier, N.: Existence of densities for stable-like driven SDE's with Hölder continuous coefficients. J. Funct. Anal. 264(8), 1757-1778 (2013)

11. Dong, Z., Peng, X., Song, Y., Zhang, X.: Strong Feller properties for degenerate SDEs with jumps. Ann. Inst. H. Poincaré, Probab. Statist. 52, 888-897 (2016)

12. Friedman, A.: Partial differential equations of parabolic type, Prentice-Hall, englewood cliffs N.J. (1975)

13. Grzywny, T., Szczypkowski, K.: Heat kernels of non-symmetric lévy-type operators. J. Differential Equations 267(10), 6004-6064 (2019)

14. Hajłasz, P.: Change of variables formula under minimal assumptions. Colloq. Math. 64(1), 93-101 (1993)

15. Jin, P.: Heat kernel estimates for non-symmetric stable-like processes, arXiv:1709.02836 (2017)

16. Kaleta, K., Sztonyk, P.: Estimates of transition densities and their derivatives for jump lévy processes. J. Math. Anal. Appl. 431, 260-282 (2015)

17. Kim, P., Song, R., Vondraček, Z.: Heat kernels of non-symmetric jump processes: beyond the stable case. Potential Anal. 49(1), 37-90 (2018)

18. Knopova, V., Kulik, A.: Intrinsic compound kernel estimates for the transition probability density of lévy-type processes and their applications. Probab. Math. Statist. 37(1), 53-100 (2017)

19. Knopowa, V., Kulik, A.: Parametrix construction of the transition probability density of the solution to an SDE driven by $\alpha$-stable noise. Ann. Inst. H. Poincaré, Probab. Statist. 54, 100-140 (2018)

20. Kochubei, A.N.: Probab pseudodifferential equations, hypersingular integrals and Markov processes Math. USSR Izv. 33 (1989), 233-259; translation from Izv. Akad. Nauk SSSR, Ser. Mat. 52 909-934 (1988) 
21. Kühn, F.: Transition probabilities of lévy-type processes: Parametrix construction. Math. Nachr. 292, 358-376 (2019)

22. Kulczycki, T., Ryznar, M.: Gradient estimates of Dirichlet heat kernels for unimodal lévy processes. Math. Nachr. 291, 374-397 (2018)

23. Kulczycki, T., Ryznar, M.: Gradient estimates of harmonic functions and transition densities for lévy processes. Trans. Amer. Math. Soc. 368(1), 281-318 (2016)

24. Kulczycki, T., Ryznar, M.: Transition density estimates for diagonal systems of SDEs driven by cylindrical $\alpha$-stable processes, ALEA Lat. Am. J. Probab. Math. Stat. 15, 1335-1375 (2018)

25. Kusuoka, S., Marinelli, C.: On smoothing properties of transition semigroups associated to a class of SDEs with jumps. Ann. Inst. H. Poincaré, Probab. Statist. 50, 1347-1370 (2014)

26. Ladyzenskaja, O.A., Solonnikov, V.A., Ural'ceva, N.N.: Linear and Quasi-linear Equations of Parabolic Type (Translated from the Russian by S.Smith). American Mathematical Society, Providence (1968)

27. Liang, M., Wang, J.: Gradient Estimates and Ergodicity for SDEs Driven by Multiplicative Lévy Noises via Coupling Stochastic Process. Appl. https://doi.org/10.1016/j.spa.2019.09.001

28. Liang, M., Wang, J.: Spatial regularity of semigroups generated by Lévy type operators. Math. Nachr. 292 (2019)

29. Levi, E.E.: Sulle equazioni lineari totalmente ellittiche alle derivate parziali. Rend. Circ. Mat. Palermo 24, 275-317 (1907)

30. Métivier, M.: Semimartingales. A Course on Stochastic Processes. Walter de Gruyter, Berlin (1982)

31. Picard, J.: Density in small time at accessible points for jump processes. Stochastic Process Appl. 67(2), 251-279 (1997)

32. Priola, E., Zabczyk, J.: Structural properties of semilinear SPDEs driven by cylindrical stable processes. Probab. Theory Related Fields 149(1-2), 97-137 (2011)

33. Schilling, R., Schnurr, A.: The symbol associated with the solution of a stochastic differential equation. Electron J. Probab. 15, 1369-1393 (2010)

34. Schilling, R., Sztonyk, P., Wang, J.: Coupling property and gradient estimates for lévy processes via the symbol. Bernoulli 18, 1128-1149 (2012)

35. Sztonyk, P.: Estimates of densities for lévy processes with lower intensity of large jumps. Math. Nachr. 290(1), 120-141 (2017)

36. Takeuchi, A.: The Bismut-Elworthy-Li-type formulae for stochastic differential equations with jumps. J. Theoret. Probab. 23, 576-604 (2010)

37. Wang, F.-Y.: Gradient estimate for Ornstein-Uhlenbeck jump processes. Stochastic Process Appl. 121(3), 466-478 (2011)

38. Wang, F.-Y., Xu, L., Zhang, X.: Gradient estimates for SDEs driven by multiplicative lévy noise. J. Funct. Anal. 269, 3195-3219 (2015)

39. Wang, L., Zhang, X.: Harnack Inequalities for SDEs Driven by Cylindrical $\alpha$-stable Processes. Potential Anal. 42(3), 657-669 (2015)

40. Xie, L., Zhang, X.: Ergodicity of stochastic differential equations with jumps and singular coefficients, Ann. Inst. H. Poincare Probab. Statist. (to appear)

41. Xie, L., Zhang, X.: Heatkernelestimates forcriticalfractionaldiffusion. Studia Math. 224(3), 221-263 (2014)

42. Zhang, X., formulas, D.erivative.: Gradient estimates for SDEs driven by $\alpha$-stable processes Stochastic Process. Appl. 123, 1213-1228 (2013)

Publisher's Note Springer Nature remains neutral with regard to jurisdictional claims in published maps and institutional affiliations. 